\title{
College and University Library Statistics, I953־54
}

Fewer libraries return the ACRL

Group I Statistics Questionnaire each year. Eighty institutions sent in figures for $1953 / 54$ whereas 100 libraries replied in 1949/50. The continuity of reporting by the same institutions is increasing, and this year 64 libraries included in last year's compilation are repeated. Three libraries included in the tabulation a year ago failed to submit reports this year.

Only those institutions are included which report some salary data. This year more libraries submitted this important information although 17 libraries still withheld it for the top administrative positions.

Most librarians indicated that documents were included in the "volumes added" figure only if they were bound and fully cataloged. Only one library indicated a use of the bibliographical method of counting holdings, while two others reported a modified system of both physical and bibliographical methods. A few librarians did not answer the question.

Twenty-one, or $30 \%$ of the libraries reporting included serials in the periodical figure. There is apparent difficulty in sorting periodical titles out of a serials records file.

Although the questionnaire stipulated that only students enrolled in the Graduate College be reported as graduate students many libraries obviously included students in professional schools in this figure. Likewise some enrollment figures included extension students while others excluded them. If the statistics are to have any value for comparative purposes, consistency of reporting these statistics is essential.

Additional information was added this year on the length of the work week. Even though the trend seems to be toward a 40 hour 5 day week, it should be pointed out that many public service departments work a $5^{\frac{1}{2}}$ day week. If the non-professional employee's work week differed from the professional, the data for the professional staff were reported. (D.M.B.)

\section{One hundred sixty-two Groups II and III libraries in Groups II and III reported this} year compared with 140 last year, 158 the year before and 142 three years ago. Seventy of the 86 reports received in Group II and 70 of the 76 received in Group III were used in this compilation. Libraries with total operating expenses of $\$ 33,000$ or more are included in Group II. Libraries operating with less than that figure are included in Group III.

Medians were determined from the figures appearing without footnotes. Footnotes were omitted wherever possible to permit more numbers to be used in the calculation of the medians. (D.G.)

\section{Teacher College Libraries}

Again this year not enough questionnaires were returned by libraries in this group to fill the allotted space. The chairman of the committee made a special effort to increase the number to at least the maximum that could be used, but his efforts failed. On October II, after the deadline for receipt of completed questionnaires had passed, he sent a reminder to those who had not returned them urging that they be sent in. A few replies that he received to these requests indicate that some librarians feel that they are doing the committee a favor to send in the questionnaire reports. These statistical tables are certainly not compiled for the pleasure of members of the committee. Many expressions over the years have shown that the statistics are very useful to many library administrators. It is to be regretted that there is still a lack of interest on the part of many, however, to the extent that they won't bother to report information about their own libraries. This year's reports fall short of the highest number that has been reported in the past, but represents four more than reported last year. All questionnaires that were received up to November 2 are included in this tabulation. (W.W.S.) 
Library Operating

\begin{tabular}{|c|c|c|c|c|c|c|c|c|c|c|}
\hline Library 1 & $\begin{array}{l}\text { Fiscal } \\
\text { Year } \\
\text { Ending }\end{array}$ & $\begin{array}{l}\text { Student E } \\
\text { Total } \\
\text { Under- } \\
\text { graduate }\end{array}$ & $\begin{array}{l}\text { rollment² } \\
\text { Total } \\
\text { Gradu- } \\
\text { ate }\end{array}$ & $\begin{array}{l}\text { Book } \\
\text { Stock }\end{array}$ & $\begin{array}{l}\text { Volumes } \\
\text { Added }\end{array}$ & $\begin{array}{l}\text { News- } \\
\text { papers }\end{array}$ & $\begin{array}{l}\text { Periodi- } \\
\text { cals }\end{array}$ & $\begin{array}{c}\text { Staff } \\
\text { Salaries }\end{array}$ & $\begin{array}{c}\text { Student } \\
\text { Service }\end{array}$ & $\begin{array}{l}\text { Staff } \\
\text { Salaries and } \\
\text { Student } \\
\text { Service }\end{array}$ \\
\hline 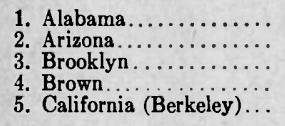 & $\begin{array}{l}\text { 30Se54 } \\
\text { 30Je54 } \\
\text { 30Je54 } \\
\text { 30Je54 } \\
\text { 30Je54 }\end{array}$ & $\begin{array}{r}5,249 \\
4,969 \\
14,834 \\
2,722 \\
11,503\end{array}$ & $\begin{array}{r}403 \\
345 \\
1,242 \\
368 \\
3,824\end{array}$ & $\begin{array}{c}470,748 \\
255,118 \\
242,924 \\
817,514 \\
1,986,81816\end{array}$ & $\begin{array}{r}31,891 \\
9,032 \\
12,582 \\
20,363 \\
79,279\end{array}$ & $\begin{array}{r}109 \\
45 \\
8 \\
20 \\
250\end{array}$ & $\begin{array}{c}3,173 \\
3,151^{18} \\
824 \\
6,000^{18} \\
23,177^{18}\end{array}$ & $\begin{array}{r}161,974 \\
70,200 \\
146,553 \\
150,749 \\
1,192,004\end{array}$ & $\begin{array}{r}25,631 \\
21,079 \\
45,099 \\
16,055 \\
241,027\end{array}$ & $\begin{array}{r}187,605 \\
91,279 \\
191,652 \\
166,804 \\
1,438,031\end{array}$ \\
\hline $\begin{array}{l}\text { 6. California (Lo3 Angeles) } \\
\text { 7. Catholic U. of America. } \\
\text { 8. Cincinnati. } \\
\text { 9. City College (N.Y.)..... } \\
\text { 10. Colorado } \ldots \ldots \ldots \ldots\end{array}$ & $\begin{array}{c}\text { 30Je54 } \\
\text { 30Je54 } \\
\text { 31Ag54 } \\
\text { 30Je54 } \\
\text { 30Je54 }\end{array}$ & $\begin{array}{r}10,023 \\
1,657 \\
11,183 \\
26,048 \\
7,254\end{array}$ & $\begin{array}{r}3,444 \\
1,728 \\
1,348 \\
2,434 \\
873\end{array}$ & $\begin{array}{c}1,051,677 \\
448,687 \\
672,989 \\
417,753 \\
783,59417\end{array}$ & $\begin{array}{l}63,799 \\
10,636 \\
14,808 \\
15,367 \\
30,540\end{array}$ & $\begin{array}{r}275 \\
19 \\
16 \\
9 \\
52\end{array}$ & $\begin{array}{l}14,51318 \\
3,300^{18} \\
1,935 \\
1,906 \\
4,453^{18}\end{array}$ & $\begin{array}{l}643,329 \\
119,695 \\
143,423 \\
262,992 \\
132,038\end{array}$ & $\begin{array}{l}180,972 \\
15,301 \\
32,750 \\
34,501\end{array}$ & $\begin{array}{l}824,301 \\
121,19521 \\
158,724 \\
295,742 \\
166,539\end{array}$ \\
\hline $\begin{array}{l}\text { 11. Columbia }{ }^{6} \ldots \ldots \ldots \ldots \\
\text { 12. Cornell. } \ldots \ldots \ldots \ldots \ldots \\
\text { 13. Denver } \ldots \ldots \ldots \ldots \ldots \\
\text { 14. Florida } \ldots \ldots \ldots \ldots \\
\text { 15. Florida State } \ldots \ldots \ldots \ldots\end{array}$ & $\begin{array}{l}\text { 30Je54 } \\
\text { 30Je54 } \\
\text { 31Ag54 } \\
\text { 30Je54 } \\
\text { 30Je54 }\end{array}$ & $\begin{array}{l}9,997 \\
8,625 \\
4,469 \\
8,460 \\
5,316\end{array}$ & $\begin{array}{r}15,262 \\
1,365 \\
1,014 \\
1,166 \\
817\end{array}$ & $\begin{array}{l}2,069,795^{19} \\
1,674,735 \\
314,381 \\
564,464 \\
382,298\end{array}$ & $\begin{array}{l}62,740^{19} \\
64,775 \\
9,712 \\
36,979 \\
39,698\end{array}$ & $\begin{array}{l}123 \\
\ldots 28 \\
152 \\
98\end{array}$ & $\begin{array}{r}10,475 \\
11,780 \\
1,710 \\
3,369 \\
2,267\end{array}$ & $\begin{array}{r}863,986 \\
516,354 \\
99,405 \\
311,439 \\
202,959\end{array}$ & $\begin{array}{l}53,957 \\
10,616 \\
46,749 \\
11,420\end{array}$ & $\begin{array}{l}863,986 \\
570,311 \\
110,021 \\
358,188 \\
214,379\end{array}$ \\
\hline $\begin{array}{l}\text { 16. Fordham } \ldots \ldots \ldots \ldots \ldots \\
\text { 17. Georgia } \ldots \ldots \ldots \ldots \ldots \ldots \\
\text { 18. Harvard } \ldots \ldots \ldots \ldots \ldots \\
\text { 19. Hawaii } \ldots \ldots \ldots \ldots \ldots \\
\text { 20. Illinois } \ldots \ldots \ldots \ldots\end{array}$ & $\begin{array}{l}\text { 30Je54 } \\
\text { 30Je54 } \\
\text { 30Je54 } \\
\text { 30Je54 } \\
\text { 30Je54 }\end{array}$ & $\begin{array}{r}6,424 \\
4,175 \\
4,411 \\
4,403 \\
18,070\end{array}$ & $\begin{array}{r}2,246 \\
427 \\
5,647 \\
216 \\
3,058\end{array}$ & $\begin{array}{r}298,473 \\
311,193 \\
5,833,116 \\
247,310 \\
2,789,863\end{array}$ & $\begin{array}{r}10,123 \\
11,907 \\
130,169 \\
10,134 \\
109,377\end{array}$ & $\begin{array}{l}6 \\
34 \\
\ldots 12 \\
28 \\
625\end{array}$ & $\begin{array}{r}1,032 \\
2,551 \\
\cdots 7^{12} \\
3,878^{18} \\
13,774^{18}\end{array}$ & $\begin{array}{l}97,650 \\
146,355 \\
106,427^{12} \\
842,919\end{array}$ & $\begin{array}{l}18,000 \\
10,128 \\
\cdots,{ }^{12} \\
8,498 \\
29,742\end{array}$ & $\begin{array}{r}115,650 \\
156,483 \\
1,107,510 \\
114,925 \\
935,661\end{array}$ \\
\hline 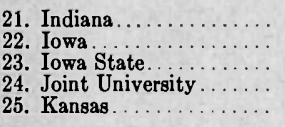 & $\begin{array}{r}\text { 30Je54 } \\
\text { 30Je54 } \\
\text { 30Je54 } \\
\text { 30Ap54 } \\
\text { 30Je54 }\end{array}$ & $\begin{array}{l}9,656 \\
5,881 \\
6,898 \\
3,367 \\
6,304\end{array}$ & $\begin{array}{r}1,972 \\
1,805 \\
882 \\
1,084 \\
853\end{array}$ & $\begin{array}{l}946,599 \\
826,838 \\
443,065 \\
655,274 \\
601,192^{16}\end{array}$ & $\begin{array}{l}36,523 \\
30,216 \\
11,873 \\
24,839 \\
60,416\end{array}$ & $\begin{array}{r}180 \\
113 \\
103 \\
32 \\
184\end{array}$ & $\begin{array}{l}11,581^{18} \\
5,110 \\
2,649 \\
3,994 \\
7,275^{18}\end{array}$ & $\begin{array}{l}326,895 \\
271,875 \\
151,688 \\
121,347 \\
204,312\end{array}$ & $\begin{array}{l}46,817 \\
45,525 \\
21,394 \\
30,966 \\
45,346\end{array}$ & $\begin{array}{l}373,712 \\
317,400 \\
173,082 \\
152,313 \\
249,658\end{array}$ \\
\hline $\begin{array}{l}\text { 26. Kentucky } \ldots \ldots \ldots \ldots \\
\text { 27. Louisiana State }{ }^{7} \ldots \ldots \ldots \\
\text { 28. Maine } \ldots \ldots \ldots \ldots \\
\text { 29. Maryland } \ldots \ldots \ldots \\
\text { 30. Miami (Florida) } \ldots \ldots \ldots\end{array}$ & $\begin{array}{r}\text { 30Je54 } \\
\text { 30Je54 } \\
\text { 30Je54 } \\
\text { 30Je54 } \\
\text { 31My54 }\end{array}$ & $\begin{array}{r}4,668 \\
6,223 \\
2,882 \\
10,912 \\
9,889\end{array}$ & $\begin{array}{r}596 \\
1,583 \\
77 \\
2,058 \\
429\end{array}$ & $\begin{array}{l}650,740^{16} \\
625,813 \\
260,703 \\
284,463 \\
380,877\end{array}$ & $\begin{array}{r}44,433 \\
34,683 \\
8,697 \\
14,721 \\
34,162\end{array}$ & $\begin{array}{r}160 \\
105 \\
16 \\
114 \\
42\end{array}$ & $\begin{array}{l}3,575 \\
5,7018 \\
1,451 \\
3,804 \\
2,517\end{array}$ & $\begin{array}{r}145,056 \\
252,334 \\
31,782 \\
152,074 \\
174,333\end{array}$ & $\begin{array}{r}15,076 \\
25,520 \\
8,475 \\
24,536 \\
19,842\end{array}$ & $\begin{array}{r}160,132 \\
277,854 \\
40,257 \\
176,610 \\
194,175\end{array}$ \\
\hline $\begin{array}{l}\text { 31. Michigan } \ldots \ldots \ldots \ldots \\
\text { 32. Michigan State } \ldots \ldots \ldots \\
\text { 33. Minnesota } \ldots \ldots \ldots \ldots \\
\text { 34. Mississippi } 10 \ldots \ldots \ldots \ldots \\
\text { 35. Mississippi State } \ldots \ldots \ldots\end{array}$ & $\begin{array}{l}\text { 30Je54 } \\
\text { 30Je54 } \\
\text { 30Je54 } \\
\text { 30Je54 } \\
\text { 30Je54 }\end{array}$ & $\begin{array}{r}10,456 \\
13,326 \\
16,133 \\
2,083 \\
2,557\end{array}$ & $\begin{array}{r}7,004 \\
1,453 \\
2,941 \\
176 \\
251\end{array}$ & $\begin{array}{r}2,304,434 \\
684,920 \\
1,763,728 \\
194,111 \\
171,710\end{array}$ & $\begin{array}{r}58,018 \\
70,619 \\
62,450 \\
9,530 \\
7,016\end{array}$ & $\begin{array}{r}104^{20} \\
69 \\
140 \\
59 \\
\mathbf{5 1}\end{array}$ & $\begin{array}{l}7,195 \\
4,690 \\
7,98918 \\
1,434 \\
1,326\end{array}$ & $\begin{array}{r}640,264 \\
135,260 \\
483,604 \\
\mathbf{6 7}, \mathbf{7 7 7} \\
\mathbf{5 7}, 052\end{array}$ & $\begin{array}{r}122,930 \\
62,732 \\
87,481 \\
8,302 \\
8,145\end{array}$ & $\begin{array}{l}763,194 \\
205,492^{22} \\
571,085 \\
76,079 \\
65,197\end{array}$ \\
\hline 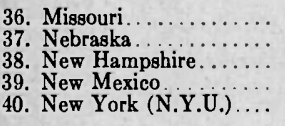 & $\begin{array}{l}\text { 30Je54 } \\
\text { 30Je54 } \\
\text { 30Je54 } \\
\text { 30Je54 } \\
\text { 30Je54 }\end{array}$ & $\begin{array}{r}6,578 \\
6,129 \\
2,738 \\
3,494 \\
16,480\end{array}$ & $\begin{array}{r}801 \\
642 \\
164 \\
669 \\
16,306\end{array}$ & $\begin{array}{r}708,679 \\
535,538 \\
211,845 \\
247,354 \\
1,017,226\end{array}$ & $\begin{array}{l}20,116 \\
19,038 \\
11,533 \\
16,471 \\
31,291\end{array}$ & $\begin{array}{c}200^{9} \\
140 \\
49 \\
52 \\
25\end{array}$ & $\begin{array}{l}3,000^{9} \\
5,883 \\
1,336 \\
5,790^{18} \\
5,000^{9}\end{array}$ & $\begin{array}{c}125,213 \\
212,529 \\
55,332 \\
79,353 \\
389,306^{8}\end{array}$ & $\begin{array}{l}18,036 \\
32,853 \\
11,903 \\
18,777 \\
\cdots \cdots\end{array}$ & $\begin{array}{r}143,249 \\
245,482 \\
67,235 \\
98,130 \\
389,306^{8}\end{array}$ \\
\hline $\begin{array}{l}\text { 41. North Carolina . . . . . } \\
\text { 42. North western } \ldots \ldots \ldots \ldots \\
\text { 43. Ohio State } \ldots \ldots \ldots \ldots \ldots \\
\text { 44. Oklahoma } \ldots \ldots \ldots \ldots \ldots \\
\text { 45. Oregon }{ }^{13} \ldots \ldots \ldots \ldots \ldots\end{array}$ & $\begin{array}{l}\text { 30Je54 } \\
\text { 31Ag54 } \\
\text { 30Je54 } \\
\text { 30Je54 } \\
\text { 30Je54 }\end{array}$ & $\begin{array}{r}3,871 \\
17,354 \\
16,944 \\
8,018 \\
3,512\end{array}$ & $\begin{array}{r}1,805 \\
4,407 \\
2,708 \\
1,085 \\
\mathbf{5 3 1}\end{array}$ & $\begin{array}{r}662,978 \\
1,146,163 \\
1,056,226 \\
504,382 \\
537,868\end{array}$ & $\begin{array}{l}29,473 \\
42,996 \\
45,479 \\
26,165 \\
26,172\end{array}$ & $\begin{array}{r}73 \\
87 \\
71 \\
359 \\
150\end{array}$ & $\begin{array}{c}4,500^{9} \\
12,850^{18} \\
9,006^{18} \\
4,989 \\
3,358\end{array}$ & $\begin{array}{l}256,939 \\
289,410 \\
400,292 \\
159,973 \\
183,663\end{array}$ & $\begin{array}{l}42,731 \\
60,155 \\
55,664 \\
28,885 \\
16,887\end{array}$ & $\begin{array}{l}299,670 \\
349,565 \\
455,956 \\
188,858 \\
200,550\end{array}$ \\
\hline 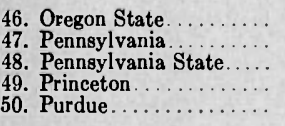 & $\begin{array}{l}\text { 30Je54 } \\
\text { 30Je54 } \\
\text { 30Je54 } \\
\text { 30Je54 } \\
\text { 30Je54 }\end{array}$ & $\begin{array}{r}4,435 \\
9,920 \\
10,192 \\
2,892 \\
8,243\end{array}$ & $\begin{array}{r}429 \\
5,292 \\
1,337 \\
537 \\
1,496\end{array}$ & $\begin{array}{r}296,305 \\
1,371,193 \\
386,296 \\
1,275,703 \\
353,929\end{array}$ & $\begin{array}{l}14,368 \\
47,863 \\
18,368 \\
34,018 \\
18,607\end{array}$ & $\begin{array}{r}115 \\
106 \\
76 \\
67 \\
21\end{array}$ & $\begin{array}{c}2,825 \\
5,773 \\
4,278 \\
20,000^{18} \\
5,133\end{array}$ & $\begin{array}{l}109,662 \\
428,096 \\
186,131 \\
308,085 \\
189,629\end{array}$ & $\begin{array}{l}59,702 \\
33,043 \\
54,491 \\
25,853 \\
23,397\end{array}$ & $\begin{array}{l}169,364 \\
461,139 \\
240,622 \\
333,938 \\
213,026\end{array}$ \\
\hline 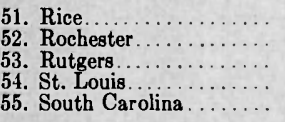 & $\begin{array}{r}\text { 30Je54 } \\
\text { 30Je54 } \\
\text { 30Je54 } \\
\text { 31Ag54 } \\
\text { 30Je54 }\end{array}$ & $\begin{array}{l}1,429 \\
4,528 \\
8,744 \\
5,753 \\
3,073\end{array}$ & $\begin{array}{r}200 \\
679 \\
1,737 \\
2,508 \\
398\end{array}$ & $\begin{array}{l}249,736 \\
580,609 \\
698,585 \\
498,075 \\
299,105\end{array}$ & $\begin{array}{l}13,740 \\
17,122 \\
33,294 \\
28,137 \\
14,058\end{array}$ & $\begin{array}{l}10 \\
45 \\
33 \\
53 \\
50\end{array}$ & $\begin{array}{l}2,529 \\
3,163 \\
3,200 \\
3,592 \\
1,200\end{array}$ & $\begin{array}{r}65,808 \\
158,083 \\
299,046 \\
115,160 \\
84,659\end{array}$ & $\begin{array}{l}20,772 \\
14,642 \\
28,862 \\
15,046 \\
16,966\end{array}$ & $\begin{array}{l}86,580 \\
172,725 \\
327,908 \\
142,206^{23} \\
101,625\end{array}$ \\
\hline 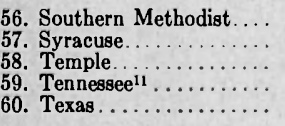 & $\begin{array}{l}\text { 30Je54 } \\
\text { 30Je54 } \\
\text { 30Je54 } \\
\text { 30Je54 } \\
\text { 31Ag54 }\end{array}$ & $\begin{array}{r}3,532 \\
6,999 \\
11,574 \\
4,892 \\
11,868\end{array}$ & $\begin{array}{r}885 \\
2,087 \\
5,969 \\
946 \\
1,958\end{array}$ & $\begin{array}{r}363,571 \\
387,694 \\
398,923 \\
307,117 \\
1,095,284\end{array}$ & $\begin{array}{l}18,809 \\
12,514 \\
25,450 \\
12,487 \\
45,531\end{array}$ & $\begin{array}{r}31 \\
35 \\
22 \\
26 \\
160\end{array}$ & $\begin{array}{l}1,678 \\
2,525 \\
2,841 \\
2,336 \\
7,200^{18}\end{array}$ & $\begin{array}{l}101,840 \\
160,844 \\
177,327 \\
130,054 \\
260,344\end{array}$ & $\begin{array}{r}14,475 \\
25,377 \\
19,718 \\
8,588 \\
82,155\end{array}$ & $\begin{array}{l}116,315 \\
186,221 \\
197,045 \\
138,642 \\
342,499\end{array}$ \\
\hline 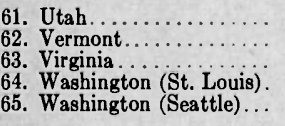 & $\begin{array}{l}\text { 30Je54 } \\
\text { 30Je54 } \\
\text { 30Je54 } \\
\text { 30Je54 } \\
\text { 30Je54 }\end{array}$ & $\begin{array}{c}5,977 \\
2,518 \\
3,834^{15} \\
10,023 \\
11,251\end{array}$ & $\begin{array}{r}1,165 \\
245 \\
1,002 \\
1,797\end{array}$ & $\begin{array}{l}307,909 \\
200,000^{9} \\
746,834 \\
\mathbf{5 7 7}, 690 \\
842,928\end{array}$ & $\begin{array}{r}17,160 \\
4,977 \\
37,018 \\
14,812 \\
49,255\end{array}$ & $\begin{array}{r}53 \\
10 \\
127 \\
13 \\
215\end{array}$ & $\begin{array}{c}5,951^{18} \\
1,400 \\
3,751 \\
\cdots \ldots 1^{12} \\
9,691^{18}\end{array}$ & $\begin{array}{r}92,292 \\
65,849 \\
236,919 \\
141,291 \\
368,224\end{array}$ & $\begin{array}{r}32,590 \\
7,029 \\
24,807 \\
27,965 \\
82,801\end{array}$ & $\begin{array}{r}124,882 \\
72,878 \\
261,726 \\
169,256 \\
451,025\end{array}$ \\
\hline 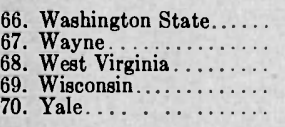 & $\begin{array}{r}\text { 31 Mr54 } \\
\text { 30Je54 } \\
\text { 30Je54 } \\
\text { 30Je54 } \\
\text { 31My54 }\end{array}$ & $\begin{array}{r}4,590 \\
13,527 \\
4,377 \\
10,821 \\
3,993\end{array}$ & $\begin{array}{r}466 \\
2,881 \\
573 \\
2,525 \\
3,135\end{array}$ & $\begin{array}{r}650,000 \\
479,157 \\
283,345 \\
947,896 \\
4,245,583\end{array}$ & $\begin{array}{l}12,251 \\
35,193 \\
13,808 \\
38,310 \\
59,611\end{array}$ & $\begin{array}{l}209 \\
94 \\
96 \\
17 \\
50^{24}\end{array}$ & $\begin{array}{c}5,000^{18} \\
3,611 \\
1,906 \\
11,000^{18} \\
5,829\end{array}$ & $\begin{array}{l}175,572 \\
289,000 \\
140,050 \\
433,414 \\
636,541\end{array}$ & $\begin{array}{r}31,717 \\
48,000 \\
25,659 \\
66,435 \\
\cdots \cdots\end{array}$ & $\begin{array}{l}207,289 \\
337,009 \\
165,709 \\
499,849 \\
636,541\end{array}$ \\
\hline $\begin{array}{l}\text { High. . . . . . } \\
\text { Median . . . } \\
\text { Low . . . . . } \\
\text { N14 . . . . . }\end{array}$ & $\begin{array}{l}\ldots \ldots \ldots \\
\cdots \ldots \ldots \\
\cdots \ldots \ldots \\
\ldots \ldots\end{array}$ & $\begin{array}{c}26,048 \\
6,263 \frac{1}{2} \\
1,429 \\
70\end{array}$ & $\begin{array}{r}16,306 \\
1,737 \\
77 \\
69\end{array}$ & $\begin{array}{r}5,833,116 \\
551,166 \\
171,710 \\
70\end{array}$ & $\begin{array}{r}130,169 \\
25,811 \\
4,977 \\
70\end{array}$ & $\begin{array}{r}625 \\
63 \\
6 \\
68\end{array}$ & $\begin{array}{r}23,177 \\
3,168 \\
824 \\
68\end{array}$ & $\begin{array}{r}1,192,004 \\
161,974 \\
31,782 \\
69\end{array}$ & $\begin{array}{r}241,027 \\
25,659 \\
7,029 \\
65\end{array}$ & $\begin{array}{r}1,433,031 \\
195,610 \\
40,257 \\
70\end{array}$ \\
\hline
\end{tabular}

1 Central library and all agencies. 2 Resident and special students (no person counted twice). 3 Includes supplies, transportation, and all other noncapital expenditures not specifically reported. 4 Exclusive of capital expenditures and those for auxiliary enterprises such as dormitories, cafeterias, etc. 7 Includes Schools of Law and Medicine for first time. 8 Excludes NYU-Bellevue Medical Center. 9 Estimate. 10 Excludes School of Law and Medicine. 
Statistics: 1953-54 (Group I)

Expenditures

\begin{tabular}{c}
\hline Book \\
and \\
Rejated \\
Materi \\
\\
90,2 \\
47,196 \\
33,7 \\
78,4 \\
325,62
\end{tabular}

315,8

42,06

55,17

73,300

368,10$$
33 \text {, }
$$$$
119,62
$$$$
58,2
$$$$
\begin{array}{r}
74,4 \\
416,3
\end{array}
$$$$
416,350
$$$$
\text { ....... }
$$

216,00

137,7
69,4

183,8

85,1
165,0
23,0
88,9
105,1

242,8

251,3

20,106

92,7

92,7
25,

25,921
74,108

$\begin{array}{lll}148,172 & 18,094 & 166,26 \\ 185,062 & 15,000^{25} & 197,290 \\ 1212 & 200,06\end{array}$

$\begin{array}{rlr}121,043 & 18,214 & 139,25 \\ 77,862 & 14,113 & 91,975\end{array}$

70,4

200,0

76,363
130,605

116,351

$15,216 \quad 85,632$

$\begin{array}{rr}47,833 & 247,897 \\ 11,495 & 87,858\end{array}$

$\begin{array}{rr}11,495 & 87,858 \\ 21,081 & 151,68\end{array}$

$13,122 \quad 129,473$

$46,259 \quad 10,000 \quad 56,259$

$86,551_{12} \quad 14,341_{12} \quad 100,89$

$70,977^{12} \quad 13,538{ }^{12} \quad 140,700$

$\begin{array}{rrr}62,067 & 7,913 & 69,98 \\ 51,227 & 10,035 & 61,26 \\ 64,409 & 9,678 & 74,08\end{array}$

$\begin{array}{rrr}64,409 & 9,678 & 74,08 \\ 104,696 & 11,415 & 116,111\end{array}$

$170,806 \quad 29,251 \quad 200,057$

81,581

21,3

$\begin{array}{rrr}21,343 & 5,569 & 26,9 \\ 94,406 & 19,763 & 114,169\end{array}$

ii $9,287^{12} \quad 46,307 \quad r 7^{12} \quad 165,745$

\begin{tabular}{ccr}
$\mathbf{7 4}, 986$ & \multicolumn{1}{c}{$\mathbf{6 , 6 4 6}$} & $\mathbf{8 1}, \mathbf{5 3 2}$ \\
$\mathbf{1 5 6 , 8 9 5}$ & 10,761 & 167,656 \\
57,421 & 13,241 & 70,662 \\
172,423 & 38,523 & 210,946 \\
$\ldots \ldots \ldots$ & $\ldots \ldots .12$ & 423,837
\end{tabular}

$\begin{array}{rccrr}416,350 & 91,424 & 506,422 & 257,295 & 2,000,945 \\ 81,581 & 13,928 \frac{1}{2} & 102,499 & 16,998 & 318,177 \\ 16,441 & 1,922 & 24,708 & 896 & 66,126 \\ 63 & 62 & 70 & 69 & 70\end{array}$
Per Student tional tures $\begin{gathered}\text { Expendi- } \\ \text { tures }\end{gathered}$

Expendi- $_{\text {tures }}{ }^{3}$
Expendi-
tures

$\$ 22,652$

12,505
8,077

16,663

150,867

$\$ 321,652$

160,276

277,156

$2,000,945$

81,151
9,786

5,625

$1,295,186$

173,043
230,491

383,821
260,422

7,03

$1,357,576$

63,675

44,100
3,597

23,864

14,070

855,116

530,921

6,670

12,858

257,295
5,983

186,920

253,477

871,227
175,292

$43,852 \quad 1,415,513$

2,200

27,549
10,788

10,788
10,784

651,712

499,176

278,969

246,317
493,518

8,685

271,023

483,840

$\begin{array}{rr}896 & 66,126 \\ 12,617 & 292,020 \\ 11,537 & 326,474\end{array}$

$1,098,104$

21,354

57,662

4,654
7,741

19,415

21,586

3,998
5,592

5,592
$29,120^{8}$

24,211
31,589

31,589
31,542

20,995

8, 611

31,347

16,998

29,342

7,000

13,667
18,125

18,125

3,319

5,411

10,235

$\begin{array}{r}\mathbf{8}, 108 \\ \hline\end{array}$

23,825

17,188

11,991

29,225
17,099

37,303

395,721
922,129

108,132
97,646

268,020

390,081

102,37

156,504
$510,221^{8}$

490,147

$\mathbf{5 7 8}, 449$
687,560

349,110

314,702

263,607

740,383

345,478

519,219
371,841

149,839

287,284
486,733

244,081
175,546

191,706

252,790

262,861

566,381

237,647

111,781
405,120

285,100

653,922

$9,403 \quad 298,224$

$18,736 \quad 523,392$

$14,246 \quad 250,617$

$31,806 \quad 742,610$

$\begin{array}{rr}56.85 & \$ 6,053,346 \\ 30.16 & 4,244,022 \\ 14.84 & 6,046,994 \\ 89.68 & 4,304,008 \\ 130.55 & \end{array}$

96.17

51.12
18.39

13.48

32.04

53.74

85.59

28.00

61.32

21.44

55.00

186.04
37.95

67.00

56.00

64.95

35.85
43.19

68.96

51.40

61.98

22.00

22.51
31.64

62.89

25.98

48.34
47.86

47.86
34.77

36.30

37.76
37.54

37.54

37.59
$13.11^{3}$

86.35

$48.00^{26}$

34.98

38.35
77.83

54.19

48.67

29.96

122.26

38.18

91.98

86.50

46.43
29.54

29.54
50.57

33.67

27.82
15.87

15.87
45.00

40.96

33.27

40.45

25.85

50.12

58.98

92.87

50.62

55.64
140.35

186.04

$47.14 \frac{1}{2}$
13.11

13.11
70

$14,672,683$

$3,220,207$

$6,957,293$

$8,845,168$
$6,235,203$

$28,671,987$

$3,130,050$

$14,522,851$
$5,320,987$

$5,000,000$

$3,849,507$

$34,000,248$

$4,392,100$

12

$10,650,956$

$11,796,364$

$9,000,000^{9}$

$13,018,421$

$3,762,097$

$17,600,510$
$5,797,680$

$36,309,760$

$34,832,607$

$2,178,838$
$5,872,839$

$10,769,267$

$11,668,796$

$2,975,126$

$14,197,633$

$28,975,155$

$5,708,325^{23}$
$3,943,274$

$\ldots \ldots \ldots{ }^{12}$

$22,386,125$

$9,583,747$
$18,831,908$

$1,600,000$

$14,768,234$

$4,639,326$
$2,655,963$

$3,323,454$

$7,251,972$

$6,337,614$

$6,978,704$

$3,853,425$

$5,800,000$

$9,278,074$
$13,928,255$

ii $, 326,228$

Ratio of

Library Ex-

penditures to

Total Insti-

penditures

(Per Cent)

5.3
3.8

3.8
3.9

6.4

8.8

5.3
3.3

3.3
4.3

4.1

2.9
4.8
3.6
6.7

3.7

3.7
6.6

5.5

3.1

4.6

2.3
4.4

3.0

3.7

1.7

3.0

2.6
4.9
1.6

2.5

3.3

2.7
5.3

$2.2^{8}$

4.0
2.3
5.5

7.9

1.5

5.4

8.1

3.3
5.2
6.6

5.8

3.9

4.1 
Salaries as of September 1, 1954

Library ${ }^{1}$

1. Alabama

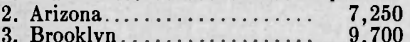

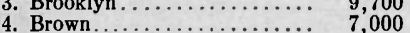

5. California (Berkeley)........ 14,700

6. California (Los Angeles) ..... 12,600

7. Catholic U. of America...... 6,000

8. Cincinnati

9. City College (N.Y.)......... 9,950

10. Colorado ${ }^{3} \ldots \ldots \ldots \ldots \ldots \ldots \ldots, 8,800$

11. Columbia ${ }^{4} \ldots \ldots \ldots \ldots \ldots \ldots \ldots \ldots \ldots$

12. Cornell $\ldots \ldots \ldots \ldots \ldots \ldots \ldots, \ldots \ldots{ }_{12}^{12}$

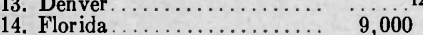

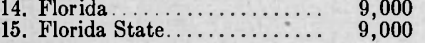

16. Fordham ............. 6,500

6,500
8,100

18. Harvard

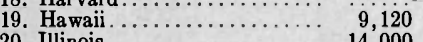

20. Illinois . . . . . . . $14,00 \ldots \ldots \ldots, \quad$

21. Indiana

22. Iowa

$10,700^{12}$

23. Iowa State............... $\quad 9,800$

25. Joint University .......... $97^{12}$

9,600

26. Kentucky.

26. Kentucky $\ldots \ldots \ldots \ldots \ldots \ldots, \quad 7,000^{15}$

28. Maine.

6,400
6,950

30. Miami (Flo:ida).......... 9,000

31. Michigan

32. Michigan State

33. Minnesota

10,750

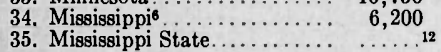

36. Missouri

7,800

37. Nebraska

38. New Hampshirew Mexico

40. New York (N.Y.U.

41. North Carolina

43. Ohio State

44. Oklahom

45. Oregon?

.................

6. Oregon State

6. Oregon State $\ldots \ldots \ldots \ldots \ldots \ldots$
7. Pennsvlvania $\ldots \ldots \ldots \ldots \ldots$

49. Princeton

50. Purdue................. $12,00{ }^{12}$

51. Rice.

$\ldots \ldots \ldots \ldots \ldots \ldots, 8,000$

52. Rocheste

53. Rutgers.

54. St. Louis . . . . . . . . . . . . 12,000

55. South Carolina ........... $6,6,500$

56. Southern Methodist....... 6, 636

57. Syracuse

58. Temple.

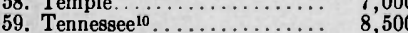

60. Texas .................... $9,6,620$

61. Utah

...

7,800

63. Virginia

(St Lou:s)

65. Washington (Seattle)....... 11,004

66. Washington State........ 10,000

67. Wayne
68. West Virginia................

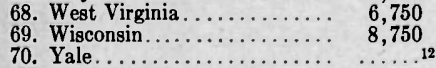

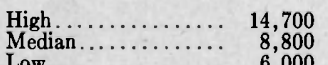

$\begin{array}{lr}\text { Median ............. } & 8,800 \\ \text { Low............. } & \mathbf{6}, 000 \\ \text { N14 }\end{array}$

53
Associate or

Assistant.

Minimum Maximum

$\$ . \ldots$

$\cdots \cdots$

\$.....

Minimum Maximum Number

$\ddot{7}, 008$

$\ddot{9}, \ddot{3} \dot{8}$

6,360
4,200

6,530

6,300

$5,371^{12}$

6,700

5,460

4,700

5,800

$\ddot{5}, 400$

6,800

7,500

7,200

5,400

6,070

….

10,000

0,000

5,900
7,400

.......

4,700

4,700
5,900
5,200

$\ldots \ldots$

5,094

6,094

, 300

7,224

......

9,500

$\begin{array}{ll}\dddot{6}, 200 & 7,000 \\ 6,408 & 6,408\end{array}$

$\ldots \ldots$

4,800

6,534

5,400

...

4,380

6,000

6,400

6,400
4,600

.......

……

8,900

$\therefore, 020$

8,410

$\begin{array}{rr}10,000 & 11,000 \\ 6,035 & 7,618\end{array}$

$4,200 \quad 4,700$
$\$ 4,000$

8,112

7,618

.....

7,200

.....

6,400

9,400

$\ldots{ }^{12}$

.....

6,700

8,000

.....

11,000

8,700

.......

6,000

$\ldots \ldots$

6,093

7,632

6,300

7,000

$\because, 854$

.....

.

.......

$\cdots$

....

4,700

.....

.....

700
21

$\$ 5,500$

4,600

5,938

3,600
4,296

6,250

5,000
8,316

6,060

3,300

4,068
5,554

8,112
4,320

4,320
5,004

6,250
4,020

$4_{428}^{12}$

4,428
4,250

4,250
4,500

4,500
4,300

$\ldots{ }^{12}$

6,432
4,500

4,500
6,050

6,050
5,700

4,000

3,825

6,100

4,700

$\ddot{4}, 575$

5,600

4,500

5,000
2,520

2,520
4,200

3,816

3,500

4,400

5,800

5,800

3,900

5,448
3,300

3,300
3,750

4,200

5,000
4,200

4,500
$5,400^{8}$

5,750

5,000

4,200

4,600

4,380
5,975

4,813

5,000

7,000

5,220

6,600
4,300

4,300

4,500

5,000

5,500
$5,400^{8}$

3,366

3,800

5,424
3,780

4,300

9,935

5,400

6,624
5,300

5,300
5,700

5,000

3,650
4,680

5,600

5,400

5,196

6,000
5,500

4,500

4,000

4,900
5,478

5,478
4,800

4,400

4,900

6,798
5,400

5,400
3,800

3,420
3,960

3,960
4,020

4,400
3,648

4,032

4,080

4,620

4,800
5,120

3,200

5,400

4,380
5,820

4,700

6,144

4,740
6,120

$\begin{array}{ll}\ldots \ldots & 5,700 \\ \cdots \ldots & 6,012\end{array}$

6,500

4,750

4,650
6,774

$6,100 \quad 9,935$

4,296
2,520

2,520

5,220

3,800
63
$4,175 \quad 4,300$

8
3
5
5
28

5
4
4
8
9

Head Librarian

and Departmental (Prof.)

Minimum Maximum Number

$\$, 100$

.....

$3,120 \quad 3,600 \quad{ }_{18}$

$3,540 \quad 6,672$

3,000
2,400

$4452^{18} \quad \cdots .^{18}$

$\ldots{ }^{12} \quad \ldots \ldots 12 \quad{ }^{12}$

3,516

4,35

3,600
3,700

6,000

4,500

6,900
5,200 
Statistics: 1953-54 (Group I)

Salaries as of September 1,1954

\begin{tabular}{|c|c|c|c|c|c|c|c|c|c|c|c|c|}
\hline \multicolumn{3}{|c|}{$\begin{array}{l}\text { All Other Professional } \\
\text { Assistants }\end{array}$} & \multicolumn{3}{|c|}{$\begin{array}{l}\text { All Nonprofessional } \\
\text { Assistants }\end{array}$} & \multicolumn{3}{|c|}{$\begin{array}{l}\text { Total Number of Employees in } \\
\text { Full-Time Equivalent }{ }^{2} \\
\text { Pro- Nonpro- }\end{array}$} & \multicolumn{2}{|c|}{$\begin{array}{l}\text { Length of } \\
\text { Work Week }\end{array}$} & $\begin{array}{l}\text { Hours of } \\
\text { Student } \\
\text { Assistanc }\end{array}$ & \\
\hline $\begin{array}{r}\$ 2,700 \\
3,125 \\
3,700 \\
2,760 \\
3,372\end{array}$ & $\begin{array}{r}\$ 3,900 \\
3,700 \\
5,200 \\
3,600 \\
6,672\end{array}$ & $\begin{array}{l}28 \frac{1}{2}^{\circ} \\
7^{\circ} \\
17 \\
12 \\
97\end{array}$ & $\begin{array}{r}\$ 1,500 \\
2,650 \\
2,815 \\
1,740 \\
2,400\end{array}$ & $\begin{array}{r}\$ 2,400 \\
3,300 \\
4,905 \\
3,000 \\
6,204\end{array}$ & $\begin{array}{r}14 \\
10 \\
19 \\
39 \\
183\end{array}$ & $\begin{array}{c}38 \frac{1}{2} \\
12 \frac{1}{2} \\
25 \frac{1}{2} \\
21 \\
126 \frac{1}{4}\end{array}$ & $\begin{array}{r}14 \\
10 \\
19 \\
37 \\
173\end{array}$ & $\begin{array}{r}52 \frac{1}{2} \\
22 \frac{1}{3} \\
44 \frac{1}{3} \\
58 \\
299 \frac{1}{4}\end{array}$ & $\begin{array}{l}39 \\
39 \\
36 \frac{1}{4} \\
38 \\
40\end{array}$ & $\begin{array}{l}.12 \\
5 \frac{1}{2} \\
5 \\
5 \frac{1}{2} \\
5\end{array}$ & $\begin{array}{r}50,000 \\
24,160 \\
25,395 \\
19,561 \\
191,567\end{array}$ & 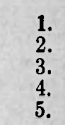 \\
\hline $\begin{array}{l}3,540 \\
3,000 \\
3,096 \\
4,000 \\
3,420\end{array}$ & $\begin{array}{l}6,672 \\
3,720 \\
4,092 \\
5,200 \\
4,092\end{array}$ & $\begin{array}{r}65 \\
11 \\
10 \\
32 \\
8\end{array}$ & $\begin{array}{l}2,400 \\
1,980 \\
1,992 \\
2,400 \\
2,064\end{array}$ & $\begin{array}{l}6,060 \\
2,700 \\
2,652 \\
3,145 \\
3,564\end{array}$ & $\begin{array}{r}85 \\
20 \\
22 \\
9 \\
18\end{array}$ & $\begin{array}{l}80 \\
18 \\
17 \\
48 \\
20\end{array}$ & $\begin{array}{l}85 \\
20 \\
30 \frac{1}{2} \\
13 \\
18\end{array}$ & $\begin{array}{l}165 \\
38 \\
47 \frac{1}{2} \\
61 \\
38\end{array}$ & $\begin{array}{l}40 \\
39 \\
40 \\
36 \frac{1}{2} \\
40\end{array}$ & $\begin{array}{l}5 \\
5 \\
6 \\
5 \\
5\end{array}$ & $\begin{array}{r}153,366 \\
7,500 \\
21,269 \\
31,076 \\
43,126\end{array}$ & \\
\hline $\begin{array}{l}3,100 \\
3,312 \\
3,320 \\
3,400 \\
3,500\end{array}$ & $\begin{array}{l}4,200 \\
5,508 \\
3,800 \\
4,950 \\
4,500\end{array}$ & $\begin{array}{l}47 \frac{1}{2} \\
53 \\
8 \\
30 \\
20\end{array}$ & $\begin{array}{l}1,800 \\
2,184 \\
1,920 \\
1,800 \\
2,200\end{array}$ & $\begin{array}{l}3,600 \\
3,948 \\
2,970 \\
3,600 \\
3,200\end{array}$ & $\begin{array}{c}220 \\
78 \frac{1}{2} \\
17 \\
53 \frac{1}{4} \\
20\end{array}$ & $\begin{array}{l}89 \\
84 \frac{1}{2} \\
18 \\
44 \\
34\end{array}$ & $\begin{array}{c}22711 \\
84 \frac{1}{2} \\
17 \\
53 \frac{1}{6} \\
20\end{array}$ & $\begin{array}{c}316 \\
169 \\
35 \\
97 \frac{1}{2} \\
54\end{array}$ & $\begin{array}{l}38 \frac{1}{2} \\
39 \\
37 \frac{1}{2} \\
38 \frac{1}{2} \\
40\end{array}$ & $\begin{array}{l}5 \\
5 \\
5 \\
5 \frac{1}{2} \\
6\end{array}$ & $\begin{array}{l}68,900 \\
15,829 \\
67,391 \\
19,311\end{array}$ & $\begin{array}{l}14 \\
15\end{array}$ \\
\hline $\begin{array}{l}3,380 \\
3,250 \\
2,700 \\
2,875 \\
3,600\end{array}$ & $\begin{array}{l}4,175 \\
3,800 \\
9,500 \\
4,200 \\
6,000\end{array}$ & $\begin{array}{c}12 \\
15 \frac{1}{2} \\
111 \frac{1}{3} \\
11 \\
95\end{array}$ & $\begin{array}{l}2,080 \\
1,850 \\
1,320 \\
2,040 \\
1,920\end{array}$ & $\begin{array}{l}3,120 \\
2,500 \\
5,000 \\
3,355 \\
4,020\end{array}$ & $\begin{array}{c}20 \\
25 \frac{1}{2} \\
220 \frac{2}{3} \\
16 \\
111 \frac{3}{4}\end{array}$ & $\begin{array}{c}15 \\
26 \frac{1}{2} \\
137 \frac{1}{3} \\
17 \\
132\end{array}$ & $\begin{array}{c}20 \\
25 \frac{1}{2} \\
220 \frac{2}{3} \\
16 \\
110 \frac{3}{4}\end{array}$ & $\begin{array}{c}35 \\
52 \\
358 \\
33 \\
242 \frac{3}{4}\end{array}$ & $\begin{array}{l}35 \\
39 \\
35 \\
40 \\
39\end{array}$ & $\begin{array}{l}5 \\
6 \\
5 \\
6 \\
5\end{array}$ & $\begin{array}{r}18,259 \\
15,029 \\
60,000 \\
14,655 \\
105,929\end{array}$ & $\begin{array}{l}1 \\
17\end{array}$ \\
\hline $\begin{array}{l}3,200 \\
3,600 \\
3,540 \\
1,800 \\
3,300\end{array}$ & $\begin{array}{l}5,000 \\
4,600 \\
4,200 \\
3,300 \\
6,000\end{array}$ & $\begin{array}{c}23 \\
17 \frac{1}{2} \\
12 \frac{1}{2} \\
9 \\
28\end{array}$ & $\begin{array}{l}2,160 \\
2,100 \\
1,860 \\
1,590 \\
1,732\end{array}$ & $\begin{array}{l}3,573 \\
3,300 \\
3,060 \\
2,700 \\
4,800\end{array}$ & $\begin{array}{l}53 \\
484 \\
27 \\
15 \\
32\end{array}$ & $\begin{array}{l}40 \\
30 \frac{3}{4} \\
19 \frac{1}{2} \\
26 \\
37 \frac{1}{4}\end{array}$ & $\begin{array}{l}53 \frac{1}{3} \\
47 \frac{3}{4} \\
27 \\
14 \frac{1}{2} \\
27 \frac{1}{4}\end{array}$ & $\begin{array}{l}93 \frac{1}{2} \\
78 \frac{1}{2} \\
46 \frac{1}{2} \\
40 \frac{1}{2} \\
64 \frac{1}{2}\end{array}$ & $\begin{array}{l}38 \frac{1}{2} \\
40 \\
40 \\
40 \\
40\end{array}$ & $\begin{array}{l}5 \frac{1}{2} \\
5 \\
5 \\
5 \\
5\end{array}$ & $\begin{array}{l}49,7^{12} \\
23,345 \\
61,932 \\
66,307\end{array}$ & $\begin{array}{l}21 . \\
22 . \\
23 . \\
24 . \\
25 .\end{array}$ \\
\hline $\begin{array}{l}2,400 \\
2,600 \\
2,460 \\
3,572 \\
3,300\end{array}$ & $\begin{array}{l}3,672 \\
5,863 \\
4,300 \\
4,813 \\
4,048\end{array}$ & $\begin{array}{r}14 \\
19 \\
5 \\
19 \\
11\end{array}$ & $\begin{array}{l}1,644 \\
2,040 \\
1,664 \\
2,717 \\
1,920\end{array}$ & $\begin{array}{l}2,196 \\
3,600 \\
2,405 \\
3,225 \\
2,700\end{array}$ & $\begin{array}{c}23 \\
30 \\
4 \\
17 \frac{1}{2} \\
39\end{array}$ & $\begin{array}{r}28 \\
47 \\
6 \\
25 \\
20\end{array}$ & $\begin{array}{c}26 \\
30 \\
6 \\
16 \frac{1}{2} \\
38 \frac{1}{2}\end{array}$ & $\begin{array}{l}54 \\
77 \\
12 \\
41 \frac{1}{2} \\
58 \frac{1}{2}\end{array}$ & $\begin{array}{l}39 \\
39 \\
39 \\
37 \\
38 \frac{3}{4}\end{array}$ & $\begin{array}{l}6 \\
5 \frac{1}{2} \\
5 \\
5 \\
5\end{array}$ & $\begin{array}{l}30,000^{9} \\
41,532 \\
10,051 \\
30,670 \\
25,836\end{array}$ & $\begin{array}{l}29 \\
30\end{array}$ \\
\hline $\begin{array}{l}3,600 \\
3,240 \\
3,480 \\
2,900 \\
2,700\end{array}$ & $\begin{array}{l}6,200 \\
4,440 \\
5,160 \\
3,200 \\
3,600\end{array}$ & $\begin{array}{r}78 \\
14 \\
68 \\
5 \\
6\end{array}$ & $\begin{array}{l}2,520 \\
2,640 \\
2,100 \\
1,800 \\
1,750\end{array}$ & $\begin{array}{l}4,860 \\
4,380 \\
3,720 \\
2,560 \\
2,400\end{array}$ & $\begin{array}{r}53 \\
21 \\
44 \\
5 \\
19\end{array}$ & $\begin{array}{c}102 \frac{1}{2} \\
25 \\
91 \\
12 \\
12\end{array}$ & $\begin{array}{c}51 \\
21 \\
44 \\
6 \frac{1}{2} \\
7\end{array}$ & $\begin{array}{c}153 \frac{1}{2} \\
46 \\
135 \\
18 \frac{1}{2} \\
19\end{array}$ & $\begin{array}{l}40 \\
40 \\
40 \\
38 \frac{1}{2} \\
41 \frac{1}{2}\end{array}$ & $\begin{array}{l}5 \\
5 \\
5 \\
6 \\
5 \frac{1}{2}\end{array}$ & $\begin{array}{l}91,779 \\
62,732 \\
89,143 \\
16,603 \\
14,809\end{array}$ & $\begin{array}{l}31 . \\
32 . \\
33 . \\
34 . \\
35 .\end{array}$ \\
\hline $\begin{array}{l}3,000 \\
3,600 \\
3,200 \\
3,900 \\
3,300^{8}\end{array}$ & $\begin{array}{l}3,600 \\
5,000 \\
3,500 \\
4,500 \\
6,300^{8}\end{array}$ & $\begin{array}{c}10 \\
24 \\
2 \\
4 \\
27^{8}\end{array}$ & $\begin{array}{l}1,560 \\
1,800 \\
1,700 \\
2,200 \\
1,800^{8}\end{array}$ & $\begin{array}{l}2,500 \\
4,050 \\
2,300 \\
2,480 \\
3,600^{8}\end{array}$ & $\begin{array}{c}19 \\
31 \\
9 \\
8 \frac{1}{2} \\
91^{8}\end{array}$ & $\begin{array}{c}21 \\
34 \frac{1}{2} \\
9 \\
12 \\
36^{8}\end{array}$ & $\begin{array}{c}20 \\
30 \frac{1}{2} \\
7 \\
8 \frac{1}{2} \\
91^{8}\end{array}$ & $\begin{array}{c}41 \\
65 \\
16 \\
20 \frac{1}{2} \\
127^{8}\end{array}$ & $\begin{array}{l}39 \\
41 \\
40 \\
39 \\
38^{8}\end{array}$ & $\begin{array}{l}5 \frac{1}{2} \\
.12 \\
5 \\
5 \\
.12\end{array}$ & $\begin{array}{l}26,735 \\
43,909 \\
18,970 \\
22,320 \\
\ldots \ldots \ldots\end{array}$ & $\begin{array}{l}36 . \\
37 . \\
38 . \\
39 . \\
40 .\end{array}$ \\
\hline $\begin{array}{l}2,700 \\
3,000 \\
3,192 \\
3,000 \\
3,250\end{array}$ & $\begin{array}{l}4,842 \\
5,500 \\
5,124 \\
4,000 \\
4,200\end{array}$ & $\begin{array}{l}36 \\
27 \\
43 \\
14 \\
15 \frac{1}{2}\end{array}$ & $\begin{array}{l}1,709 \\
1,896 \\
2,208 \\
1,800 \\
1,956\end{array}$ & $\begin{array}{l}3,159 \\
3,000 \\
6,300 \\
2,520 \\
3,264\end{array}$ & $\begin{array}{l}25 \\
53 \\
90 \\
23 \\
26 \frac{1}{4}\end{array}$ & $\begin{array}{l}57 \\
45 \\
54 \\
29 \\
28 \frac{1}{2}\end{array}$ & $\begin{array}{l}25 \\
53 \\
96 \\
23 \\
26 \frac{1}{4}\end{array}$ & $\begin{array}{c}82 \\
98 \\
150 \\
52 \\
54_{4}^{\frac{3}{4}}\end{array}$ & $\begin{array}{l}39 \\
37 \frac{1}{2} \\
40 \\
40 \\
40\end{array}$ & $\begin{array}{l}5 \frac{1}{2} \\
5 \\
5 \frac{1}{2} \\
5 \\
5\end{array}$ & $\begin{array}{l}55,642^{16} \\
57,790 \\
68,000^{9} \\
48,000^{9} \\
19,269\end{array}$ & $\begin{array}{l}41 . \\
42 . \\
43 . \\
44 . \\
45 .\end{array}$ \\
\hline $\begin{array}{l}3,200 \\
3,200 \\
3,300 \\
3,120 \\
4,100\end{array}$ & $\begin{array}{l}4,710 \\
5,500 \\
4,200 \\
4,380 \\
5,500\end{array}$ & $\begin{array}{r}17 \\
31 \\
20 \\
19 \\
8\end{array}$ & $\begin{array}{l}2,040 \\
1,820 \\
1,860 \\
1,560 \\
2,040\end{array}$ & $\begin{array}{l}3,264 \\
3,500 \\
3,144 \\
3,600 \\
3,600\end{array}$ & $\begin{array}{l}18 \\
94 \\
36 \\
95 \\
32\end{array}$ & $\begin{array}{l}24 \frac{1}{3} \\
55 \\
32 \\
28 \frac{2}{3} \\
23\end{array}$ & $\begin{array}{l}16 \frac{1}{3} \\
99 \\
34 \\
84 \\
30\end{array}$ & $\begin{array}{c}40 \frac{2}{3} \\
154 \\
66 \\
112 \frac{2}{3} \\
53\end{array}$ & $\begin{array}{l}40 \\
39 \\
40 \\
36 \frac{1}{6} \\
40\end{array}$ & $\begin{array}{l}5 \\
5 \\
5 \frac{1}{2} \\
5 \\
6\end{array}$ & $\begin{array}{l}26,136 \\
31,289 \\
68,051 \\
14,000^{3} \\
30,710\end{array}$ & $\begin{array}{l}46 . \\
47 . \\
48 . \\
49 . \\
50 .\end{array}$ \\
\hline $\begin{array}{l}2,700 \\
3,000 \\
3,432 \\
2,400 \\
3,000\end{array}$ & $\begin{array}{l}3,400 \\
4,500 \\
5,412 \\
4,800 \\
3,200\end{array}$ & $\begin{array}{c}7 \\
16 \frac{1}{2} \\
14 \\
4 \\
7\end{array}$ & $\begin{array}{l}2,400 \\
2,080 \\
2,040 \\
2,100 \\
1,800\end{array}$ & $\begin{array}{l}2,700 \\
2,860 \\
3,600 \\
2,400 \\
2,500\end{array}$ & $\begin{array}{l}9 \\
28 \frac{1}{2} \\
40 \\
24 \\
18 \frac{1}{4}\end{array}$ & $\begin{array}{l}13 \frac{1}{2} \\
26 \frac{1}{2} \\
33 \\
17 \\
14\end{array}$ & \begin{tabular}{l}
\multicolumn{1}{c}{8} \\
$28 \frac{1}{2}$ \\
40 \\
24 \\
$18 \frac{1}{4}$
\end{tabular} & $\begin{array}{l}21 \frac{1}{2} \\
55 \\
73 \\
41 \\
32 \frac{1}{4}\end{array}$ & $\begin{array}{l}40 \\
35 \\
38 \frac{1}{2} \\
39 \frac{1}{2} \\
39\end{array}$ & $\begin{array}{l}5 \\
5 \\
{ }^{12} \\
5 \\
6\end{array}$ & $\begin{array}{l}\dddot{22}, 3^{12} \\
30,000 \\
20,061 \\
25,000^{9}\end{array}$ & $\begin{array}{l}51 . \\
52 . \\
53 . \\
54 . \\
55 .\end{array}$ \\
\hline $\begin{array}{l}2,760 \\
2,700 \\
3,500 \\
3,000 \\
3,324\end{array}$ & $\begin{array}{l}3,060 \\
3,660 \\
4,020 \\
4,300 \\
4,848\end{array}$ & $\begin{array}{c}12 \\
21 \\
18 \\
8 \\
19 \frac{2}{3}\end{array}$ & $\begin{array}{l}2,000 \\
1,200 \\
1,560 \\
1,800 \\
2,280\end{array}$ & $\begin{array}{l}2,400 \\
2,400 \\
3,360 \\
3,400 \\
3,168\end{array}$ & $\begin{array}{l}12 \\
28 \\
25 \\
29 \\
37\end{array}$ & $\begin{array}{l}23 \\
34 \frac{1}{4} \\
33 \\
17 \\
43 \frac{5}{6}\end{array}$ & $\begin{array}{l}11 \\
29 \frac{1}{2} \\
25 \\
28 \\
35\end{array}$ & $\begin{array}{l}34 \\
63^{\frac{3}{4}} \\
58 \\
45 \\
78 \frac{5}{8}\end{array}$ & $\begin{array}{l}39 \frac{1}{2} \\
38 \\
36 \frac{1}{3} \\
38 \frac{1}{2} \\
40\end{array}$ & $\begin{array}{l}5 \frac{1}{2} \\
6 \\
5 \\
5 \frac{1}{2} \\
5\end{array}$ & $\begin{array}{r}21,663 \\
26,879 \\
24,696 \\
11,692 \\
114,396\end{array}$ & $\begin{array}{l}56 . \\
57 . \\
58 . \\
59 . \\
60 .\end{array}$ \\
\hline $\begin{array}{l}3,200 \\
3,000 \\
3,312 \\
2,790 \\
3,120\end{array}$ & $\begin{array}{l}4,000 \\
4,000 \\
4,920 \\
3,720 \\
5,520\end{array}$ & $\begin{array}{r}9 \\
9 \\
20 \\
10 \\
34\end{array}$ & $\begin{array}{l}2,400 \\
1,680 \\
2,112 \\
1,800 \\
2,160\end{array}$ & $\begin{array}{l}3,000 \\
3,300 \\
3,600 \\
3,000 \\
4,200\end{array}$ & $\begin{array}{r}5 \\
6 \\
40 \\
30 \\
60\end{array}$ & $\begin{array}{l}19 \\
13 \\
29 \\
19 \frac{1}{3} \\
46 \frac{\frac{1}{4}}{4}\end{array}$ & $\begin{array}{c}25 \\
6 \\
40 \\
36 \\
70 \frac{1}{2}\end{array}$ & $\begin{array}{c}44 \\
19 \\
69 \\
55 \frac{1}{2} \\
117 \frac{1}{4}\end{array}$ & $\begin{array}{l}40 \\
37 \frac{1}{2} \\
40 \\
38 \\
40\end{array}$ & $\begin{array}{c}5 \\
5 \\
.^{12} \\
5 \\
5\end{array}$ & $\begin{array}{l}45,000 \\
8,962 \\
35,500 \\
35,184\end{array}$ & $\begin{array}{l}61 . \\
62 . \\
63 . \\
64 . \\
65 .\end{array}$ \\
\hline $\begin{array}{l}3,600 \\
4,530 \\
3,230 \\
3,432 \\
2,800\end{array}$ & $\begin{array}{l}5,760 \\
6,597 \\
5,000 \\
5,376 \\
4,200\end{array}$ & $\begin{array}{l}15 \\
18 \frac{1}{5} \\
13 \\
60 \\
77\end{array}$ & $\begin{array}{l}2,400 \\
3,043 \\
1,870 \\
2,172 \\
1,680\end{array}$ & $\begin{array}{l}3,840 \\
5,401 \\
3,230 \\
3,612 \\
3,000\end{array}$ & $\begin{array}{c}35 \\
41 \frac{1}{2} \\
32 \\
25 \\
115\end{array}$ & $\begin{array}{c}21 \\
26 \frac{3}{5} \\
22 \\
81 \\
103 \frac{1}{2}\end{array}$ & $\begin{array}{c}35 \\
34 \frac{5}{8} \\
30 \\
25 \\
132 \frac{1}{2}\end{array}$ & $\begin{array}{c}56 \\
61 \frac{1}{4} \\
52 \\
106 \\
236\end{array}$ & $\begin{array}{l}40 \\
40 \\
38 \frac{1}{2} \\
40 \\
37 \frac{1}{2}\end{array}$ & $\begin{array}{l}5 \\
5 \\
5 \frac{1}{2} \\
5 \\
5\end{array}$ & $\begin{array}{l}37,314 \\
61,369 \\
35,398 \\
83,044 \\
\ldots \ldots \ldots\end{array}$ & $\begin{array}{l}66 . \\
67 . \\
68 . \\
69 . \\
70 .\end{array}$ \\
\hline $\begin{array}{r}4,530 \\
3,200 \\
1,800 \\
70\end{array}$ & $\begin{array}{r}9,500 \\
4,470 \\
3,000 \\
70\end{array}$ & $\begin{array}{c}111 \frac{1}{3} \\
16 \frac{3}{4} \\
2 \\
70\end{array}$ & $\begin{array}{r}3,043 \\
1,968 \\
1,200 \\
70\end{array}$ & $\begin{array}{r}6,300 \\
3,247 \\
2,196 \\
70\end{array}$ & $\begin{array}{c}220 \frac{2}{3} \\
27 \frac{1}{2} \\
40 \\
70\end{array}$ & $\begin{array}{l}137 \frac{1}{3} \\
27 \frac{3}{10} \\
6 \\
70\end{array}$ & $\begin{array}{c}227 \\
27 \frac{5}{8} \\
6 \\
70\end{array}$ & $\begin{array}{l}358 \\
55 \frac{1}{8} \\
12 \\
70\end{array}$ & $\begin{array}{l}41 \frac{1}{2} \\
39 \\
35 \\
70\end{array}$ & $\begin{array}{r}6 \\
5 \\
5 \\
66\end{array}$ & $\begin{array}{c}191,567 \\
30,690 \mathrm{~N} \\
7,500 \\
64\end{array}$ & $\begin{array}{l}\text { High } \\
\text { Iedian } \\
\text { Low } \\
\mathrm{N}^{14}\end{array}$ \\
\hline
\end{tabular}

mate. 10 Knoxville campus only. 11 Includes student assistants. 12 Not reported or not available. 13 Curator of oriental collections. 14 Number of libraries upon which high, median, and low are based. 15 Acting director. 16 Includes all hourly help. 17 Ten months only. 18 Included with depart- 


\begin{tabular}{|c|c|c|c|c|c|c|c|}
\hline Library & $\begin{array}{c}\text { Fiscal } \\
\text { Year } \\
\text { Ending }\end{array}$ & $\begin{array}{l}\text { Student } \\
\text { Total } \\
\text { Under- } \\
\text { graduates }\end{array}$ & $\begin{array}{l}\text { Trollment } \\
\text { Total } \\
\text { Graduates }\end{array}$ & $\begin{array}{l}\text { Book } \\
\text { Stock }\end{array}$ & $\begin{array}{l}\text { Volumes } \\
\text { Added }\end{array}$ & $\begin{array}{l}\text { News- } \\
\text { papers } \\
\text { Currently } \\
\text { Received }\end{array}$ & $\begin{array}{c}\text { Periodicals } \\
\text { Currently } \\
\text { Received }\end{array}$ \\
\hline 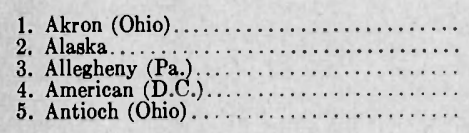 & $\begin{array}{l}\text { 31De53 } \\
\text { 30Je54 } \\
\text { 30Je54 } \\
\text { 30Je54 } \\
\text { 30Je54 }\end{array}$ & $\begin{array}{r}3,896^{1} \\
345 \\
930 \\
3,419 \\
999\end{array}$ & $\begin{array}{r}46 \\
14 \\
7 \\
2,305\end{array}$ & $\begin{array}{r}96,168 \\
36,719 \\
131,666 \\
161,726 \\
81,567\end{array}$ & $\begin{array}{l}3,505^{\mathrm{a}} \\
1,867^{\mathrm{k}} \\
3,289^{\mathrm{a}} \\
1,447^{\mathrm{e}} \\
2,021^{\mathrm{a}}\end{array}$ & $\begin{array}{r}11 \\
11 \\
9 \\
7 \\
9\end{array}$ & $\begin{array}{l}592^{1} \\
563^{j} \\
5081 \\
570^{1} \\
544^{j}\end{array}$ \\
\hline 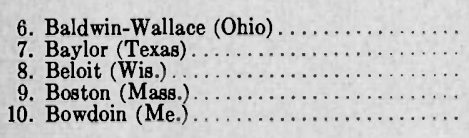 & $\begin{array}{r}\text { 30Je54 } \\
\text { 31 Ag54 } \\
\text { 30Je54 } \\
\text { 30Je54 } \\
\text { 30Je54 }\end{array}$ & $\begin{array}{r}1,277 \\
4,406 \\
860 \\
4,462 \\
768\end{array}$ & $\begin{array}{r}\dddot{421} \\
3 \\
1,318 \\
\cdots\end{array}$ & $\begin{array}{r}58,357 \\
226,677 \\
181,129 \\
429,978 \\
234,492\end{array}$ & $\begin{array}{l}2,288^{\mathrm{h}} \\
9,145^{\mathrm{b}} \\
1,475^{\mathrm{e}} \\
7,577^{\mathrm{e}} \\
4,416^{\mathrm{a}}\end{array}$ & $\begin{array}{r}7 \\
43 \\
12 \\
20 \\
11\end{array}$ & $\begin{array}{r}496^{\mathrm{j}} \\
1,161^{\mathrm{j}} \\
510^{\mathrm{j}} \\
1,476^{\mathrm{j}} \\
507^{\mathrm{j}}\end{array}$ \\
\hline 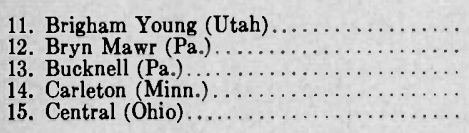 & $\begin{array}{l}31 \mathrm{Ag} 54 \\
\text { 30Je54 } \\
\text { 30Je54 } \\
\text { 30Je54 } \\
30 \mathrm{Ap54}\end{array}$ & $\begin{array}{r}6,347 \\
607 \\
1,846 \\
860 \\
820\end{array}$ & $\begin{array}{r}243 \\
148 \\
26 \\
\cdots \\
\cdots\end{array}$ & $\begin{array}{r}218,199 \\
231,061 \\
138,719 \\
148,336 \\
28,103\end{array}$ & $\begin{array}{l}9,664^{\mathrm{a}} \\
6,175^{\mathrm{a}} \\
5,894^{\mathrm{a}} \\
2,603^{\mathrm{b}} \\
2,435^{\mathrm{g}}\end{array}$ & $\begin{array}{r}37 \\
8 \\
13 \\
19 \\
21\end{array}$ & $\begin{array}{l}887 \mathfrak{j} \\
846^{j} \\
975^{j} \\
483^{j} \\
337 j\end{array}$ \\
\hline 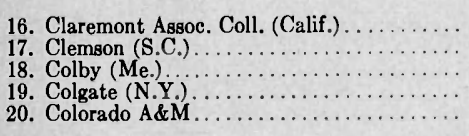 & $\begin{array}{l}\text { 1Je54 } \\
\text { 30Je54 } \\
\text { 30Je54 } \\
\text { 30Je54 } \\
\text { 30Je54 }\end{array}$ & $\begin{array}{l}1,700 \\
2,685 \\
1,009 \\
1,329 \\
3,365\end{array}$ & $\begin{array}{r}200 \\
51 \\
\cdots 33 \\
180\end{array}$ & $\begin{array}{l}220,000 \\
141,281 \\
161,928 \\
211,020 \\
169,070\end{array}$ & $\begin{array}{l}9,900^{\mathbf{k}} \\
7,116^{\mathrm{a}} \\
4,637^{\mathrm{g}} \\
3,526^{\mathrm{d}} \\
4,764^{\mathrm{d}}\end{array}$ & $\begin{array}{r}20 \\
43 \\
5 \\
24 \\
8\end{array}$ & $\begin{array}{r}1,299^{\mathrm{j}} \\
996^{\mathrm{j}} \\
581^{\mathrm{j}} \\
562^{\mathrm{j}} \\
1,200^{\mathrm{j}}\end{array}$ \\
\hline 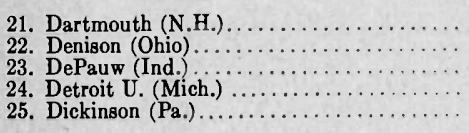 & $\begin{array}{l}\text { 30Je54 } \\
\text { 31Jy54 } \\
\text { 30Je54 } \\
\text { 30Je54 } \\
\text { 30Je54 }\end{array}$ & $\begin{array}{l}2,653 \\
1,236 \\
1,722 \\
7,668 \\
868\end{array}$ & $\begin{array}{r}204 \\
17 \\
519\end{array}$ & $\begin{array}{r}727,467 \\
104,199 \\
124,830 \\
166,095 \\
97,646\end{array}$ & $\begin{array}{r}15,096^{\mathrm{a}} \\
3,453^{\mathrm{a}} \\
3,622^{\mathrm{a}} \\
6,841^{\mathrm{h}} \\
2,412^{\mathrm{h}}\end{array}$ & $\begin{array}{l}47 \\
18 \\
16 \\
10 \\
16\end{array}$ & $\begin{array}{r}2,492^{\mathrm{j}} \\
538^{\mathrm{j}} \\
521^{\mathrm{j}} \\
1,227^{\mathrm{j}} \\
398^{\mathrm{j}}\end{array}$ \\
\hline $\begin{array}{l}\text { 26. Duquesne }(\mathrm{Pa} .) \ldots \ldots \ldots \\
\text { 27. Hamilton (N.Y.) } \ldots \ldots \ldots \\
\text { 28. Holy Cross (Mass.) } \ldots . . \\
\text { 29. Houston (Texas) } \ldots \ldots \ldots \\
\text { 30. Howard (Ala. } \ldots \ldots \ldots \ldots\end{array}$ & $\begin{array}{r}31 \mathrm{My} 54 \\
30 \mathrm{Je54} \\
31 \mathrm{My} 54 \\
31 \mathrm{Ag} 54 \\
31 \mathrm{Ag} 54\end{array}$ & $\begin{array}{r}3,333 \\
584 \\
1,830 \\
8,787 \\
1,972\end{array}$ & $\begin{array}{r}352 \\
7 \\
1,545\end{array}$ & $\begin{array}{r}61,517 \\
233,746 \\
163,456 \\
128,389 \\
54,708\end{array}$ & $\begin{array}{r}4,702^{\mathrm{a}} \\
4,778^{\mathrm{h}} \\
5,192^{\mathrm{k}} \\
16,081^{\mathrm{a}} \\
2,695^{\mathrm{b}}\end{array}$ & $\begin{array}{r}7 \\
8 \\
27 \\
16 \\
28\end{array}$ & $\begin{array}{r}942^{\mathrm{j}} \\
551^{\mathrm{j}} \\
341^{\mathrm{j}} \\
1,724 \mathrm{j} \\
650^{\mathrm{j}}\end{array}$ \\
\hline 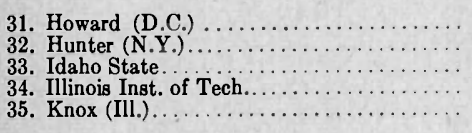 & $\begin{array}{l}\text { 30Je54 } \\
\text { 30Je54 } \\
\text { 30Je54 } \\
31 \mathrm{Ag} 54 \\
\text { 30Je54 }\end{array}$ & $\begin{array}{r}2,557 \\
11,338 \\
1,263 \\
5,271 \\
775\end{array}$ & $\begin{array}{r}887 \\
903 \\
1,038\end{array}$ & $\begin{array}{r}300,377 \\
186,187 \\
45,481 \\
123,867 \\
79,933\end{array}$ & $\begin{array}{l}9,645^{\mathrm{e}} \\
8,170^{\mathrm{b}} \\
3,960^{\mathrm{e}} \\
2,026^{\mathrm{c}} \\
3,025^{\mathrm{a}}\end{array}$ & $\begin{array}{r}20 \\
5 \\
57 \\
3 \\
10\end{array}$ & $\begin{array}{r}1,583 \\
630^{\mathrm{J}} \\
797 \mathrm{j} \\
922^{1} \\
325^{\mathrm{J}}\end{array}$ \\
\hline 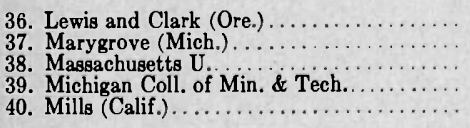 & $\begin{array}{r}\text { 31 My54 } \\
\text { 30Je54 } \\
\text { 30Je54 } \\
\text { 30Je54 } \\
\text { 30Je54 }\end{array}$ & $\begin{array}{r}969 \\
660 \\
3,504 \\
1,499 \\
497\end{array}$ & $\begin{array}{r}21 \\
310 \\
19 \\
60\end{array}$ & $\begin{array}{r}39,176 \\
68,663 \\
189,670 \\
62,921 \\
108,806\end{array}$ & $\begin{array}{l}3,364^{\mathrm{a}} \\
2,970^{\mathrm{a}} \\
7,094^{\mathrm{b}} \\
1,425^{\mathrm{a}} \\
1,639^{\mathrm{h}}\end{array}$ & $\begin{array}{r}29 \\
12 \\
5 \\
26 \\
3\end{array}$ & $\begin{array}{l}459 j \\
459^{j} \\
834^{1} \\
6041 \\
459^{i}\end{array}$ \\
\hline 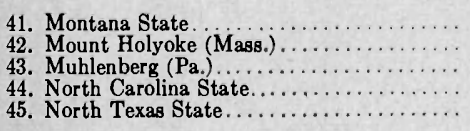 & $\begin{array}{r}\text { 30Je54 } \\
\text { 30Je54 } \\
31 \mathrm{My} 54 \\
30 \mathrm{Je54} \\
31 \mathrm{Ag} 54\end{array}$ & $\begin{array}{r}1,891 \\
1,185 \\
676 \\
3,758 \\
4,219\end{array}$ & $\begin{array}{r}88 \\
45 \\
\ldots \\
297 \\
357\end{array}$ & $\begin{array}{r}97,443 \\
240,152 \\
87,125 \\
139,018 \\
259,974\end{array}$ & $\begin{array}{c}4,071^{\mathrm{g}} \\
5,171^{\mathrm{a}} \\
2,750^{\mathrm{a}} \\
11,542^{\mathrm{c}} \\
25,009^{\mathrm{a}, 2}\end{array}$ & $\begin{array}{l}32 \\
13 \\
11 \\
38 \\
40\end{array}$ & $\begin{array}{r}1,786^{1} \\
725 \mathrm{k} \\
415 \mathrm{j} \\
1,776^{\mathrm{i}} \\
1,284 \mathrm{j}\end{array}$ \\
\hline 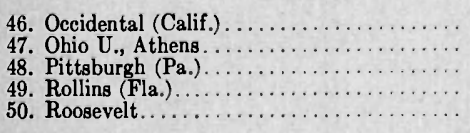 & $\begin{array}{l}\text { 30Je54 } \\
\text { 30Je54 } \\
\text { 30Je54 } \\
\text { 30Je54 } \\
31 \mathrm{Ag} 54\end{array}$ & $\begin{array}{r}1,259 \\
4,304 \\
11,529 \\
629 \\
2,822\end{array}$ & $\begin{array}{r}76 \\
154 \\
3,562 \\
224\end{array}$ & $\begin{array}{l}113,271 \\
233,479 \\
684,229 \\
88,437 \\
91,321\end{array}$ & $\begin{array}{r}4,172^{\mathrm{a}} \\
7,492^{\mathrm{d}} \\
23,809^{\mathrm{e}} \\
3,434^{\mathrm{g}} \\
9,102^{\mathrm{c}}\end{array}$ & $\begin{array}{r}16 \\
14 \\
6 \\
15 \\
25\end{array}$ & $\begin{array}{r}679 j \\
1,0981 \\
1,592 \mathrm{j} \\
357 \mathrm{j} \\
659 \mathrm{j}\end{array}$ \\
\hline 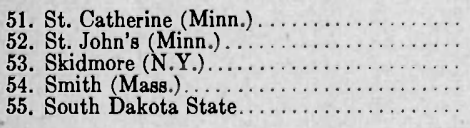 & $\begin{array}{l}\text { 30Je54 } \\
\text { 30Je54 } \\
\text { 30Je54 } \\
\text { 30Je54 } \\
\text { 30Je54 }\end{array}$ & $\begin{array}{r}854 \\
813 \\
1,004 \\
2,169 \\
1,822\end{array}$ & $\begin{array}{l}113 \\
73 \\
51\end{array}$ & $\begin{array}{r}80,605 \\
92,990 \\
73,811 \\
394,209 \\
106,389\end{array}$ & $\begin{array}{l}2,199^{\mathrm{e}} \\
3,111^{\mathrm{a}} \\
2,304^{\mathrm{b}} \\
9,435^{\mathrm{a}} \\
3,058^{\mathrm{a}}\end{array}$ & $\begin{array}{r}18 \\
21 \\
7 \\
31 \\
14\end{array}$ & $\begin{array}{r}464 j \\
540^{k} \\
549 j \\
1,313 j \\
1,040 j\end{array}$ \\
\hline 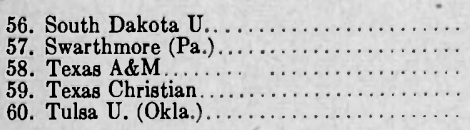 & $\begin{array}{r}\text { 30Je54 } \\
\text { 30Je54 } \\
31 \mathrm{Ag} 54 \\
31 \mathrm{Ag} 54 \\
31 \mathrm{My} 54\end{array}$ & $\begin{array}{r}1,340 \\
913 \\
5,783 \\
3,562 \\
2,112\end{array}$ & $\begin{array}{r}85 \\
6 \\
415 \\
340 \\
134\end{array}$ & $\begin{array}{l}148,198 \\
172,502 \\
258,962 \\
232,706 \\
155,791\end{array}$ & $\begin{array}{r}4,100^{\mathrm{b}} \\
5,680^{\mathrm{a}} \\
19,222^{\mathrm{a}} \\
15,907^{\mathrm{a}} \\
6,993^{\mathrm{a}}\end{array}$ & $\begin{array}{l}13 \\
11 \\
49 \\
14 \\
17\end{array}$ & $\begin{array}{r}1,389 \mathrm{j} \\
741^{\mathrm{j}} \\
3,507^{1} \\
\mathbf{7 7 0 j} \\
574^{j}\end{array}$ \\
\hline 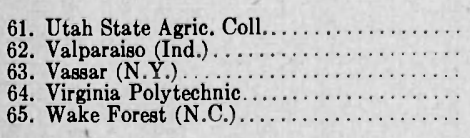 & $\begin{array}{l}\text { 30Je54 } \\
\text { 30Je54 } \\
\text { 30Je54 } \\
\text { 30Je54 } \\
\text { 30Je54 }\end{array}$ & $\begin{array}{l}2,790 \\
1,790 \\
1,414 \\
2,966 \\
1,662\end{array}$ & $\begin{array}{r}267 \\
13 \\
199 \\
7\end{array}$ & $\begin{array}{r}171,875 \\
75,069 \\
296,774 \\
183,055 \\
129,632\end{array}$ & $\begin{array}{r}5,800^{\mathrm{k}} \\
4,016^{\mathrm{b}} \\
8,403^{\mathrm{a}} \\
24,388^{\mathrm{g}} \\
6,245^{\mathrm{b}}\end{array}$ & $\begin{array}{l}85 \\
12 \\
26 \\
91 \\
36\end{array}$ & $\begin{array}{r}1,931^{1} \\
448^{j} \\
1,200 k \\
1,775^{k} \\
1,091^{j}\end{array}$ \\
\hline 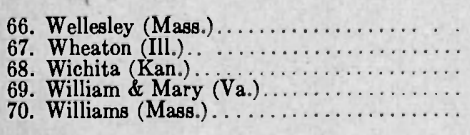 & $\begin{array}{c}\text { 30Je54 } \\
\text { 31^ g54 } \\
\text { 30Je54 } \\
\text { 30Je54 } \\
\text { 30Je54 }\end{array}$ & $\begin{array}{l}1,690 \\
1,575 \\
3,069 \\
1,635 \\
1,063\end{array}$ & $\begin{array}{r}28 \\
91 \\
250 \\
12 \\
13\end{array}$ & $\begin{array}{l}294,331 \\
100,942 \\
111,212 \\
237,594 \\
219,012\end{array}$ & $\begin{array}{l}6,116^{\mathrm{b}} \\
1,949^{\mathrm{a}} \\
7,743^{\mathrm{d}} \\
6,235^{\mathrm{b}} \\
3,610^{\mathrm{b}}\end{array}$ & $\begin{array}{r}20 \\
6 \\
9 \\
30 \\
9\end{array}$ & $\begin{array}{r}1,124 ! \\
5444^{\mathrm{j}} \\
816 \\
1,351^{\mathrm{j}} \\
763^{\mathrm{j}}\end{array}$ \\
\hline & $\begin{array}{l}\ldots \ldots \ldots \\
\ldots \ldots \ldots \\
\ldots \ldots \ldots\end{array}$ & $\begin{array}{r}11,529 \\
1,695 \\
345 \\
69\end{array}$ & $\begin{array}{r}3,562 \\
124 \\
3 \\
52\end{array}$ & $\begin{array}{r}727,467 \\
148,267 \\
28,103 \\
70\end{array}$ & $\begin{array}{r}24,388 \\
4,637 \\
1,425 \\
69\end{array}$ & $\begin{array}{l}91 \\
5.5 \\
3 \\
70\end{array}$ & $\begin{array}{r}3,507 \\
733 \\
325 \\
70\end{array}$ \\
\hline
\end{tabular}

1 No breakdown for 1,828 evening students included here. 212,801 documents included which were not previously reported. 3 Includes student

service. 4 Number of libraries on which high, median and low are based.
a Physical count, includes documents. b Physical count, does not include documents. c Physical count only reported. d Bibliographical count, in- 


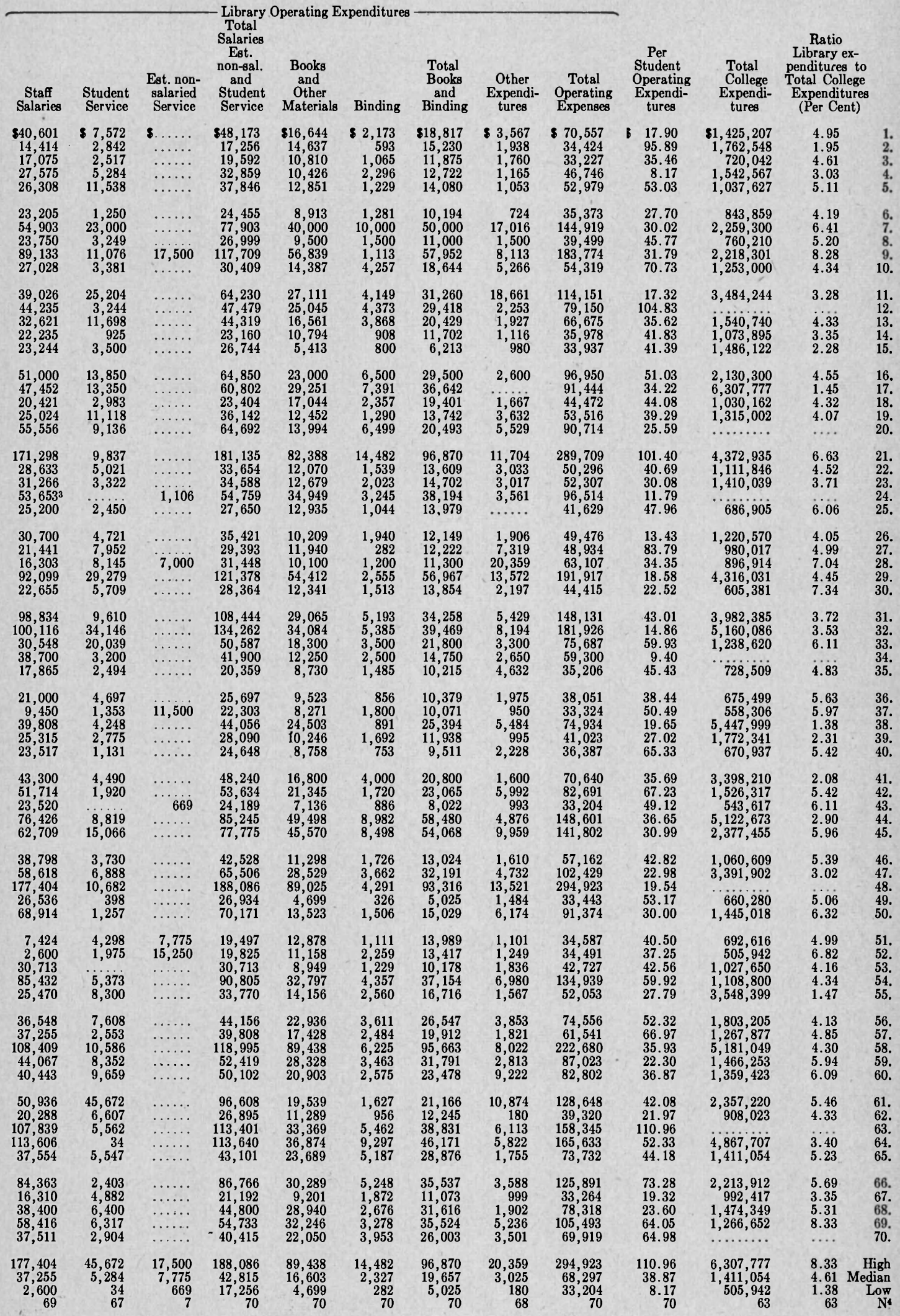

cludes documents. e Bibliographical count, does not include documents. \& Bibliographical count only reported. g Documents included, no physical or bibliographical count reported. h Documents not included, no physical or bibliographical count reported. I Includes non-periodical serials. $\mathbf{j}$ Does not include non-periodical serials. $k$ Information $(a-j)$ not reported. 


\section{College and University Library Budget}

Budget, 1954-1955

Salaries September 1, 1954

\begin{tabular}{|c|c|c|c|c|c|c|c|c|}
\hline Library & $\begin{array}{c}\text { Salaries } \\
\text { and } \\
\text { Wages }\end{array}$ & $\begin{array}{l}\text { Books } \\
\text { Periodi- } \\
\text { cals \& } \\
\text { Binding }\end{array}$ & $\begin{array}{c}\text { Total } \\
\text { Library } \\
\text { Operating } \\
\text { Budget }\end{array}$ & $\begin{array}{l}\text { Chief } \\
\text { Librarian }\end{array}$ & $\begin{array}{l}\text { Associate } \\
\text { Chief } \\
\text { Librarian }\end{array}$ & $\begin{array}{l}\text { Depa } \\
\text { Minimum }\end{array}$ & $\begin{array}{l}\text { rtment Head } \\
\text { Maximum }\end{array}$ & No. \\
\hline 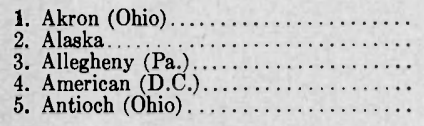 & $\begin{array}{l}\$ 51,920 \\
17,178 \\
22,539 \\
29,600 \\
38,800\end{array}$ & $\begin{array}{r}\$ 21,000 \\
15,822 \\
14,892 \\
9,800 \\
9,800\end{array}$ & $\begin{array}{l}76,777 \\
33,800 \\
39,032 \\
39,400 \\
50,450\end{array}$ & $\begin{array}{r}\$ 7,500 \\
5,200 \\
6,650\end{array}$ & $\begin{array}{l}\ldots \ldots \\
\cdots \cdots \\
4,200 \\
\cdots \cdots\end{array}$ & $\begin{array}{r}\ldots \ldots \\
\cdots \cdots \\
\$ 3,300 \\
3,600\end{array}$ & $\begin{array}{c}\ldots \ldots \\
\ldots \ldots \\
\ldots \ldots \\
\$ 5,000\end{array}$ & $\begin{array}{l}\cdots \\
\cdots\end{array}$ \\
\hline $\begin{array}{r}\text { 6. Baldwin-Wallace (Ohio) } \ldots \ldots \ldots \ldots \ldots \ldots \\
\text { 7. Baylor (Texas) } \ldots \ldots \ldots \ldots \ldots \ldots \ldots \ldots \ldots \ldots \\
\text { 8. Beloit (Wis.) } \ldots \ldots \ldots \ldots \ldots \ldots \ldots \ldots \ldots \ldots \ldots \\
\text { 9. Boston (Mass.) } \ldots \ldots \ldots \ldots \ldots \ldots \ldots \ldots \ldots \\
\text { 10. Bowdoin (Me.) } \ldots \ldots \ldots \ldots \ldots \ldots\end{array}$ & $\begin{array}{r}24,000 \\
77,903 \\
27,300 \\
125,000 \\
32,160\end{array}$ & $\begin{array}{r}8,000 \\
50,000 \\
10,500 \\
60,000 \\
19,000\end{array}$ & $\begin{array}{r}32,700 \\
144,919 \\
39,300 \\
195,000 \\
53,660\end{array}$ & $\begin{array}{l}\cdots \\
5,500 \\
7,500 \\
7,780\end{array}$ & $\begin{array}{l}6,300 \\
4,400 \\
4,980\end{array}$ & $\begin{array}{l}3,400 \\
3,700 \\
3,600\end{array}$ & $\begin{array}{l}4,100 \\
\ldots \ldots \\
\cdots \cdots \\
3 \ldots \\
3,800\end{array}$ & $\begin{array}{l}3 \\
2 \\
2 \\
2\end{array}$ \\
\hline 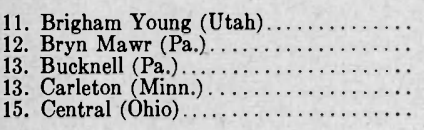 & $\begin{array}{l}93,019 \\
51,800 \\
45,400 \\
23,775 \\
27,944\end{array}$ & $\begin{array}{r}73,500 \\
24,850 \\
22,000 \\
12,090 \\
9,000\end{array}$ & $\begin{array}{r}178,329 \\
79,650 \\
69,825 \\
37,975 \\
38,944\end{array}$ & $\begin{array}{l}5,500 \\
5,600 \\
5,200\end{array}$ & $\begin{array}{l}4,900 \\
\cdots \cdots \\
\cdots \cdots \\
\cdots \cdots\end{array}$ & $\begin{array}{l}3,600 \\
3,250 \\
\dddot{3}, 635\end{array}$ & $\begin{array}{l}4,350 \\
3,800 \\
3,440\end{array}$ & $\begin{array}{l}4 \\
\ddot{4} \\
\ddot{5}\end{array}$ \\
\hline $\begin{array}{l}\text { 16. Claremont Assoc. Coll. (Calif.) } \ldots \ldots \ldots \\
\text { 17. Clemson (S.C.) } \ldots \ldots \ldots \ldots \ldots \ldots \ldots \ldots \ldots \\
\text { 18. Colby (Me.) } \ldots \ldots \ldots \ldots \ldots \ldots \ldots \ldots \ldots \\
\text { 19. Colgate (N.Y.) } \\
\text { 20. Colorado A \& }{ }_{\text {M. }} \ldots \ldots \ldots \ldots \ldots \ldots \ldots \ldots\end{array}$ & $\begin{array}{l}71,552 \\
66,978 \\
31,700 \\
40,705 \\
66,246\end{array}$ & $\begin{array}{l}30,000 \\
33,718 \\
18,300 \\
13,600 \\
26,260^{\text { }}\end{array}$ & $\begin{array}{r}110,352 \\
105,708 \\
51,900 \\
58,989 \\
92,506\end{array}$ & \begin{tabular}{l}
6,600 \\
6,500 \\
6,000 \\
\hdashline$\dddot{6}, 350$
\end{tabular} & $\begin{array}{l}4,860 \\
3,800 \\
4,300 \\
5,000\end{array}$ & $\begin{array}{l}3,600 \\
3,600 \\
3,600 \\
4,475\end{array}$ & $\begin{array}{l}3,620 \\
4,435 \\
4,300 \\
\cdots\end{array}$ & $\begin{array}{l}5 \\
1 \\
3 \\
1\end{array}$ \\
\hline $\begin{array}{l}\text { 21. Dartmouth (N.H.) } \ldots \ldots \ldots \ldots \ldots \ldots \ldots \\
\text { 22. Denison (Ohio) } \ldots \ldots \ldots \ldots \ldots \ldots \ldots \ldots \\
\text { 23. DePauw (Ind.) } \\
\text { 24. Detroit U. (Mich.) } \ldots \ldots \ldots \ldots \ldots \ldots \ldots \ldots \ldots \\
\text { 25. Dickinson }(\mathrm{Pa} .) \ldots \ldots \ldots \ldots \ldots \ldots \ldots\end{array}$ & $\begin{array}{r}199,800 \\
35,025 \\
38,618 \\
82,277 \\
25,600\end{array}$ & $\begin{array}{r}87,475 \\
14,800 \\
15,884 \\
48,780 \\
9,000\end{array}$ & $\begin{array}{r}299,300 \\
52,725 \\
57,244 \\
138,057 \\
35,100\end{array}$ & $\begin{array}{l}5,000 \\
\ldots \ldots \\
5,000\end{array}$ & $\begin{array}{l}\ldots \ldots \\
\ldots \ldots \\
5,000 \\
\ldots \ldots\end{array}$ & $\begin{array}{l}2,400 \\
3,800 \\
4,500 \\
\cdots\end{array}$ & $\begin{array}{l}4,800 \\
\cdots \cdots \\
6,500 \\
\cdots \cdots\end{array}$ & $\begin{array}{r}13 \\
1 \\
.3 \\
. .\end{array}$ \\
\hline $\begin{array}{l}\text { 26. Duquesne (Pa.) } \ldots \ldots \ldots \ldots \ldots \ldots \ldots \ldots \\
\text { 27. Hamilton (N.Y.) } \ldots \ldots \ldots \ldots \ldots \ldots \ldots \ldots \\
\text { 28. Holy Cross (Mass.) } \ldots \ldots \ldots \ldots \ldots \ldots \ldots \ldots \\
\text { 29. Houston (Texas) } \ldots \ldots \ldots \ldots \ldots \ldots \ldots \ldots \\
\text { 30. Howard (Ala.) } \ldots \ldots \ldots \ldots \ldots \ldots \ldots\end{array}$ & $\begin{array}{r}37,981 \\
30,400 \\
31,500 \\
126,500 \\
26,962\end{array}$ & $\begin{array}{l}20,580 \\
14,000 \\
12,000 \\
60,000 \\
10,200\end{array}$ & $\begin{array}{r}60,581 \\
49,150 \\
63,500 \\
201,500 \\
39,562\end{array}$ & $\begin{array}{l}5,000 \\
7,000 \\
8,040 \\
5,100\end{array}$ & $\begin{array}{l}\ldots{ }^{28} \\
\ldots \ldots \\
\cdots{ }^{29} \\
3,900\end{array}$ & $\begin{array}{l}3,300 \\
3,200 \\
4,200^{14} \\
3,300\end{array}$ & $\begin{array}{l}\cdots \\
4,000 \\
4,885^{14} \\
\cdots\end{array}$ & $\begin{array}{l}3 \\
3 \\
7 \\
1\end{array}$ \\
\hline 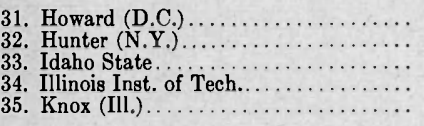 & $\begin{array}{r}101,019 \\
135,000 \\
47,135 \\
42,920 \\
20,000\end{array}$ & $\begin{array}{l}38,029 \\
40,000 \\
18,550 \\
15,200 \\
11,000\end{array}$ & $\begin{array}{r}143,782 \\
183,000 \\
70,485 \\
60,720 \\
36,000\end{array}$ & $\begin{array}{l}6,970 \\
7,900 \\
5,700 \\
7,500 \\
5,750\end{array}$ & $\begin{array}{l}7,618 \\
4,160 \\
\ldots \ldots \\
\ldots \ldots\end{array}$ & $\begin{array}{l}4,163 \\
5,374 \\
3,700 \\
3,000\end{array}$ & $\begin{array}{l}5,009 \\
5,938 \\
3,480 \\
4,850 \\
4,200\end{array}$ & $\begin{array}{l}4 \\
3 \\
1 \\
2 \\
2\end{array}$ \\
\hline $\begin{array}{l}\text { 36. Lewis and Clark (Ore.) } \ldots \ldots \ldots \ldots \ldots \ldots \\
\text { 37. Marygrove (Mich.) } \ldots \ldots \ldots \ldots \ldots \ldots \ldots \\
\text { 38. Massachusetts U.. } \ldots \ldots \ldots \ldots \ldots \ldots \\
\text { 39. Michigan Coll. of Min. \& Tech } \ldots \ldots \ldots \ldots \\
\text { 40. Mills (Calif.) } \ldots \ldots \ldots \ldots \ldots \ldots \ldots \ldots\end{array}$ & $\begin{array}{l}25,000^{1} \\
23,125 \\
57,170 \\
30,430 \\
23,517\end{array}$ & $\begin{array}{r}9,460 \\
11,000 \\
25,175 \\
14,310 \\
5,000\end{array}$ & $\begin{array}{l}36,460^{1} \\
35,125 \\
85,675 \\
45,951 \\
28,917\end{array}$ & $\begin{array}{l}5,400^{2} \\
4,000 \\
6,760 \\
5,295 \\
6,200\end{array}$ & $\begin{array}{l}3,900 \\
3,000 \\
\cdots \cdots \\
\cdots \cdots\end{array}$ & $\begin{array}{l}3,000 \\
3,000 \\
\cdots \cdots \\
\cdots \cdots\end{array}$ & $\begin{array}{l}3,700 \\
\cdots \cdots \\
\cdots \cdots \\
\cdots \cdots\end{array}$ & $\begin{array}{r}3 \\
3 \\
\cdots \\
\cdots \\
\cdots\end{array}$ \\
\hline $\begin{array}{l}\text { 41. Montana State } \\
\text { 42. Mount Holyoke (Mass.) } \ldots \ldots \ldots \ldots \ldots \ldots \\
\text { 43. Muhlenberg }(\mathrm{Pa} .) \ldots \ldots \ldots \ldots \ldots \ldots \\
\text { 44. North Carolina State } \ldots \ldots \ldots \ldots \ldots \ldots \\
\text { 45. North Texas State } \ldots \ldots \ldots \ldots \ldots \ldots\end{array}$ & $\begin{array}{l}51,720 \\
56,100 \\
23,440 \\
89,785 \\
87,800^{3}\end{array}$ & $\begin{array}{r}22,500 \\
22,500 \\
9,000 \\
79,000 \\
49,000^{3}\end{array}$ & $\begin{array}{r}76,480 \\
81,810 \\
33,840 \\
174,535 \\
147,800\end{array}$ & $\begin{array}{l}6,000 \\
\ldots, 000 \\
7,333\end{array}$ & $\begin{array}{l}5,100 \\
4,500 \\
\therefore, 200\end{array}$ & $\begin{array}{l}4,350 \\
3,400 \\
\dddot{3}, 685\end{array}$ & $\begin{array}{l}4,450 \\
\ldots \ldots \\
\ldots \ldots \\
4,800\end{array}$ & $\begin{array}{l}2 \\
\cdots \\
\cdots \\
6\end{array}$ \\
\hline 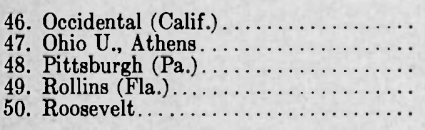 & $\begin{array}{r}46,135 \\
68,060 \\
188,086 \\
26,852 \\
79,580\end{array}$ & $\begin{array}{r}10,852 \\
35,600 \\
93,315 \\
5,350 \\
19,900\end{array}$ & $\begin{array}{r}58,962 \\
105,360 \\
294,922 \\
33,547 \\
108,780\end{array}$ & $\begin{array}{l}4,800 \\
7,100 \\
\dddot{4}, 500 \\
5,000\end{array}$ & $\begin{array}{l}\cdots \cdots \\
\cdots \cdots \\
\cdots \cdots \\
\cdots \cdots\end{array}$ & $\begin{array}{l}3,500 \\
3,680 \\
3,000 \\
3,850\end{array}$ & $\begin{array}{l}4,400 \\
\ldots \ldots \\
3,200 \\
4,450\end{array}$ & $\begin{array}{r}5 \\
4 \\
4 \\
4\end{array}$ \\
\hline 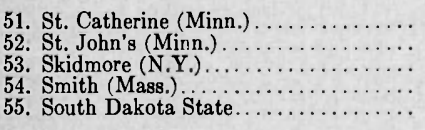 & $\begin{array}{l}20,000 \\
19,350 \\
33,321 \\
95,300 \\
33,200\end{array}$ & $\begin{array}{l}15,000 \\
11,500 \\
10,650 \\
49,219 \\
11,300\end{array}$ & $\begin{array}{r}36,000 \\
32,425 \\
45,971 \\
161,994 \\
45,700\end{array}$ & $\begin{array}{l}5,200 \\
6,000 \\
4,650 \\
6,100\end{array}$ & $\begin{array}{l}\ddot{3}, \dot{500} \\
\cdots \cdots \\
\cdots \cdots \\
\cdots \cdots\end{array}$ & $\begin{array}{l}3,500 \\
\cdots \cdots \\
3,500 \\
4,000\end{array}$ & $\begin{array}{l}5,200 \\
\cdots \cdots \\
4,400 \\
4,100\end{array}$ & $\begin{array}{l}\cdots \\
\cdots \\
6 \\
3\end{array}$ \\
\hline 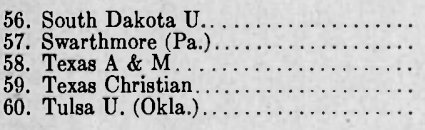 & $\begin{array}{r}44,852 \\
41,500 \\
142,050 \\
47,595 \\
48,909\end{array}$ & $\begin{array}{l}29,550 \\
20,800 \\
85,000 \\
34,000 \\
24,350\end{array}$ & $\begin{array}{r}77,992 \\
64,500 \\
236,050 \\
84,845 \\
79,266\end{array}$ & $\begin{array}{l}5,000 \\
7,500 \\
5,000 \\
6,000\end{array}$ & $\begin{array}{l}\cdots \\
5,870 \\
\cdots \cdots\end{array}$ & $\begin{array}{l}3,400 \\
4,160 \\
3,600 \\
3,600\end{array}$ & $\begin{array}{l}4,500 \\
\because, 380 \\
4,364 \\
4,400\end{array}$ & $\begin{array}{l}2 \\
2 \\
4 \\
3 \\
3\end{array}$ \\
\hline $\begin{array}{l}\text { 61. Utah State Agric. Coll..... } \ldots \ldots \ldots \ldots \\
\text { 62. Valparaiso (Ind.) } \ldots \ldots \ldots \ldots \ldots \ldots \ldots \\
\text { 63. Vassar (N.Y.) } \\
\text { 64. Virginia Polytechnic } \ldots \ldots \ldots \ldots \ldots \ldots \ldots \\
\text { 65. Wake Forest (N.C.) } \ldots \ldots \ldots \ldots \ldots \ldots\end{array}$ & $\begin{array}{r}96,608 \\
27,250 \\
114,893 \\
113,639 \\
\mathbf{4 5}, 714\end{array}$ & $\begin{array}{l}21,166 \\
11,900 \\
39,307 \\
46,171 \\
28,540\end{array}$ & $\begin{array}{r}128,648 \\
39,750 \\
162,475 \\
165,632 \\
76,143\end{array}$ & $\begin{array}{l}7,100 \\
6,000 \\
6,300 \\
\ldots \ldots \\
6,500\end{array}$ & $\begin{array}{l}4,800 \\
\ldots \ldots \\
\cdots \cdots \\
\cdots \cdots \\
\cdots \cdots\end{array}$ & $\begin{array}{l}3,200 \\
5,500 \\
3,936 \\
3,200\end{array}$ & $\begin{array}{l}4,000 \\
3,300 \\
5,800 \\
4,920 \\
3,200\end{array}$ & $\begin{array}{l}6 \\
1 \\
4 \\
4 \\
2\end{array}$ \\
\hline $\begin{array}{l}\text { 66. Wellesley (Mass.) } \ldots \ldots \ldots \ldots \ldots \ldots \ldots \\
\text { 67. Wheaton (IIl.) } \ldots \ldots \ldots \ldots \ldots \ldots \ldots \\
\text { 68. Wichita (Kan.) } \ldots \ldots \ldots \ldots \ldots \ldots \\
\text { 69. William \& Mary (Va.) } \ldots \ldots \ldots \ldots \ldots \ldots \\
\text { 70. Williams (Mass.) } \ldots \ldots \ldots \ldots \ldots \ldots \ldots\end{array}$ & $\begin{array}{l}99,695 \\
20,050 \\
49,200 \\
68,685 \\
40,380\end{array}$ & $\begin{array}{l}30,000^{4} \\
10,000 \\
29,700 \\
24,900 \\
25,500\end{array}$ & $\begin{array}{r}139,659 \\
32,677 \\
81,205 \\
99,422 \\
68,880\end{array}$ & $\begin{array}{l}6,500 \\
4,250 \\
5,800 \\
6,432 \\
7,250\end{array}$ & $\begin{array}{l}5,000 \\
3,960 \\
\cdots \cdots \\
3,100\end{array}$ & $\begin{array}{l}2,700 \\
3,400 \\
3,936 \\
4,200\end{array}$ & $\begin{array}{l}3,190 \\
4,200 \\
4,704 \\
4,500\end{array}$ & $\begin{array}{l}3 \\
4 \\
4 \\
2\end{array}$ \\
\hline 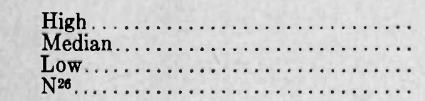 & $\begin{array}{r}199,800 \\
45,126 \\
17,178 \\
68\end{array}$ & $\begin{array}{r}93,315 \\
19,900 \\
5,000 \\
67\end{array}$ & $\begin{array}{r}299,300 \\
69,825 \\
28,917 \\
69\end{array}$ & $\begin{array}{r}8,040 \\
6,000 \\
4,000 \\
52\end{array}$ & $\begin{array}{r}7,500 \\
4,650 \\
3,000 \\
24\end{array}$ & $\begin{array}{r}5,500 \\
3,600 \\
2,400 \\
46\end{array}$ & $\begin{array}{r}6,500 \\
4,380 \\
3,190 \\
39\end{array}$ & $\begin{array}{r}13 \\
3 \\
1 \\
45\end{array}$ \\
\hline
\end{tabular}

1 Librarian on 9-months leave. 2 Plus pay for summer teaching. 3 Binding included in salaries and wages. 4 Does not include income from endowment. $5 \$ 5,000-\$ 6,500(2)$. 6 Includes other operating expenses. 7 Plus 39 part-time. 89 months. $9 \$ 3,660-\$ 6,000(6)$. 10 Plus one librarian not paid from library budget. $11 \$ 2,700-\$ 3,450(3), 1210$ months. $13 \$ 3,500-\$ 5,500(1) .1410$ months. $15 \$ 3,000-\$ 5,580$. 16 $\$ 4,163-\$ 5,478(3)$. 17 $\$ 3,300-$ $\$ 3,600$ (3). $18 \$ 3,800-\$ 4,402(6)$. $19 \$ 3,720-\$ 5,320$ (2). $20 \$ 3,200-\$ 3,600(2)$. $21 \$ 4,200-\$ 5,000(2)$. $2238.5-40$ hours weekly. 2337.5 non-professional 


\section{and Salary Statistics-I954-55 (Group II)}

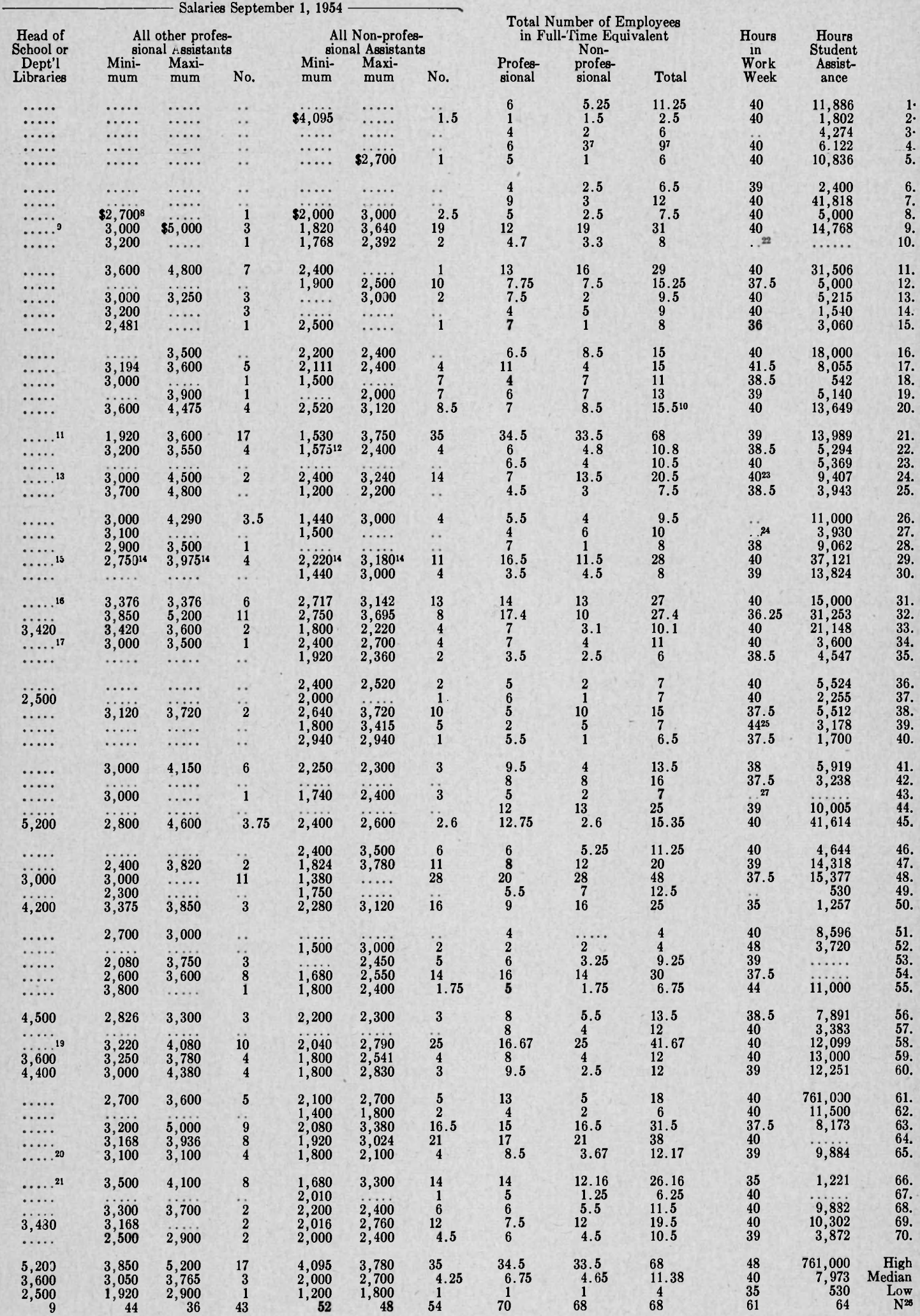

2435 -38 hours weekly. 2540 non-professional. 26 Number of libraries on which high, median and low a-e based. $2735-40$ hours weekly. $28 \$ 3,600-$ $\$ 4,800$ (1). $29 \$ 4,000-\$ 5,500$ (2). $30 \$ 3,960-\$ 4,860$ (2). $31 \$ 3,635-\$ 3,980$ (2). $32 \$ 4,250-\$ 4,500$ (2). $33 \$ 3,300-\$ 3,900(2)$. $34 \$ 6,144-\$ 7,680$. $35 \$ 4,920-$ $\$ 6,148(1)$. 


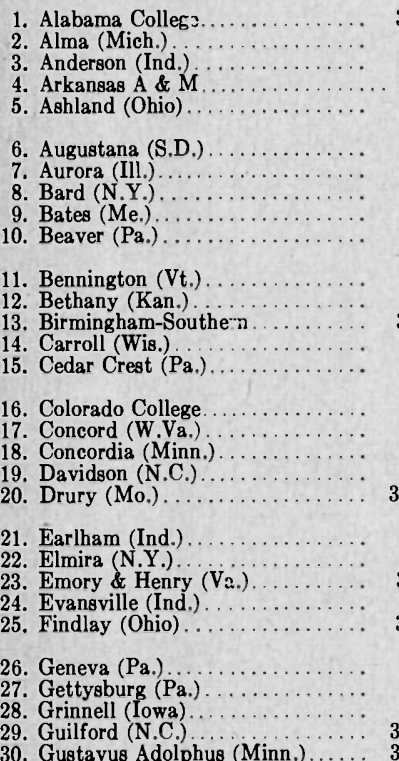

Fiscal
Year
Ending

31Ag54
30Je54
30Je54
30Je54
1Je54

30Je54
30Je54
30Je54
30Je54
30Je54

30Je54
31Jy54
31Ag54
30Je54
1Je54

Student Enrollment

Total Total

graduates ates

$\begin{array}{ll}562 & \ldots \\ 502 & \\ 902 & 55 \\ 672 & \ldots \\ 325 & \text { i5 } \\ 649 & \ldots\end{array}$

$30 \mathrm{Je} 54$

$30 \mathrm{Je} 54$

$31 \mathrm{My} 54$

$30 \mathrm{Je} 54$

31 Ag5 4

$30 \mathrm{Je} 54$
$30 \mathrm{Ap5} 4$

$30 \mathrm{Je} 54$

30Je54

$1 \mathrm{My} 54$

31 My54
31 My54

31. Hillsdale (Mich.)

32. Hiram (Ohio)

33. Hollins ( $\mathrm{Va}$.)

35. Hood (Md.) H.Y.

$30 \mathrm{Je} 54$

$30 \mathrm{Je} 54$

$30 \mathrm{Je} 54$

15Jy54

30 A 54

36. Huntington (Ind.)

37. Illinois College.

38. Illinois Wesleyan.

39. Immaculate Hea t

$31 \mathrm{Jy} 54$

30Je54

$31 \mathrm{Jy} 54$

30Je54

41. Juniata ( $\mathrm{Pa}$.)

43. Lebanon Valley (Pa)

44. Linfield (Ore.)

15Je54

$30 \mathrm{Je} 54$

$30 \mathrm{Ap} 54$

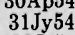

$31 \mathrm{Jy} 54$
$30 \mathrm{Je} 54$

46. Manchester (Ind.)

47. Millikin (IIl.)

48. Millsaps (Miss.)

49. Morningside (Iowa)

$31 \mathrm{Jy} 54$

$30 \mathrm{Je} 54$

$30 \mathrm{Je} 54$

$31 \mathrm{Jy} 54$
$30 \mathrm{Je} 54$

649

486
203
846

846
488

327
285
714
544

7

52

$\begin{array}{rlr}907 & 23 & 157,888 \\ 931 & \ldots & 23,375 \\ 1,107 & \ldots & 43,255 \\ 824 & \ldots & 62,736 \\ 681 & & 35,877\end{array}$

$3 \quad 82,398$

623
252
514

1,052

138

618

1,206
832

491
897

505

505
454

473

500
571

171

254
720
450
296

12

11
103

82,398

61,379
31,870

31,870
36,702

$\begin{array}{rr}36,702 & 2,509^{\mathrm{g}} \\ 17,929 & 840^{\mathrm{d}}\end{array}$

54,045

72,799
117,275

36,988

,

34,434

72,686
52,828

40,237

34,697

18,241

42,458

53,278
57,899

23,190

591

755

613
450
835

74,625

55,815

$\begin{array}{ll}30 & 45,929 \\ \cdots & 99,412\end{array}$

99,412

$\begin{array}{lll}660 & & 43,761 \\ 879 & 6 & 52,577 \\ 704 & & 36,839\end{array}$

36,839
64,700

90,466

$31 \mathrm{My} 54$

51. Nebraska Wesleyan

52. New Mexico Highla!d:

53. Parsons (Iowa)

54. Randolph-Macon (Va.)

$30 \mathrm{Je} 54$

$31 \mathrm{Ag} 54$

$30 \mathrm{Ap} 54$

56. Rosary (Ill.).

57. Seneca, Coll. of the (N.Y.)

58. Seton Hill ( $\mathrm{Pa}$.)

59. Springfield (Mass.)

$30 \mathrm{Je} 54$
$30 \mathrm{Je5} 4$

31 Ag5 4

$31 \mathrm{Ag} 54$
$30 \mathrm{Je5} 4$

61. Talladega (Ga.).

62. Trinity (D.C.)

63. Union (Neb.)

. $30 \mathrm{Je54}$

64. Ursinus $(\mathrm{Pa}.) \ldots \ldots \ldots \ldots \ldots \ldots$ 30Je54

65. Washington \& Jefferson (Pa.) ... 30Je54

66. Washington \& Lee (Va.)

67. Wells (N.Y.)

68. Wilberforce (Ohio)

70. Willamette (Ore.)

30Je54

30Je54

$30 \mathrm{Je} 54$
$31.1 \mathrm{~g} 54$

Migh.

Modian.......

N5

40,562

$\begin{array}{lll}510 & 43 & 57,099 \\ 240 & \ldots & 29,204\end{array}$

596

78,024

54,888

$\begin{array}{lll}608 & 86 & 72,000\end{array}$

$\begin{array}{lll}869 & \cdots & 93,415 \\ 455 & \cdots & 36,115\end{array}$

113

44,965
82,131

Volumes

News-

Periodi-

papers cals

Cur- Cur-
rently rently
Re-

Added ceived ceived

Staff

Student

Est. non-

$1,520^{\mathrm{a}}$
$1,075^{\mathrm{b}}$
$1,822^{\mathrm{a}}$
$2,347^{\mathrm{a}}$
$800^{\mathrm{e}}$

$18 \quad 428$

\begin{tabular}{rr}
6 & $196^{\mathrm{j}}$ \\
5 & $246^{\mathrm{j}}$ \\
\hline
\end{tabular}

$15,300 \quad 2,435$

$\begin{array}{rr}5,150 & 591 \\ 8,761 & 2,736\end{array}$

$\begin{array}{rr}7,750 & 1,800 \\ 8,700 & 580\end{array}$

$1,191^{\mathrm{e}}$

$622^{\mathrm{b}}$
$2,347^{\mathrm{k}}$

$2,663^{\mathrm{k}}$

7,000

6,861
15,960

15,960

1,810

2,487

1,500
1,899
540

$1,092^{\mathrm{a}}$

$1,500^{\mathrm{b}}$

$1,435^{\mathrm{c}}$
$878^{\mathrm{k}}$

15,371

3,850

11,565

6,055
4,983

601

1,473

4,186
2,173

19,736

$855^{\circ}$

$1,643^{\mathrm{g}}$

$1,900^{\mathrm{b}}$

16,080

9,425

13,890
9,596

$\begin{array}{llll}2,455^{\mathrm{a}} & 5 & 362^{\mathrm{J}} & 11,957\end{array}$

11,957
3,220

$\begin{array}{rrr}909^{\mathrm{a}} & 6 & 242^{\mathrm{j}} \\ 1,378^{\mathrm{e}} & 14 & 338^{\mathrm{j}}\end{array}$

$1,466^{\mathrm{k}}$

$2,537^{\mathrm{b}}$

$1,618^{\mathrm{d}}$

$1,754^{\mathrm{k}}$
$2,715^{\mathrm{a}}$

2,871

1,918
2,206

3,200

2,02

2,024
1,478
2,750

2,759
1,447

9,800

3,520

11,045

11,325

21,734
8,714

11,756

1,056

4,074

1,328
608

2,096

$974^{\mathrm{a}}$

$2,150^{\mathrm{h}}$

1,660

$2,576^{\mathrm{a}}$
$1,370^{\mathrm{B}}$

5,800

10,675

10,900

300
$\mathbf{5 8}$

3,276

2,210

$1,194^{\mathrm{e}}$

$901^{\mathrm{b}}$
$1,871^{\mathrm{g}}$
$3,551^{\mathrm{k}}$

$3,551^{\mathrm{k}}$
$1,218^{\mathrm{B}}$

3,454

6,200

10,815

6,500
5,352

1,050

3,744

1,752

14,550

$1,807^{\mathrm{b}}$

$1,482^{\mathrm{e}}$

$2,491^{\mathrm{b}}$

$2,424^{\mathrm{e}}$

6,158
12,977

2,128

1,070

2,980

$1,202^{\mathrm{b}}$

$1,214^{\mathrm{d}}$

$1,485^{\mathrm{d}}$

$2,887^{\mathrm{a}}$

3,234

7,150

7,633

11,550

5,800
$12,600^{2}$

3,588

2,161

2,046

$562^{\mathrm{k}}$

$2,449^{\mathrm{e}}$

$1,543^{\mathrm{a}}$

6,200

880
5,180

5,180

1,222
255

1,807

$1,937^{\mathrm{b}}$

2,727

$1,013^{\mathrm{b}}$

$1,365^{\mathrm{a}}$
$1,912^{\mathrm{a}}$

1,616

2,407

1,150
1,463

463
791

12,034

19,862

1,128

10,480
2,100

2,441

6,005

2,062
1,778

9,600

64,615

$4,015^{\mathrm{b}}$

$1,771^{\mathrm{e}}$

$801^{\mathrm{a}}$
$1,936^{\mathrm{g}}$

9,570

800

$\begin{array}{ll}157,080 & 3,445^{\mathrm{h}} \\ 115,869 & 1,312^{\mathrm{k}}\end{array}$

$\begin{array}{rl}24,967 & 1,967 \mathrm{e} \\ 54,527 & \mathrm{l}, 621^{\mathrm{B}}\end{array}$

$\begin{array}{ll}54,527 & 1,621^{\mathrm{B}} \\ 29,764 & 1,050^{\circ}\end{array}$

19,356

15,300
6,600

6,600
12,266

674
3,516

3,117
1,510

$\begin{array}{rr}21,734 & 6,005 \\ 9,596 & 1,778 \\ 3,454 & 255 \\ 66 & 69\end{array}$

2,000

1,067
510 


\begin{tabular}{|c|c|c|c|c|c|c|c|c|c|c|c|c|c|}
\hline $\begin{array}{l}\text { Salaries } \\
\text { Est. } \\
\text { non-8al. } \\
\text { and } \\
\text { Student } \\
\text { Service }\end{array}$ & $\begin{array}{c}\text { Books } \\
\text { and } \\
\text { Other } \\
\text { Materials }\end{array}$ & Binding & $\begin{array}{l}\text { Total } \\
\text { Books } \\
\text { and } \\
\text { Binding }\end{array}$ & $\begin{array}{l}\text { Other } \\
\text { Expendi- } \\
\text { tures }\end{array}$ & $\begin{array}{c}\text { Total } \\
\text { Operating } \\
\text { Expenses }\end{array}$ & $\begin{array}{c}\text { Per } \\
\text { Student } \\
\text { Operating } \\
\text { Expendi- } \\
\text { tures }\end{array}$ & $\begin{array}{c}\text { Total } \\
\text { College } \\
\text { Expenditures }\end{array}$ & $\begin{array}{c}\text { Ratio } \\
\text { Library Ex- } \\
\text { penditures to } \\
\text { Total College } \\
\text { Expenditures } \\
\text { (Per Cent) }\end{array}$ & $\begin{array}{l}\text { Salaries } \\
\text { and } \\
\text { Wages }\end{array}$ & $\begin{array}{c}\text { Books, } \\
\text { Periodi- } \\
\text { cals \& } \\
\text { Binding }\end{array}$ & $\begin{array}{l}\text { Other } \\
\text { Operat- } \\
\text { ing } \\
\text { Ex } \\
\text { penses }\end{array}$ & $\begin{array}{c}\text { Total } \\
\text { Library } \\
\text { Operating } \\
\text { Budget }\end{array}$ & \\
\hline $\begin{array}{r}\$ 17,735 \\
5,741 \\
11,497 \\
9,550 \\
9,280\end{array}$ & $\begin{array}{r}\mathbf{6}, 993 \\
4,980 \\
5,545 \\
10,000 \\
3,406\end{array}$ & $\begin{array}{r}584 \\
290 \\
319 \\
500 \\
473\end{array}$ & $\begin{array}{r}7,577 \\
5,270 \\
5,864 \\
10,500 \\
3,879\end{array}$ & $\begin{array}{r}\$ 1,116 \\
209 \\
252 \\
1,500 \\
244\end{array}$ & $\begin{array}{r}\$ 26,429 \\
11,220 \\
17,613 \\
21,550 \\
13,403\end{array}$ & $\begin{array}{r}\$ 7.03 \\
22.35 \\
18.40 \\
32.07 \\
39.42\end{array}$ & $\begin{array}{r}\$ 815,300 \\
443,584 \\
412,845 \\
450,000 \\
341,381\end{array}$ & $\begin{array}{r}\$ 3.24 \\
2.53 \\
4.27 \\
4.79 \\
3.93\end{array}$ & $\begin{array}{r}\$ 17,750 \\
5,910 \\
12,500 \\
10,800 \\
9,400\end{array}$ & $\begin{array}{r}7,550 \\
5,450 \\
6,250 \\
5,200 \\
4,175\end{array}$ & $\begin{array}{r}\$ 1,000 \\
500 \\
1,135 \\
1,500 \\
750\end{array}$ & $\begin{array}{r}\$ 26,300 \\
11,860 \\
19,885 \\
17,500 \\
14,325\end{array}$ & $\begin{array}{l}1 . \\
2 . \\
3 . \\
4 . \\
5 .\end{array}$ \\
\hline $\begin{array}{r}8,810 \\
9,348 \\
17,460 \\
18,482 \\
8,923\end{array}$ & $\begin{array}{l}5,609 \\
2,485 \\
8,215 \\
9,386 \\
2,544\end{array}$ & $\begin{array}{r}346 \\
\because 2,040 \\
1,400 \\
456\end{array}$ & $\begin{array}{r}5,955 \\
2,485 \\
10,255 \\
10,786 \\
3,000\end{array}$ & $\begin{array}{r}200 \\
142 \\
3,755 \\
560 \\
200\end{array}$ & $\begin{array}{l}14,965 \\
11,975 \\
31,470 \\
29,828 \\
12,123\end{array}$ & $\begin{array}{r}23.06 \\
24.64 \\
155.02 \\
35.26 \\
24.84\end{array}$ & $\begin{array}{l}353,003 \\
207,435 \\
623,000 \\
738,181\end{array}$ & $\begin{array}{l}4.24 \\
5.77 \\
5.05 \\
1.64 \\
1.64\end{array}$ & $\begin{array}{c}10,000 \\
10,295 \\
14,450 \\
17,750^{4} \\
9,323\end{array}$ & $\begin{array}{l}6,000 \\
2,750 \\
8,450 \\
8,498^{4} \\
5,000\end{array}$ & $\begin{array}{r}300 \\
3,230 \\
800^{4} \\
1,000\end{array}$ & $\begin{array}{l}16,000 \\
13,345 \\
27,630 \\
27,048^{4} \\
15,323\end{array}$ & $\begin{array}{r}6 . \\
7 . \\
8 . \\
9 . \\
10 .\end{array}$ \\
\hline $\begin{array}{r}15,972 \\
5,323 \\
15,751 \\
8,228 \\
5,412\end{array}$ & $\begin{array}{l}3,865 \\
2,500 \\
6,336 \\
5,990 \\
\ldots \ldots .\end{array}$ & $\begin{array}{r}860 \\
40 \\
1,157 \\
403 \\
\ldots \ldots\end{array}$ & $\begin{array}{l}4,725 \\
2,540 \\
7,493 \\
6,393 \\
3,245\end{array}$ & $\begin{array}{r}285 \\
196 \\
1,319 \\
656 \\
197\end{array}$ & $\begin{array}{r}20,982 \\
8,059 \\
24,563 \\
15,277 \\
8,854\end{array}$ & $\begin{array}{l}62.82 \\
28.28 \\
32.07 \\
28.08 \\
22.02\end{array}$ & $\begin{array}{l}482,418 \\
223,578 \\
531,271 \\
386,369 \\
331,540\end{array}$ & $\begin{array}{l}4.35 \\
3.60 \\
4.62 \\
3.95 \\
2.67\end{array}$ & $\begin{array}{r}16,450 \\
6,500 \\
15,505 \\
8,625 \\
4,983\end{array}$ & $\begin{array}{r}4,850 \\
3,130 \\
7,150 \\
6,500 \\
3,927\end{array}$ & $\begin{array}{r}350 \\
370 \\
1,250 \\
700 \\
273\end{array}$ & $\begin{array}{r}21,650 \\
10,000 \\
24,455 \\
15,825 \\
8,283\end{array}$ & $\begin{array}{l}11 . \\
12 . \\
13 . \\
14 . \\
15 .\end{array}$ \\
\hline $\begin{array}{l}22,607 \\
17,998 \\
11,631 \\
17,090 \\
11,066\end{array}$ & $\begin{array}{l}7,622 \\
4,622 \\
4,655 \\
9,186 \\
6,822\end{array}$ & $\begin{array}{r}1,630 \\
944 \\
465 \\
1,380 \\
548\end{array}$ & $\begin{array}{r}9,252 \\
5,566 \\
5,120 \\
10,566 \\
7,370\end{array}$ & $\begin{array}{r}814 \\
765 \\
1,296 \\
2,552 \\
781\end{array}$ & $\begin{array}{l}32,673 \\
24,329 \\
18,047 \\
30,208 \\
19,217\end{array}$ & $\begin{array}{l}35.13 \\
26.13 \\
16.30 \\
36.66 \\
28.22\end{array}$ & $\begin{array}{l}677,011 \\
468,819 \\
627,296 \\
706,698 \\
355,325\end{array}$ & $\begin{array}{l}4.83 \\
5.19 \\
2.88 \\
4.27 \\
5.41\end{array}$ & $\begin{array}{l}16,900 \\
24,396 \\
12,750 \\
18,250 \\
12,375\end{array}$ & $\begin{array}{r}9,500 \\
6,400 \\
5,575 \\
10,400 \\
7,300\end{array}$ & $\begin{array}{r}5,600 \\
100 \\
1,755 \\
3,250 \\
800\end{array}$ & $\begin{array}{r}32,000 \\
24,396 \\
20,080 \\
32,175 \\
\cdot 20,475\end{array}$ & $\begin{array}{l}16 . \\
17 . \\
18 . \\
19 . \\
20 .\end{array}$ \\
\hline $\begin{array}{r}13,981 \\
8,698 \\
6,659 \\
11,247 \\
4,047\end{array}$ & $\begin{array}{l}5,757 \\
3,324 \\
6,436 \\
5,709 \\
2,672\end{array}$ & $\begin{array}{r}721 \\
231 \\
\cdots \\
732 \\
122\end{array}$ & $\begin{array}{l}6,478 \\
3,555 \\
6,436 \\
6,441 \\
2,794\end{array}$ & $\begin{array}{r}952 \\
384 \\
1,859 \\
125\end{array}$ & $\begin{array}{r}21,411 \\
12,637 \\
13,095 \\
19,547 \\
6,966\end{array}$ & $\begin{array}{l}34.20 \\
50.15 \\
25.48 \\
16.43 \\
26.39\end{array}$ & $\begin{array}{l}\mathbf{5 7 0}, 128 \\
356,645 \\
336,784 \\
653,800 \\
219,885\end{array}$ & $\begin{array}{l}3.76 \\
3.54 \\
3.89 \\
2.99 \\
3.17\end{array}$ & $\begin{array}{r}10,400 \\
9,145 \\
9,000 \\
11,200 \\
3,520\end{array}$ & $\begin{array}{l}6,500 \\
4,000 \\
7,000 \\
5,700 \\
2,300\end{array}$ & $\begin{array}{r}1,250 \\
500 \\
\cdots \ldots 00 \\
600 \\
200\end{array}$ & $\begin{array}{r}18,150 \\
13,645 \\
16,000 \\
17,500 \\
6,020\end{array}$ & $\begin{array}{l}21 . \\
22 . \\
23 . \\
24 . \\
25 .\end{array}$ \\
\hline $\begin{array}{r}12,101 \\
15,399 \\
23,062 \\
9,322 \\
14,752\end{array}$ & $\begin{array}{r}3,400 \\
14,070 \\
7,102 \\
4,434 \\
8,036\end{array}$ & $\begin{array}{r}216 \\
745 \\
1,229 \\
366 \\
377\end{array}$ & $\begin{array}{r}3,616 \\
14,815 \\
8,331 \\
4,800 \\
8,413\end{array}$ & $\begin{array}{r}428 \\
1,022 \\
1,664 \\
730\end{array}$ & $\begin{array}{l}16,145 \\
30,214 \\
32,415 \\
15,786 \\
23,895\end{array}$ & $\begin{array}{l}26.12 \\
25.05 \\
38.96 \\
32.15 \\
26.64\end{array}$ & $\begin{array}{r}636,066 \\
864,685 \\
1,235,526 \\
229,320 \\
595,300\end{array}$ & $\begin{array}{l}2.54 \\
3.49 \\
2.62 \\
6.88 \\
4.01\end{array}$ & $\begin{array}{r}10,107 \\
15,625 \\
23,475 \\
9,321 \\
17,930\end{array}$ & $\begin{array}{l}6,073 \\
8,600 \\
8,500 \\
4,799 \\
8,740\end{array}$ & $\begin{array}{r}350 \\
4,000 \\
830 \\
4,179 \\
900\end{array}$ & $\begin{array}{l}16,530 \\
28,225 \\
32,805 \\
18,299 \\
27,570\end{array}$ & $\begin{array}{l}26 . \\
27 . \\
28 . \\
29 . \\
30 .\end{array}$ \\
\hline $\begin{array}{r}6,100 \\
12,135 \\
12,252 \\
14,176 \\
12,165\end{array}$ & $\begin{array}{l}3,370 \\
6,710 \\
5,906 \\
8,596 \\
3,906\end{array}$ & $\begin{array}{r}438 \\
782 \\
592 \\
825 \\
1,059\end{array}$ & $\begin{array}{l}3,808 \\
7,492 \\
6,498 \\
9,421 \\
4,965\end{array}$ & $\begin{array}{r}120 \\
465 \\
456 \\
983 \\
1,210\end{array}$ & $\begin{array}{l}10,028 \\
20,092 \\
19,206 \\
24,580 \\
18,340\end{array}$ & $\begin{array}{l}19.86 \\
44.26 \\
40.60 \\
49.16 \\
32.12\end{array}$ & $\begin{array}{l}361,667 \\
455,493 \\
512,497 \\
645,495 \\
275,635\end{array}$ & $\begin{array}{l}2.77 \\
4.41 \\
3.75 \\
3.81 \\
6.65\end{array}$ & $\begin{array}{r}5,600 \\
14,200 \\
11,965 \\
14,400 \\
13,000\end{array}$ & $\begin{array}{l}4,000 \\
9,000 \\
6,022 \\
6,000 \\
5,200\end{array}$ & $\begin{array}{r}100 \\
1,000 \\
270 \\
1,300 \\
1,597\end{array}$ & $\begin{array}{r}9,700 \\
24,200 \\
18,257 \\
21,700 \\
19,857\end{array}$ & $\begin{array}{l}31 . \\
32 . \\
33 . \\
34 . \\
35 .\end{array}$ \\
\hline $\begin{array}{r}4,696 \\
7,250 \\
14,559 \\
21,050 \\
7,104\end{array}$ & $\begin{array}{l}3,955 \\
2,974 \\
5,374 \\
6,888 \\
4,044\end{array}$ & $\begin{array}{l}153 \\
396 \\
770 \\
700 \\
438\end{array}$ & $\begin{array}{l}4,108 \\
3,370 \\
6,144 \\
7,588 \\
4,482\end{array}$ & $\begin{array}{r}240 \\
379 \\
1,597 \\
661 \\
1,828\end{array}$ & $\begin{array}{r}9,044 \\
10,999 \\
22,300 \\
29,299 \\
12,414\end{array}$ & $\begin{array}{l}49.42 \\
43.30 \\
30.51 \\
52.98 \\
41.94\end{array}$ & \begin{tabular}{l}
157,231 \\
247,286 \\
623,688 \\
\hdashline$\ldots \dddot{264}, 981$
\end{tabular} & \begin{tabular}{l}
5.75 \\
4.45 \\
3.58 \\
\hdashline .68
\end{tabular} & $\begin{array}{r}4,600 \\
7,340 \\
17,100 \\
24,850 \\
5,100\end{array}$ & $\begin{array}{l}5,500 \\
3,400 \\
5,430 \\
7,500 \\
4,500\end{array}$ & $\begin{array}{r}104 \\
325 \\
1,000 \\
1,000 \\
1,900\end{array}$ & $\begin{array}{l}10,204 \\
11,065 \\
23,500 \\
33,350 \\
11,500\end{array}$ & $\begin{array}{l}36 . \\
37 . \\
38 . \\
39 . \\
40 .\end{array}$ \\
\hline $\begin{array}{r}8,286 \\
13,944 \\
13,396 \\
8,180 \\
19,248\end{array}$ & $\begin{array}{l}5,211 \\
5,534 \\
5,645 \\
2,951 \\
5,491\end{array}$ & $\begin{array}{r}493 \\
577 \\
1,053 \\
198 \\
632\end{array}$ & $\begin{array}{l}5,704 \\
6,111 \\
6,698 \\
3,149 \\
6,123\end{array}$ & $\begin{array}{r}373 \\
1,082 \\
644 \\
\ldots \ldots \\
535\end{array}$ & $\begin{array}{l}14,363 \\
21,137 \\
20,738 \\
11,329 \\
25,906\end{array}$ & $\begin{array}{l}24.30 \\
28.00 \\
33.83 \\
23.60 \\
31.03\end{array}$ & $\begin{array}{l}426,990 \\
647,575 \\
373,752 \\
\ldots 7 . \ldots \\
476,452\end{array}$ & \begin{tabular}{l}
3.36 \\
3.26 \\
5.55 \\
\hdashline .44 \\
5.44
\end{tabular} & $\begin{array}{r}9,500 \\
15,550 \\
14,042 \\
8,380 \\
20,400\end{array}$ & $\begin{array}{l}6,070 \\
7,700 \\
6,222 \\
3,500 \\
6,600\end{array}$ & $\begin{array}{r}1,200 \\
550 \\
\cdots \\
500\end{array}$ & $\begin{array}{l}15,570 \\
24,450 \\
21,314 \\
11,880 \\
27,500\end{array}$ & $\begin{array}{l}41 . \\
42 . \\
43 . \\
44 . \\
45 .\end{array}$ \\
\hline $\begin{array}{r}10,384 \\
11,221 \\
12,350 \\
7,961 \\
14,646\end{array}$ & $\begin{array}{l}4,538 \\
4,254 \\
3,806 \\
3,240 \\
6,829\end{array}$ & $\begin{array}{l}516 \\
570 \\
789 \\
247 \\
950\end{array}$ & $\begin{array}{l}\mathbf{5}, 054 \\
4,824 \\
4,595 \\
3,487 \\
7,779\end{array}$ & $\begin{array}{r}323 \\
2,186 \\
2,927 \\
132 \\
737\end{array}$ & $\begin{array}{l}15,761 \\
18,231 \\
19,872 \\
11,580 \\
23,162\end{array}$ & $\begin{array}{l}23.88 \\
20.60 \\
28.22 \\
19.79 \\
37.85\end{array}$ & \begin{tabular}{l}
306,148 \\
579,018 \\
461,126 \\
\hdashline 555,596
\end{tabular} & \begin{tabular}{l}
5.15 \\
3.15 \\
4.31 \\
\hdashline .17
\end{tabular} & $\begin{array}{r}10,000 \\
10,318 \\
12,955 \\
8,000 \\
14,960\end{array}$ & $\begin{array}{l}5,425 \\
5,450 \\
4,375 \\
4,200 \\
8,600\end{array}$ & $\begin{array}{r}925 \\
2,250 \\
600 \\
300 \\
850\end{array}$ & $\begin{array}{l}16,350 \\
18,018 \\
17,930 \\
12,500 \\
24,410\end{array}$ & $\begin{array}{l}46 . \\
47 . \\
48 . \\
49 . \\
50 .\end{array}$ \\
\hline $\begin{array}{r}7,000 \\
23,250 \\
6,685 \\
19,055 \\
7,370\end{array}$ & $\begin{array}{l}2,400 \\
5,500 \\
3,151 \\
5,548 \\
4,298\end{array}$ & $\begin{array}{r}200 \\
1,433 \\
392 \\
1,110 \\
612\end{array}$ & $\begin{array}{l}2,600 \\
6,933 \\
3,543 \\
6,658 \\
4,910\end{array}$ & $\begin{array}{r}150 \\
\cdots 164 \\
810 \\
521\end{array}$ & $\begin{array}{r}9,750 \\
30,183 \\
10,392 \\
26,523 \\
12,801\end{array}$ & $\begin{array}{l}54.58 \\
43.30 \\
44.50 \\
26.18\end{array}$ & $\begin{array}{l}320,213 \\
662,072 \\
221,536 \\
654,511 \\
430,146\end{array}$ & $\begin{array}{l}3.04 \\
4.56 \\
4.69 \\
4.05 \\
2.98\end{array}$ & $\begin{array}{r}9,020 \\
22,400 \\
7,200 \\
19,010 \\
9,400\end{array}$ & $\begin{array}{r}2,900 \\
12,780 \\
3,250 \\
6,700 \\
6,300\end{array}$ & $\begin{array}{r}100 \\
\cdots 100 \\
\cdots \\
600\end{array}$ & $\begin{array}{l}12,020 \\
31,200 \\
10,550 \\
25,710 \\
16,300\end{array}$ & $\begin{array}{l}51 . \\
52 . \\
53 . \\
54 . \\
55 .\end{array}$ \\
\hline $\begin{array}{r}13,516 \\
20,237 \\
9,150 \\
13,497 \\
20,653\end{array}$ & $\begin{array}{l}7,355 \\
8,712 \\
3,208 \\
5,000 \\
7,972\end{array}$ & $\begin{array}{r}1,087 \\
1,569 \\
336 \\
600 \\
1,212\end{array}$ & $\begin{array}{r}8,443 \\
10,281 \\
3,544 \\
5,600 \\
9,184\end{array}$ & $\begin{array}{r}531 \\
1,516 \\
722 \\
536 \\
751\end{array}$ & $\begin{array}{l}22,490 \\
32,034 \\
13,416 \\
19,633 \\
30,588\end{array}$ & $\begin{array}{l}32.41 \\
36.86 \\
29.49 \\
21.13 \\
67.67\end{array}$ & $\begin{array}{l}453,547 \\
678,452 \\
293,975 \\
960,000 \\
781,523\end{array}$ & $\begin{array}{l}4.96 \\
4.72 \\
4.56 \\
2.05 \\
3.91\end{array}$ & $\begin{array}{r}17,500 \\
21,218 \\
9,100 \\
15,000 \\
20,860\end{array}$ & $\begin{array}{r}8,500 \\
9,500 \\
4,300 \\
5,500 \\
10,300\end{array}$ & $\begin{array}{r}750 \\
2,000 \\
100 \\
500 \\
1,741\end{array}$ & $\begin{array}{l}26,750 \\
32,718 \\
13,500 \\
21,000 \\
32,900\end{array}$ & $\begin{array}{l}56 . \\
57 . \\
58 . \\
59 . \\
60 .\end{array}$ \\
\hline $\begin{array}{l}11,608 \\
14,141 \\
10,589 \\
11,632 \\
12,718\end{array}$ & $\begin{array}{l}3,931 \\
7,579 \\
5,493 \\
3,639 \\
4,224\end{array}$ & $\begin{array}{r}300 \\
413 \\
1,512 \\
355 \\
265\end{array}$ & $\begin{array}{l}4,231 \\
7,992 \\
7,005 \\
3,994 \\
4,489\end{array}$ & $\begin{array}{r}332 \\
1,078 \\
2,105 \\
255 \\
2,600\end{array}$ & $\begin{array}{l}16,171 \\
23,211 \\
19,699 \\
15,881 \\
19,807\end{array}$ & $\begin{array}{l}55.00 \\
48.46 \\
27.98 \\
23.39 \\
42.14\end{array}$ & $\begin{array}{l}432,348 \\
373,934 \\
448,722 \\
512,000\end{array}$ & $\begin{array}{l}3.74 \\
6.21 \\
4.39 \\
3.87 \\
3.87\end{array}$ & $\begin{array}{l}11,833 \\
14,500 \\
11,079 \\
11,804 \\
15,000\end{array}$ & $\begin{array}{l}4,231 \\
9,800 \\
5,570 \\
4,750 \\
5,250\end{array}$ & $\begin{array}{r}336 \\
1,200 \\
1,850 \\
200 \\
5,650\end{array}$ & $\begin{array}{l}16,400 \\
25,500 \\
18,490 \\
16,755 \\
20,900\end{array}$ & $\begin{array}{l}61 . \\
62 . \\
63 . \\
64 . \\
65 .\end{array}$ \\
\hline $\begin{array}{r}20,156 \\
15,974 \\
10,116 \\
15,383 \\
5,779\end{array}$ & $\begin{array}{r}10,233 \\
8,475 \\
4,000 \\
6,000 \\
3,333\end{array}$ & $\begin{array}{r}1,461 \\
1,125 \\
400 \\
2,277 \\
263\end{array}$ & $\begin{array}{r}11,694 \\
9,600 \\
4,400 \\
8,277 \\
3,596\end{array}$ & $\begin{array}{r}955 \\
1,788 \\
\cdots \quad 7 \\
527 \\
726\end{array}$ & $\begin{array}{l}32,805 \\
27,362 \\
14,516 \\
24,187 \\
16,101\end{array}$ & $\begin{array}{l}36.09 \\
84.19 \\
52.59 \\
22.25 \\
31.57\end{array}$ & $\begin{array}{l}957,601 \\
534,981 \\
200,000 \\
559,815 \\
299,477\end{array}$ & $\begin{array}{l}3.43 \\
5.11 \\
7.26 \\
4.32 \\
5.38\end{array}$ & $\begin{array}{r}21,800 \\
15,879 \\
10,000 \\
15,555 \\
6,600\end{array}$ & $\begin{array}{r}11,500 \\
10,500 \\
5,000 \\
6,750 \\
3,300\end{array}$ & $\begin{array}{r}1,000 \\
1,470 \\
\cdots 600 \\
600 \\
2,100\end{array}$ & $\begin{array}{l}34,300 \\
27,849 \\
15,000 \\
22,905 \\
12,000\end{array}$ & $\begin{array}{l}66 . \\
67 . \\
68 . \\
69 . \\
70 .\end{array}$ \\
\hline $\begin{array}{r}23,250 \\
11,867 \\
4,047 \\
70\end{array}$ & $\begin{array}{r}14,070 \\
5,491 \\
2,400 \\
69\end{array}$ & $\begin{array}{r}2,277 \\
577 \\
40 \\
67\end{array}$ & $\begin{array}{r}14,815 \\
5,910 \\
2,485 \\
70\end{array}$ & $\begin{array}{r}3,755 \\
661 \\
120 \\
65\end{array}$ & $\begin{array}{r}32,805 \\
19,382 \\
6,966 \\
70\end{array}$ & $\begin{array}{r}155.02 \\
32.07 \\
16.30 \\
69\end{array}$ & $\begin{array}{r}1,235,526 \\
455,493 \\
157,231 \\
65\end{array}$ & $\begin{array}{r}7.26 \\
4.17 \\
1.64 \\
65\end{array}$ & $\begin{array}{r}24,850 \\
11,965 \\
3,520 \\
69\end{array}$ & $\begin{array}{r}12,780 \\
6,000 \\
2,300 \\
69\end{array}$ & $\begin{array}{r}5,650 \\
840 \\
100 \\
62\end{array}$ & $\begin{array}{r}34,300 \\
18,395 \\
6,020 \\
69\end{array}$ & $\begin{array}{r}\text { High } \\
\text { Median } \\
\text { Low } \\
N^{5}\end{array}$ \\
\hline
\end{tabular}

locuments. e Bibliographical count, does not include documents. $f$ Bibliographical count, only reported. g Documents included, no physical or bibliographical ount reported. $\mathbf{h}$ Documents not included, no physical or bibliographical count reported. i Includes non-periodical serials. $\boldsymbol{j}$ Does not include non-periodical erials. $\mathbf{k}$ Information $(a-j)$ not reported. 


\section{College and University Library Salary Statistics $1954-55$}

1. Alabama College

2. Alma (Mich.)

3. Anderson (Ind)

5. Ashland (Ohio)

6. Augustana (S.D.)

7. Aurora (Ill.)

8. Bard (N.Y.)

10. Beaver (Pa.)

11. Bennington ( $\mathrm{Vt}$.)

12. Bethany (Kan.)

13. Birmingham-Southe $n$. . . . . . . . . $\ldots \ldots \ldots \ldots \ldots \ldots$

14. Carroll (Wis.

15. Cedar Crest (Pa.).

16. Colorado College

18. Concordia (Minn )

18. Concordia (Min

19. Davidson (N.C)

21. Earlham (Ind.)

23. Emira \& Henry (Va.

24. Evansville (Ind.)

25. Findlay (Ohio).

26. Geneva (Pa.).

28. Grinnell (Iowa)

29. Guilford (N.C)

30. Gustavus Adolphus (Minn)

31. Hillsdale (Mich.).

32. Hiram (Ohio)

33. Hollins (Va.)

35. Houghton (N.Y.)

36. Huntington (Ind.)

38. Illinois Wesleyan.

39. Immaculate Heart

41. Juniata (Pa.)

42. Lawrence (Wis.)

43. Lebanon Valley (Pa.)

44. Linfield (Ore.)

45. Luther (lowa)

46. Manchester (Ind

48. Millsaps (Miss.)

49. Morningside (Iowa)

50. Mount Union (Ohio)

51. Nebraska Wesleyan

52. New Mexico Highlands.

53. Parsons (Iowa)

54. Randolph-Macon ( $\mathrm{Va}$ )

55. Ripon (Wis.)

56. Rosary (Ill.) . . . . .

57. Seneca, Coll. of the
58. Seton Hill (Pa.).

59. Springfield (Mass.)

60. Sweet Briar (Va.)

61. Talladega (Ala.)

62. Trinity (D.C.)

63. Union (Neb.)

63. Union ( $\mathrm{Neb}$.)

(Pa.) ..................... 4,700

65. Washington \& Jefferson $(\mathrm{Pa}.) \ldots \ldots \ldots \ldots \ldots \ldots \ldots \ldots \ldots \ldots \ldots \ldots, 4,800$

66. Washington \& Lee (Va.)

67. Wells (N.Y

68. Wiberforce (Ohio)

70. Wilmington (Ohio)

High. .

Low
Chief
Libraila

$\$ 4,900$
3,400
3,600
4,300
$\ldots .$.

3,600

5,000

3,400

3,700

3,500

4,500

5,324
2,800

5,415

3,700

5,000

3,780

3,900

3,300

3,812

4,620
4,500

$4,4 \mathrm{UU}$

$3,800^{2}$

5,005

4,100

3,739

4,000

4, 7 UU

3,600

3,800

5,650

3,900

5,500

3,500

$4,000^{1}$
$2,500^{16}$

3,800

$5,800^{15}$

4, 000

$4,720^{3}$
$2,250^{7}$

4,000

4,400
3,600

4,800

$3,450^{8}$

6,000

, 555

3,300

4,600
4,200

7,500

4,000

51
51

Salaries September 1, 195

Associate

Librarian

\$.....

3,300

4,000

3,400

4,000

3,900
3,200

i, $445^{\circ}$

.....

$\dddot{5}^{138}{ }^{11}$

......

3,600

$\ddot{2}, 700$

3,200

3,696

$\ldots{ }^{12}$

$\ldots$ 13 $^{13}$

$\cdots$

$\ddot{3}, 960$

$2,300^{3}$

…....

…..."

.....

2,900
3,800

3,600

3,700

3,300

$3,400^{5}$
3,900

3,900
2,200

2,200
$4,500^{15}$

2,600
$4,500^{3}$

$4,500^{3}$
3,050

$\dddot{3}, 300$

3,311

3,000

3,600

$3,220^{8}$

$\ddot{2}, 398$

3,633

......

1,850

5,138

3,356
1,850

1,850
28

\author{
Department Head
}

Minimum Maximum No.

$\$ 3,400$

$\$ 3,600$

$\cdots \cdots$

$\cdots \cdots$

$\cdots \cdots$

$3,9 j j$

$\cdots \cdots$

3,103

$3,04 j$

3,230

3,035

$\ldots$

$\ldots$

$\ldots .$.

$\ldots \cdots$

3,300

3,950

2,900

3,200

3,300

2,500

3,20

$3,0 \mathrm{~J}$

3,600

……

.....

$\ddot{2,808}$

$2,460^{4}$

$4,000^{3}$

3,150

$\ddot{3}, 800$

3,036

3,400

......

......

…

3,500

.......

……

3,500

3,150
2,500

15

3,600
$\cdots \cdots$

……
Head of

School or

Dept'l
Libraries

\$....

4,400

4,625

……

...

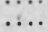

…
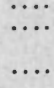

.....

$\ldots .$.

$\ldots$.

.....

.....

....

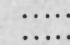

…

…

....

$\therefore$, J

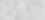

$\ldots$

……

$1 \$ 2,800-\$ 3,500$ (1). 2 For 10 months; summer school extra. 39 months. 410 months. 591 months. 6 Number o: libraries on which high. median (1). $12 \$ 3,000-\$ 3,600$ (2). $13 \$ 2,700-\$ 2,808$ (2). $14 \$ 3,150-\$ 5,050$ (I). 15 Part charged to teaching account. 16 Part-time. 


\section{(As of September I, I954) (Group III)}

Salaries September 1, 1954

\begin{tabular}{|c|c|c|c|c|c|c|c|c|c|c|c|}
\hline $\begin{array}{r}\text { si } \\
\text { Mini- } \\
\text { num }\end{array}$ & $\begin{array}{l}\text { ther Prof } \\
\text { Assista } \\
\text { Maxi- } \\
\text { mum }\end{array}$ & No. & $\begin{array}{l}\text { Mini- } \\
\text { mum }\end{array}$ & $\begin{array}{l}\text { Non-profe } \\
\text { Assistar } \\
\text { Maxi- } \\
\text { mum }\end{array}$ & No. & 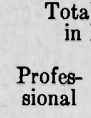 & $\begin{array}{c}\text { mber of } \\
\text { Time Eq } \\
\text { Non- } \\
\text { profes- } \\
\text { sional }\end{array}$ & $\begin{array}{l}\text { yees } \\
\text { nt }\end{array}$ & $\begin{array}{l}\text { Hours } \\
\text { in } \\
\text { Work } \\
\text { Week }\end{array}$ & $\begin{array}{c}\text { Hours } \\
\text { Student } \\
\text { Assist- } \\
\text { ance }\end{array}$ & \\
\hline $\begin{array}{c}\ldots \ldots \\
\ldots \ldots \\
\ldots \ldots \\
\ldots \ldots \\
\ldots \ldots\end{array}$ & $\begin{array}{c}\$ \ldots \\
\ldots \ldots \\
\ldots \ldots \\
2,500\end{array}$ & $\begin{array}{l}\because \\
\cdots \\
\cdots \\
i\end{array}$ & $\begin{array}{c}\$ \ldots \\
1,950 \\
\ldots \ldots \\
\ldots \ldots\end{array}$ & $\begin{array}{c}\ldots \ldots \\
\ldots \ldots \\
\ldots \ldots \\
2,500\end{array}$ & $\begin{array}{l}\ddot{1} \\
\because \\
i\end{array}$ & $\begin{array}{l}4 \\
1 \\
3 \\
2 \\
1.75\end{array}$ & $\begin{array}{l}.88 \\
.5 \\
1\end{array}$ & $\begin{array}{l}4 \\
1.88 \\
3.5 \\
2 \\
2.75\end{array}$ & $\begin{array}{l}40 \\
40 \\
40 \\
42 \\
40\end{array}$ & $\begin{array}{l}5,075 \\
1,147 \\
4,192 \\
4,225 \\
\ldots \ldots\end{array}$ & 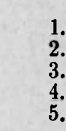 \\
\hline $\begin{array}{c}3,600 \\
3,300 \\
3,150 \\
\ldots \ldots\end{array}$ & $\begin{array}{l}\ldots \ldots \\
3,300 \\
\ldots \ldots \\
\ldots \ldots\end{array}$ & $\begin{array}{l}1 \\
1 \\
1 \\
\cdots\end{array}$ & $\begin{array}{l}3,200 \\
2,000 \\
2,100 \\
2,200\end{array}$ & $\begin{array}{l}\ldots \ldots \\
\cdots \cdots \\
\cdots \cdots \\
\cdots \cdots \\
\cdots \cdots\end{array}$ & $\begin{array}{l}1.33 \\
3 \\
1 \\
\cdots\end{array}$ & $\begin{array}{l}2 \\
1.33 \\
3 \\
4 \\
2.75\end{array}$ & $\begin{array}{l}1.5 \\
2.5 \\
1 \\
\ldots .\end{array}$ & $\begin{array}{l}2 \\
2.83 \\
5.5 \\
5 \\
2.75\end{array}$ & $\begin{array}{l}40 \\
40 \\
38 \\
39 \\
40\end{array}$ & $\begin{array}{l}5,000 \\
3,316 \\
4,195 \\
3,833 \\
\ldots \ldots\end{array}$ & 10 \\
\hline $\begin{array}{l}\ldots \ldots \\
\cdots \cdots \\
\cdots \cdots \\
\cdots \cdots \\
\cdots \cdots\end{array}$ & $\begin{array}{l}\ldots \ldots \\
\ldots \ldots \\
\ldots \ldots \\
\ldots \ldots \\
\cdots \cdots\end{array}$ & $\begin{array}{l}\ldots \\
\cdots \\
\cdots \\
\cdots\end{array}$ & $\begin{array}{l}1,473 \\
2,265 \\
\ldots \ldots \\
\ldots \ldots\end{array}$ & $\begin{array}{l}\ldots \ldots \\
\ldots \ldots \\
\ldots \ldots \\
\ldots \ldots \\
\ldots \ldots\end{array}$ & $\begin{array}{l}\cdots \\
\cdots \\
\cdots \\
\cdots\end{array}$ & $\begin{array}{l}3 \\
1 \\
3 \\
1.5 \\
1\end{array}$ & $\begin{array}{l}1 \\
6 \\
1 \\
.5 \\
.5\end{array}$ & $\begin{array}{l}4 \\
7 \\
4 \\
2 \\
1.5\end{array}$ & $\begin{array}{l}39 \\
39 \\
39 \\
40\end{array}$ & $\begin{array}{r}995 \\
4,752 \\
5,857 \\
3,545 \\
860\end{array}$ & $\begin{array}{l}11 \\
12 \\
13 \\
14 \\
15\end{array}$ \\
\hline $\begin{array}{l}\ldots \ldots \\
2,500 \\
\ldots \ldots \\
\ldots \ldots\end{array}$ & $\begin{array}{l}\ldots \ldots \\
\cdots \cdots \\
\cdots \cdots \\
\cdots \cdots \\
\cdots \cdots\end{array}$ & $\begin{array}{c}\cdots \\
\cdots \\
\cdots \\
\cdots\end{array}$ & $\begin{array}{l}1,800 \\
2,200 \\
1,320\end{array}$ & $\begin{array}{l}2,000 \\
\ldots \ldots \\
\ldots \ldots \\
1,920\end{array}$ & $\begin{array}{l}4 \\
1 \\
2\end{array}$ & $\begin{array}{l}4 \\
3 \\
2 \\
3.5 \\
2\end{array}$ & $\begin{array}{l}3.5 \\
1 \\
2 \\
2\end{array}$ & $\begin{array}{l}7.5 \\
3 \\
3 \\
3.5 \\
4\end{array}$ & $\begin{array}{l}39 \\
42 \\
39 \\
40\end{array}$ & $\begin{array}{l}4,494 \\
3,487 \\
3,932 \\
4,800 \\
3,286\end{array}$ & $\begin{array}{l}16 \\
17 \\
18 \\
19 \\
20\end{array}$ \\
\hline $\begin{array}{l}2,500 \\
\cdots \cdots \\
\cdots \cdots \\
\cdots \cdots \\
\cdots \cdots\end{array}$ & $\begin{array}{l}3,200 \\
\cdots \cdots \\
\cdots \cdots \\
\cdots \cdots \\
\cdots \cdots\end{array}$ & $\begin{array}{l}1 \\
\cdots \\
\cdots \\
\cdots\end{array}$ & $\begin{array}{l}1,300 \\
\ldots \ldots \\
2,100 \\
\ldots \ldots\end{array}$ & $\begin{array}{l}2,000 \\
\ldots \ldots \\
\ldots \ldots \\
\ldots \ldots \\
\cdots\end{array}$ & $\begin{array}{l}1 \\
\cdots \\
1 \\
\cdots\end{array}$ & $\begin{array}{l}2 \\
1 \\
2 \\
2 \\
1\end{array}$ & $\begin{array}{l}2 \\
2 \\
\ldots \\
1 \\
\ldots\end{array}$ & $\begin{array}{l}4 \\
3 \\
2 \\
3 \\
1\end{array}$ & $\begin{array}{l}40 \\
39 \\
40 \\
46.5\end{array}$ & $\begin{array}{l}3,679 \\
2,464 \\
5,518 \\
2,100 \\
1,056\end{array}$ & $\begin{array}{l}21 . \\
22 . \\
23 . \\
24 . \\
25 .\end{array}$ \\
\hline $\begin{array}{l}\ldots \ldots \\
2,500^{3} \\
\ldots \ldots \\
\cdots \cdots\end{array}$ & $\begin{array}{l}\ldots \ldots \\
3,800 \\
\ldots \ldots \\
\ldots \ldots\end{array}$ & $\begin{array}{l}. . \\
\cdots \\
\cdots \\
\cdots\end{array}$ & $\begin{array}{l}1,575^{3} \\
1,800\end{array}$ & $\begin{array}{l}\cdots \ldots \\
2,200 \\
2,280\end{array}$ & $\begin{array}{l}\cdots \\
\cdots \\
\cdots \\
1.5\end{array}$ & $\begin{array}{l}2.5 \\
3 \\
4.75 \\
3.5 \\
3\end{array}$ & $\begin{array}{l}1 \\
2 \\
1.75 \\
.5 \\
1.5\end{array}$ & $\begin{array}{l}3.5 \\
5 \\
6.5 \\
4 \\
4.5\end{array}$ & $\begin{array}{l}36 \\
40 \\
40 \\
40\end{array}$ & $\begin{array}{l}1,901 \\
2,676 \\
1,215 \\
3,281\end{array}$ & $\begin{array}{l}26 \\
27 \\
28 \\
29 \\
30\end{array}$ \\
\hline $\begin{array}{l}\ldots \ldots \\
2,400 \\
\ldots \ldots \\
\ldots \ldots\end{array}$ & $\begin{array}{l}\ldots \ldots \\
\ldots \ldots \\
\ldots \ldots \\
\ldots \ldots \\
\cdots \cdots\end{array}$ & $\begin{array}{l}\ldots \\
\cdots \\
\cdots \\
\cdots\end{array}$ & $\begin{array}{l}1,500 \\
\ldots \ldots \\
\cdots \cdots \\
\cdots \cdots \\
\cdots \cdots\end{array}$ & $\begin{array}{l}\ldots \ldots \\
\cdots \cdots \\
\cdots \cdots \\
\cdots \cdots \\
\cdots \cdots\end{array}$ & $\begin{array}{l}1 \\
\cdots \\
\cdots \\
\cdots\end{array}$ & $\begin{array}{l}1 \\
3 \\
3 \\
3 \\
2\end{array}$ & $\begin{array}{l}1 \\
1.5 \\
2^{.57}\end{array}$ & $\begin{array}{l}2 \\
4.5 \\
3 \\
3.57 \\
4\end{array}$ & $\begin{array}{l}40 \\
40 \\
36 \\
44\end{array}$ & $\begin{array}{r}500 \\
2,240 \\
1880 \\
5,083 \\
\ldots \ldots\end{array}$ & $\begin{array}{l}31 . \\
32 . \\
33 . \\
34 . \\
35 .\end{array}$ \\
\hline $\begin{array}{l}3,400 \\
\ldots \ldots \\
\ldots \ldots \\
\cdots \cdots\end{array}$ & $\begin{array}{l}\ldots \ldots \\
\ldots \ldots \\
\cdots \cdots \\
\cdots \cdots \\
\cdots \cdots\end{array}$ & $\begin{array}{l}\cdots \\
\cdots \\
\cdots \\
\cdots\end{array}$ & $\begin{array}{l}1,000 \\
2,080 \\
3,300 \\
\ldots \ldots\end{array}$ & $\begin{array}{l}\ldots \ldots \\
\ldots \ldots \\
3,600 \\
\ldots \ldots\end{array}$ & $\begin{array}{l}\cdots \\
\cdots \\
1 \\
\cdots\end{array}$ & $\begin{array}{l}1 \\
1.5 \\
2.5 \\
4 \\
1\end{array}$ & $\begin{array}{r}.5 \\
1.5 \\
1.5\end{array}$ & $\begin{array}{l}1 \\
2 \\
4 \\
5 \\
1.5\end{array}$ & $\begin{array}{l}40 \\
38 \\
40 \\
40 \\
\ldots\end{array}$ & \begin{tabular}{l}
1,963 \\
1,900 \\
6,240 \\
\hdashline, 326
\end{tabular} & $\begin{array}{l}36 . \\
37 . \\
38 . \\
39 . \\
40 .\end{array}$ \\
\hline $\begin{array}{l}\ldots \ldots \\
\ldots \ldots \\
1,500 \\
\ldots \ldots\end{array}$ & $\begin{array}{l}\ldots \ldots \\
\ldots \ldots \\
\ldots \ldots \\
\ldots \ldots \\
\ldots \ldots\end{array}$ & $\begin{array}{l}\ldots \\
\cdots \\
\cdots \\
\cdots\end{array}$ & $\begin{array}{l}2,000 \\
\ldots \ldots \\
1,500\end{array}$ & $\begin{array}{c}2,100 \\
\ldots \ldots \\
\ldots \ldots \\
3,000\end{array}$ & $\begin{array}{l}2 \\
2 \\
\because \\
2\end{array}$ & $\begin{array}{l}2 \\
3 \\
4 \\
2 \\
3\end{array}$ & $\begin{array}{l}2^{.33} \\
\ldots \\
2^{\cdots}\end{array}$ & $\begin{array}{l}2.33 \\
5 \\
4 \\
2 \\
5\end{array}$ & $\begin{array}{l}40 \\
40 \\
38 \\
40 \\
39\end{array}$ & \begin{tabular}{l}
3,700 \\
1,700 \\
1,783 \\
\hdashline 7,000
\end{tabular} & $\begin{array}{l}41 . \\
42 . \\
43 . \\
44 . \\
45 .\end{array}$ \\
\hline $\begin{array}{l}\ldots \ldots \\
2,700 \\
\ldots \ldots \\
\ldots \ldots\end{array}$ & $\begin{array}{l}\ldots \ldots \\
\ldots \ldots \\
\cdots \cdots \\
\cdots \cdots \\
\cdots \cdots\end{array}$ & $\begin{array}{l}\cdots \\
i \\
\cdots \\
\cdots\end{array}$ & $\begin{array}{l}\ldots \ldots \\
\ldots \ldots \\
\cdots \cdots \\
1,900^{8}\end{array}$ & $\begin{array}{l}\ldots \\
\ldots \\
\ldots \\
\ldots \\
\ldots\end{array}$ & $\begin{array}{l}\cdots \\
\cdots \\
\cdots \\
1\end{array}$ & $\begin{array}{l}2 \\
2 \\
3.5 \\
2 \\
4\end{array}$ & $\begin{array}{l}\ldots \\
\cdots \\
\cdots \\
1\end{array}$ & $\begin{array}{l}2 \\
2 \\
3.5 \\
2 \\
5\end{array}$ & $\begin{array}{l}40 \\
40 \\
40 \\
37.5\end{array}$ & $\begin{array}{l}4,976 \\
3,300 \\
1,480 \\
3,528 \\
4,000\end{array}$ & $\begin{array}{l}46 . \\
47 . \\
48 . \\
49 . \\
50 .\end{array}$ \\
\hline $\begin{array}{l}\ldots \ldots \\
\ldots \ldots \\
\cdots \cdots \\
\cdots \cdots \\
\cdots \cdots\end{array}$ & $\begin{array}{l}\ldots \ldots \\
\ldots \ldots \\
\cdots \cdots \\
\cdots \cdots \\
\cdots \cdots\end{array}$ & $\begin{array}{l}\cdots \\
\cdots \\
\cdots \\
\cdots\end{array}$ & $\begin{array}{l}\cdots \cdots \\
\cdots \\
1,250 \\
2,100\end{array}$ & $\begin{array}{l}\ldots \ldots \\
\ldots \ldots \\
1,800 \\
\ldots \ldots\end{array}$ & $\begin{array}{l}\cdots \\
\cdots \\
3 \\
1\end{array}$ & $\begin{array}{l}1 \\
3 \\
1 \\
4 \\
2\end{array}$ & $\begin{array}{l}\cdots \\
1.5 \\
3 \\
1\end{array}$ & $\begin{array}{l}1 \\
3 \\
2.5 \\
7 \\
3\end{array}$ & $\begin{array}{l}40 \\
38 \\
43 \\
38 \\
40\end{array}$ & $\begin{array}{l}7,310 \\
1,075 \\
\ldots \ldots \\
\ldots \ldots\end{array}$ & $\begin{array}{l}51 . \\
52 . \\
53 . \\
54 . \\
55 .\end{array}$ \\
\hline $\begin{array}{l}\therefore \ldots \\
2,500 \\
3,000\end{array}$ & $\begin{array}{l}\ldots \ldots \\
\ldots \cdots \\
\cdots \cdots \\
\cdots \cdots \\
\cdots \cdots\end{array}$ & $\begin{array}{l}\cdots \\
\cdots \\
i\end{array}$ & $\begin{array}{l}1,950 \\
\ldots \ldots \\
1,600\end{array}$ & $\begin{array}{l}2,340 \\
\ldots \ldots \\
2,075\end{array}$ & $\begin{array}{l}2 \\
\cdots \\
3\end{array}$ & $\begin{array}{l}3 \\
3 \\
2 \\
4\end{array}$ & $\begin{array}{l}3.65 \\
3.5 \\
3\end{array}$ & $\begin{array}{l}6.65 \\
3 \\
5.5 \\
7\end{array}$ & $\begin{array}{l}37.5 \\
40 \\
39\end{array}$ & $\begin{array}{r}808 \\
3,046 \\
2,250 \\
2,431 \\
1,263\end{array}$ & $\begin{array}{l}56 . \\
57 . \\
58 . \\
59 . \\
60 .\end{array}$ \\
\hline $\begin{array}{l}3,300 \\
\ldots \ldots \\
\ddot{2}, 500\end{array}$ & $\begin{array}{l}3,600 \\
\ldots \ldots \\
\ldots \ldots \\
\ldots \ldots\end{array}$ & $\begin{array}{l}2 \\
\cdots \\
1\end{array}$ & $\begin{array}{l}1,468^{8} \\
\ldots \ldots \\
2,250 \\
1,820\end{array}$ & $\begin{array}{l}2,185^{8} \\
\ldots \ldots \\
\ldots \ldots \\
\ldots \ldots \\
\ldots \ldots\end{array}$ & $\begin{array}{l}2 \\
\cdots \\
\cdots \\
i\end{array}$ & $\begin{array}{l}2 \\
2.75 \\
2 \\
2 \\
2\end{array}$ & $\begin{array}{l}2 \\
.25 \\
\cdots .67 \\
1\end{array}$ & $\begin{array}{l}4 \\
3 \\
2 \\
2.67 \\
3\end{array}$ & $\begin{array}{l}37 \\
39 \\
38 \\
40\end{array}$ & $\begin{array}{r}2,273 \\
3,605 \\
10,000 \\
2,750 \\
2,090\end{array}$ & $\begin{array}{l}61 . \\
62 . \\
63 . \\
64 . \\
65 .\end{array}$ \\
\hline $\begin{array}{l}3,350 \\
\ldots \ldots \\
\ldots \ldots \\
\ldots \ldots\end{array}$ & $\begin{array}{l}\ldots \ldots \\
\ldots \ldots \\
\ldots \ldots \\
\ldots \ldots \\
\ldots \ldots\end{array}$ & $\begin{array}{l}\ddot{2} \\
\cdots \\
\cdots \\
\cdots\end{array}$ & $\begin{array}{l}1,584 \\
2,400 \\
2,0 j 0 \\
\ldots \ldots\end{array}$ & $\begin{array}{l}2,436 \\
\ldots \ldots \\
\cdots \cdots \\
\ldots \ldots \\
\ldots \cdots\end{array}$ & $\begin{array}{l}3.5 \\
2 \\
\cdots \\
\cdots \\
\cdots\end{array}$ & $\begin{array}{l}3 \\
3 \\
2 \\
3 \\
1.5\end{array}$ & $\begin{array}{l}3.5 \\
2 \\
\ldots \\
1 \\
.5\end{array}$ & $\begin{array}{l}6.5 \\
5 \\
2 \\
4 \\
2\end{array}$ & $\begin{array}{l}38 \\
38 \\
40 \\
40 \\
38\end{array}$ & $\begin{array}{r}710 \\
6,000 \\
4,155 \\
2,600\end{array}$ & $\begin{array}{l}66 . \\
67 . \\
68 . \\
69 . \\
70 .\end{array}$ \\
\hline $\begin{array}{r}3,600 \\
2,850 \\
1,500 \\
14\end{array}$ & $\begin{array}{r}3,800 \\
3,300 \\
2,500 \\
5\end{array}$ & $\begin{array}{r}2 \\
1 \\
1 \\
11\end{array}$ & $\begin{array}{r}3,300 \\
1,975 \\
1,000 \\
28\end{array}$ & $\begin{array}{r}3,600 \\
2,190 \\
1,800 \\
12\end{array}$ & 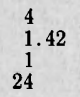 & $\begin{array}{r}4 \\
2 \\
1 \\
69\end{array}$ & $\begin{array}{c}6 \\
1 \\
48^{.25}\end{array}$ & $\begin{array}{l}7.5 \\
3 \\
1 \\
69\end{array}$ & $\begin{array}{l}46.5 \\
40 \\
36 \\
60\end{array}$ & $\begin{array}{r}10,000 \\
3,284 \\
500 \\
60\end{array}$ & $\begin{array}{r}\text { High } \\
\text { Median } \\
\text { Low } \\
\text { No }^{\circ}\end{array}$ \\
\hline
\end{tabular}




\section{Teachers College Library}

\section{Library}

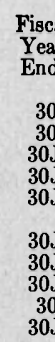

Fiscal
Year
Endin

30S
30S
30Je
30Je
30Je

30J
30Je
30Je
30
30J

11. Illinois, Charleston, Eastern State College.

12. Illinois, Dekalb, Northern State Teachers College

13. Indiana, Terre Haute, State Teachers College

14. Iowa, Cedar Falls, State Teachers College

15. Kansas, Emporia, State Teachers College . . . . . . . . . . . .

16. Kansas, Pittsburg, State Teachers College................ 30Je54

17. Kentucky, Murray, State College

18. Louisiana, Natchitoches, Northwestern State College.

19. Maryland, Frostburg, State Teachers College.

20. Maryland, Towson, State Teachers College

21. Massachusetts, North Adams, State Teachers College

22. Michigan, Kalamazoo, Western Michigan College

23. Michigan, Marquette, Northern Michigan College of Education.. 24. Michigan, Ypsilanti, Michigan State Normal College

25. Minnesota, Bemidji, State Teachers College.

26. Minnesota, Mankato, State Teachers College.

27. Minnesota, Moorhead, State Teachers College

28. Minnesota, St. Cloud, State Teachers College

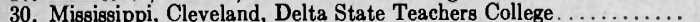

31. Missouri, Cape Girardeau, Southeast State College ........... 30Ap54

32. Missouri, Springfield, Southwest State College.............. 30 Je5

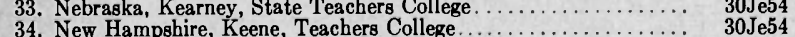

34. New Hampshire, Keene, Teachers College $\ldots \ldots \ldots \ldots \ldots \ldots \ldots \ldots$
35. New Jersey, Glissboro, State Teachers College............... 30 3e54

36. New Jersey, Paterson, State Teachers College.............. 30. Je54 37. New Jersey, Trenton, State Teachers College $\ldots \ldots \ldots \ldots \ldots \ldots \ldots \ldots$ 30Je54 38. New York, Albany, State Teachers College ............... 31Mr54 39. New York, Oswego, State University Teachers College........ 31Mr54 40. North Carolina, Boone, Appalachine State Teachers College..... 30Je54

41. North Carolina, Greenville, East Carolina College............ 30Je54 42. Oklahoma, Alva, Northwestern State College............. 30Je54 43. Oregon, Ashland, Southern College of Education.............. 30Je54 44. Oregon, La Grande, Eastern College of Education ..............
45. Oregon, Monmouth, College of Education................

46. Pennsylvania, Bloomsburg, State Teachers College........... 31My54 47. Pennsylvania, California, State Teachers College ............. $31 \mathrm{My54}$ 48. Pennsylvania, Clarion, State Teachers College ............ $31 \mathrm{My54}$ 49. Pennsylvania, Kutztown, State Teachers College.............. $31 \mathrm{My54}$

51. Pennsylvania, West Chester, State Teachers College. 54. Texas, San Marcos Southwest State Teachers College 55. Virginia, Farmville, Longwood College... $31 \mathrm{Au} 54$ 30Je54

Under

Gradu933

899
1,137

605
1,478

3,163

2,393

4,007

1,640

476
1,717

30Je54 1,002

30Je5

$30 \mathrm{Je5}$

$30 \mathrm{Je} 54$

57. Virginia, Petersburg, State College.

58. West Virginia, Fairmont, State College

61. Wisconsin, Oshkosh, State College

$30 \mathrm{Je} 54$

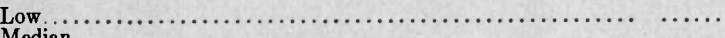

High.
1,350

1,350
2,032
1,715

2,141

1,036

1,585

1,450

1,401

959

141

4,005
581

581
2,285
497

1,681

601
1,255

481
437

1,040

1,621
622

469

530

856
1,505

1,179

1,139

2,055

460

550
460

467

727

655

420

597

1,658

2,007

1,609
1,430

653

1,481

688

1,850

736

141

83,999

93,021

167,959
106,087

102,715

50,474

70,116

46,851

12,399

100,295

52,767

112,441
32,949

47,527

39,953
77,304

39,000

35,274

77,000

83,688

42,808

24,117
37,127

22,196

71,828

56,812

60,746

90,840

36,813

29,140

27,862
33,289

37,022

27,559

29,210

44,439
34,712

63,739

120,946

120,946
123,106

123,106
80,255

61,437

71,837

59,166

40,821

79,029

51,899

12,399

1,002
4,007

3
126
2,082
Book

V

$0 \quad 34,310$

34,310
39,079

39,079
56,136

48,365

64,116

99,613

1, 082

49,032

100,843

141,772

$\begin{array}{ccc}\text { Volumes } & \text { News- } & \begin{array}{c}\text { Periodi- } \\ \text { Added }\end{array} \\ \text { papers } & \end{array}$

$\begin{array}{lll}1,517 & 8 & 215\end{array}$

$\begin{array}{lll}1,704 & 10 & 264 \\ 4,073 & 11 & 262\end{array}$

$\begin{array}{lll}6,213 & 11 & 262 \\ 4,828 & 21 & 579\end{array}$

4,828

9,230

12,482
8,247

4, 016

1,973

4,224

7,370
3,601

3,601
5,514

3,286

3,920

1,640

1,352

1,352
3,779

446

4,867

1,774
3,640

3,640
1,698

5,173

1,960
5,611

1,181

2,153

2,000

1,714

1,034
1,543

1,050

1,639

3,200

3,776
3,757

3,398

4,373

2,204

3,623
1,831

2,609

1,140

1,659

1,162

1,226

2,253

3,114

7,48

2,365
2,124

4,160

2,767

1,596

2,832

1,746

446 


\begin{tabular}{|c|c|c|c|c|c|c|c|c|c|c|c|}
\hline $\begin{array}{c}\text { Staff } \\
\text { Salaries }\end{array}$ & $\begin{array}{l}\text { Student } \\
\text { Service }\end{array}$ & $\begin{array}{l}\text { Total } \\
\text { Staff } \\
\text { Salaries } \\
\text { and } \\
\text { Student } \\
\text { Service }\end{array}$ & Books & Binding & $\begin{array}{l}\text { Total } \\
\text { Books } \\
\text { Periodi- } \\
\text { cals } \\
\text { Binding }\end{array}$ & $\begin{array}{l}\text { Other } \\
\text { Operat- } \\
\text { ing } \\
\text { Expendi- } \\
\text { tures }\end{array}$ & $\begin{array}{l}\text { Total } \\
\text { Operat- } \\
\text { ing } \\
\text { Expendi- } \\
\text { tures }\end{array}$ & $\begin{array}{l}\text { Operat- } \\
\text { ing } \\
\text { Expendi- } \\
\text { tures } \\
\text { per } \\
\text { student }\end{array}$ & $\begin{array}{l}\text { Total } \\
\text { Institu- } \\
\text { tional } \\
\text { Expendi- } \\
\text { tures }\end{array}$ & $\begin{array}{l}\text { Per Cent } \\
\text { of Total } \\
\text { Expendi- } \\
\text { tures for } \\
\text { Library } \\
\text { Purposes }\end{array}$ & \\
\hline $\begin{array}{r}\mathbf{7}, 696.54 \\
8,244 . \tilde{U}^{-1} \\
19,424.64 \\
45,110.00 \\
45,396.00\end{array}$ & $\begin{array}{r}3,167.25 \\
4,298.00 \\
3,599.00 \\
4,700.00 \\
8,700.00\end{array}$ & $\begin{array}{l}10,863.79 \\
12,542.00 \\
23,023.64 \\
49,810.00 \\
54,096.00\end{array}$ & $\begin{array}{r}6,111.59 \\
7,666.67 \\
10,883.58 \\
20,100.00 \\
18,466.00\end{array}$ & $\begin{array}{r}623.95 \\
565.35 \\
295.92 \\
1,950.00 \\
2,520.00\end{array}$ & $\begin{array}{r}\$ 6,735.44 \\
8,232.02 \\
11,179.50 \\
22,050.00 \\
20,986.00\end{array}$ & $\begin{array}{r}655.46 \\
480.15 \\
1,455.90 \\
2,147.00 \\
3,159.00\end{array}$ & $\begin{array}{r}18,254.79 \\
27,254.17 \\
35,659.04 \\
74,007.00 \\
78,241.00\end{array}$ & $\begin{array}{r}19.56 \\
23.64 \\
31.36 \\
116.36 \\
50.44\end{array}$ & $\begin{array}{r}559,670.71 \\
549,632.67 \\
798,521.78 \\
848,994.00 \\
1,246,039.00\end{array}$ & $\begin{array}{l}3.24 \\
3.9 \\
4.47 \\
8.00 \\
6.27\end{array}$ & $\begin{array}{l}1 . \\
2 . \\
3 . \\
4 . \\
5 .\end{array}$ \\
\hline $\begin{array}{r}75,286.00 \\
47,784.00 \\
119,492.00 \\
36,390.00 \\
21,003.00\end{array}$ & $\begin{array}{r}12,891.00 \\
7,200.00 \\
15,658.00 \\
12,293.00 \\
2,947.00\end{array}$ & $\begin{array}{r}88,177.00 \\
54,984.00 \\
135,150.00 \\
48,683.00 \\
24,050.00\end{array}$ & $\begin{array}{r}30,042.00 \\
37,550.00 \\
38,100.00 \\
11,985.00 \\
4,829.00\end{array}$ & $\begin{array}{l}3,657.00 \\
4,400.00 \\
3,911.00 \\
1,600.00 \\
1,752.00\end{array}$ & $\begin{array}{r}33,699.00 \\
41,950.00 \\
42,011.00 \\
13,585.00 \\
6,581.00\end{array}$ & $\begin{array}{r}9,382.00 \\
3,335.00 \\
4,601.00 \\
1,274.00 \\
194.00\end{array}$ & $\begin{array}{r}131,258.00 \\
100,269.00 \\
181,762.00 \\
63,542.00 \\
30,825.00\end{array}$ & $\begin{array}{l}36.07 \\
33.77 \\
29.85 \\
34.89 \\
56.00\end{array}$ & $\begin{array}{r}2,494,592.00 \\
1,299,397.49 \\
3,138,143.00 \\
1,412,682.00 \\
464,352.00\end{array}$ & $\begin{array}{l}5.26 \\
7.7 \\
5.79 \\
4.5 \\
6.6\end{array}$ & $\begin{array}{r}6 . \\
7 . \\
8 . \\
9 . \\
10 .\end{array}$ \\
\hline $\begin{array}{l}53,990.00 \\
57,260.65 \\
33,154.00 \\
38,980.00 \\
44,732.00\end{array}$ & $\begin{array}{r}6,247.00 \\
9,871.05 \\
2,874.00 \\
9,257.00 \\
11,932.00\end{array}$ & $\begin{array}{l}60.237 .00 \\
67,131.70 \\
36,028.00 \\
48,237.00 \\
56,644.00\end{array}$ & $\begin{array}{l}13,760.00 \\
16,237.86 \\
13,555.00 \\
25,504.00 \\
18,746.42\end{array}$ & $\begin{array}{l}1,982.00 \\
1,011.58 \\
2,172.00 \\
6,326.00 \\
1,278.00\end{array}$ & $\begin{array}{l}15,742.00 \\
17,249.44 \\
15,727.00 \\
31,830.00 \\
20,024.42\end{array}$ & $\begin{array}{r}3,175.00 \\
1,462.80 \\
1,351.00 \\
11,449.00 \\
3,586.00\end{array}$ & $\begin{array}{l}79,154.00 \\
85,843.94 \\
53,106.00 \\
91,516.00 \\
80,254.42\end{array}$ & $\begin{array}{l}\mathbf{5 8 . 0 0} \\
\mathbf{3 7 . 7 0} \\
28.00 \\
41.02 \\
\mathbf{7 1 . 0 6}\end{array}$ & $\begin{array}{l}1,393,255.00 \\
1,785,621.89 \\
1,603,473.00 \\
2,860,122.00 \\
1,316,443.00\end{array}$ & $\begin{array}{l}5.68 \\
4.8 \\
3.00 \\
3.2 \\
6.00\end{array}$ & $\begin{array}{l}11 . \\
12 . \\
13 . \\
14 . \\
15 .\end{array}$ \\
\hline $\begin{array}{l}28,296.45 \\
12,727.00 \\
38,624.39 \\
15,865.66 \\
25,122.67\end{array}$ & $\begin{array}{r}10,107.87 \\
3,200.00 \\
6,722.67 \\
802.76 \\
1,989.75\end{array}$ & $\begin{array}{l}38,404.32 \\
15,927.00 \\
45,347.06 \\
16,668.42 \\
27,112.42\end{array}$ & $\begin{array}{r}19,401.60 \\
9,525.00 \\
16,534.77 \\
5,204.58 \\
6,533.02\end{array}$ & $\begin{array}{r}1,836.41 \\
1,500.00 \\
2,300.00 \\
550.25 \\
4,537.58\end{array}$ & $\begin{array}{r}21,238.01 \\
11,025.00 \\
18,834.77 \\
5,754.83 \\
11,070.60\end{array}$ & $\begin{array}{r}24,104.27 \\
1,300.00 \\
3,393.33 \\
254.05 \\
2,268.51\end{array}$ & $\begin{array}{l}83,746 \cdot 60 \\
28,325 \cdot 00 \\
67,575 \cdot 16 \\
22,677 \cdot 30 \\
40,714.57\end{array}$ & $\begin{array}{l}48.24 \\
18.88 \\
48.23 \\
51.77 \\
42.20\end{array}$ & \begin{tabular}{c}
$1,333,248.04$ \\
\hdashline$, 393,522.32$ \\
$422,724.79$ \\
$768,917.51$
\end{tabular} & $\begin{array}{l}4.85 \\
5.36 \\
5.29\end{array}$ & $\begin{array}{l}16 . \\
17 . \\
18 . \\
19 . \\
20 .\end{array}$ \\
\hline $\begin{array}{r}5,100.00 \\
62,015.00 \\
16,300.00 \\
46,500.00 \\
11,856.00\end{array}$ & $\begin{array}{r}291.93 \\
11,887.44 \\
3,800.00 \\
8,750.00 \\
1,509.00\end{array}$ & $\begin{array}{r}5,391.93 \\
73,902.44 \\
20,150.00 \\
55,250.00 \\
13,365.00\end{array}$ & $\begin{array}{r}1,835.43 \\
15,316.61 \\
52.00 \\
12,065.00 \\
5,462.00\end{array}$ & $\begin{array}{r}0 \\
2,702.16 \\
1,000.00 \\
1,500.00 \\
706.00\end{array}$ & $\begin{array}{r}1,835.43 \\
18,018.77 \\
6,200.00 \\
13,565.00 \\
6,168.00\end{array}$ & $\begin{array}{r}167.22 \\
2,561.86 \\
700.00 \\
1,295.00 \\
175.00\end{array}$ & $\begin{array}{r}7,394.58 \\
94,483.07 \\
27,050.00 \\
70,110.00 \\
19,708.00\end{array}$ & $\begin{array}{l}34.23 \\
21.68 \\
46.55 \\
28.67 \\
39.65\end{array}$ & $\begin{array}{r}145,414.00 \\
2,897,685.00 \\
725.385 .00 \\
2,147,287.00 \\
490,754.00\end{array}$ & $\begin{array}{l}5.08 \\
3.26 \\
3.7 \\
3.2 \\
4.00\end{array}$ & $\begin{array}{l}21 . \\
22 . \\
23 . \\
24 . \\
25 .\end{array}$ \\
\hline $\begin{array}{l}21,714.00 \\
13,860.00 \\
29,404.25 \\
10,110.00 \\
14,633.30\end{array}$ & $\begin{array}{l}2,791.00 \\
1,452.13 \\
2,860.70 \\
2,169.00 \\
1,580.00\end{array}$ & $\begin{array}{l}24,505.00 \\
15,312.13 \\
32,265.05 \\
12,279.00 \\
16,213.30\end{array}$ & $\begin{array}{r}17,421.00 \\
7,648.95 \\
16,004.03 \\
4,000.00 \\
6,634.84\end{array}$ & $\begin{array}{r}801.00 \\
535.26 \\
3,522.15 \\
85.00 \\
1,045.02\end{array}$ & $\begin{array}{r}18,222.00 \\
8,184.21 \\
19,526.18 \\
4,085.00 \\
7,680.86\end{array}$ & $\begin{array}{r}1,215.00 \\
97.30 \\
1,434.94 \\
473.00 \\
69.73\end{array}$ & $\begin{array}{l}43,942.00 \\
23,593.64 \\
53,226.17 \\
16,837.00 \\
23,963.89\end{array}$ & $\begin{array}{l}25.52 \\
26.29 \\
42.17 \\
35.00 \\
54.83\end{array}$ & $\begin{array}{r}1,005,793.00 \\
562,288.15 \\
982,782.00 \\
431,161.00 \\
362,211.23\end{array}$ & $\begin{array}{l}4.37 \\
4.196 \\
5.41 \\
3.9 \\
6.00\end{array}$ & $\begin{array}{l}26 . \\
27 . \\
28 . \\
29 . \\
30 .\end{array}$ \\
\hline $\begin{array}{r}16,631.00 \\
22,309.00 \\
10,350.00 \\
7,670.00 \\
13,350.00\end{array}$ & $\begin{array}{r}3,607.00 \\
6,156.00 \\
3,755.00 \\
1,253.00 \\
956.30\end{array}$ & $\begin{array}{r}19,938.00 \\
28,465.00 \\
14,105.00 \\
8,923.00 \\
14,306.30\end{array}$ & $\begin{array}{r}11,971.00 \\
6,723.00 \\
5,330.00 \\
3,735.00 \\
2,430.45\end{array}$ & $\begin{array}{r}471.00 \\
777.00 \\
474.00 \\
139.00 \\
1,280.14\end{array}$ & $\begin{array}{r}12,442.00 \\
7,500.00 \\
5,804.00 \\
3,874.00 \\
4,710.59\end{array}$ & $\begin{array}{r}6,674.00 \\
0 \\
360.00 \\
463.00 \\
0\end{array}$ & $\begin{array}{l}39,054.00 \\
35,965.00 \\
20,269.00 \\
13,260.00 \\
19,016.89\end{array}$ & $\begin{array}{l}37.16 \\
22.10 \\
32.43 \\
28.27 \\
44.96\end{array}$ & $\begin{array}{l}645,460.00 \\
818,015.00 \\
540,661.00 \\
494,207.00 \\
422,918.91\end{array}$ & $\begin{array}{l}6.00 \\
4.00 \\
3.75 \\
2.68 \\
4.5\end{array}$ & $\begin{array}{l}31 . \\
32 . \\
33 . \\
34 . \\
35 .\end{array}$ \\
\hline $\begin{array}{l}17,720.00 \\
32,860.00 \\
44,946.00 \\
32,089.33 \\
24,235.00\end{array}$ & $\begin{array}{l}1,500.00 \\
3,162.00 \\
1,887.00 \\
1,404.50 \\
5,315.00\end{array}$ & $\begin{array}{l}19,220.00 \\
36,022.00 \\
46,833.00 \\
33,493.83 \\
29,550.00\end{array}$ & $\begin{array}{r}9,334.93 \\
7,703.00 \\
12,679.00 \\
10,511.36 \\
10,993.00\end{array}$ & $\begin{array}{r}149.38 \\
535.00 \\
2,182.00 \\
1,275.00 \\
1,585.00\end{array}$ & $\begin{array}{r}9,484.31 \\
8,238.00 \\
14,861.00 \\
11,786.36 \\
12,578.00\end{array}$ & $\begin{array}{r}120.00^{2} \\
2,163.00 \\
6,402.93 \\
0\end{array}$ & $\begin{array}{l}28,704.31 \\
44,380.00 \\
63,857.00 \\
51,683.12 \\
42,128.00\end{array}$ & $\begin{array}{l}54.16 \\
52.00 \\
38.00 \\
49.09 \\
36.22\end{array}$ & $\begin{array}{c}380,671.35 \\
585,078.00 \\
1,119,200.00^{3} \\
902,175.00 \\
596,172.00\end{array}$ & $\begin{array}{l}7.54 \\
7.58 \\
5.7 \\
6.00 \\
7.06\end{array}$ & $\begin{array}{l}36 . \\
37 . \\
38 . \\
39 . \\
40 .\end{array}$ \\
\hline $\begin{array}{r}40,114.43 \\
9,511.92 \\
19,674.00 \\
19,504.10 \\
9,770.00\end{array}$ & $\begin{array}{l}7,637.76 \\
1,201.00 \\
3,148.00 \\
2,847.00 \\
5,953.51\end{array}$ & $\begin{array}{l}47,752.19 \\
10,712.92 \\
22,822.00 \\
22,352.00 \\
15,723.51\end{array}$ & $\begin{array}{r}14,249.04 \\
6,867.60 \\
5,701.00 \\
6,707.00 \\
7,085.32\end{array}$ & $\begin{array}{r}1,999.59 \\
422.26 \\
545.00 \\
631.00 \\
617.07\end{array}$ & $\begin{array}{r}16,248.63 \\
7,289.86 \\
6,246.00 \\
7,348.00 \\
7,702.39\end{array}$ & $\begin{array}{r}1,594.88 \\
513.09 \\
10,289.00 \\
1,255.00 \\
1,840.08\end{array}$ & $\begin{array}{l}65,595.70 \\
18,516.57 \\
39,357.00 \\
30,955.00 \\
25,265.98\end{array}$ & $\begin{array}{l}29.64 \\
40.25 \\
71.17 \\
65.20 \\
54.10\end{array}$ & $\begin{array}{l}930,536.00 \\
411,761.52 \\
516,688.00 \\
396,221.00 \\
537,113.96\end{array}$ & $\begin{array}{l}7.15 \\
4.4 \\
7.6 \\
7.9 \\
4.00\end{array}$ & $\begin{array}{l}41 . \\
42 . \\
43 . \\
44 . \\
45 .\end{array}$ \\
\hline $\begin{array}{c}10,500.00 \\
9,775.00 \\
11,733.00 \\
13,840.00^{4} \\
14,672.53\end{array}$ & $\begin{array}{r}1,800.00 \\
1,812.00 \\
2,285.00 \\
2,614.00 \\
794.90\end{array}$ & $\begin{array}{l}12,300.00 \\
11,587.00 \\
14,018.00 \\
16,454.00 \\
15,467.43\end{array}$ & $\begin{array}{l}4,819.00 \\
4,657.00 \\
3,746.00 \\
7,000.00 \\
2,424.78\end{array}$ & $\begin{array}{l}700.00 \\
316.00 \\
103.00 \\
233.00 \\
134.90\end{array}$ & $\begin{array}{l}\mathbf{5}, 519.00 \\
4,973.00 \\
3,849.00 \\
7,233.00 \\
2,559.68\end{array}$ & $\begin{array}{l}675.00 \\
300.00 \\
203.00 \\
700.00 \\
476.10\end{array}$ & $\begin{array}{l}18,494.00 \\
16,860.00 \\
18,070.00 \\
24,387.00 \\
18,503.21\end{array}$ & $\begin{array}{l}25.00 \\
25.74 \\
43.00 \\
29.00 \\
30.99\end{array}$ & $\begin{array}{l}497,382.00 \\
535,887.00 \\
339,165.00 \\
521,051.00 \\
713,153.50\end{array}$ & $\begin{array}{l}4.00 \\
3.146 \\
5.3 \\
4.68 \\
2.00\end{array}$ & $\begin{array}{l}46 . \\
47 . \\
48 . \\
49 . \\
50 .\end{array}$ \\
\hline $\begin{array}{l}21,788.80 \\
23,218.67 \\
24,435.00 \\
22,552.50 \\
20,832.00\end{array}$ & $\begin{array}{r}4,275.00 \\
13,236.00 \\
2,580.58 \\
5,825.15 \\
2,298.00\end{array}$ & $\begin{array}{r}2,606,380.00 \\
36,454.67 \\
27,015.58 \\
28,377.65 \\
23,130.00\end{array}$ & $\begin{array}{r}3,273.25 \\
12,596.08 \\
11,018.14 \\
7,832.00 \\
5,663.60\end{array}$ & $\begin{array}{r}1,413.12 \\
746.78 \\
2,300.00 \\
991.91\end{array}$ & $\begin{array}{r}4,686.37 \\
12,596.08 \\
11,766.92 \\
10,132.00 \\
6,655.51\end{array}$ & $\begin{array}{r}2,183.63 \\
2,811.24 \\
1,785.31 \\
733.84 \\
593.14\end{array}$ & $\begin{array}{l}32,933.80 \\
51,861.99 \\
40,567.81 \\
39,243.49 \\
30,378.65\end{array}$ & $\begin{array}{l}19.86 \\
29.26 \\
22.31 \\
23.90 \\
46.52\end{array}$ & $\begin{array}{r}1,435,076.94 \\
1,151,392.66 \\
1,042,523.18 \\
963,187.00 \\
779,969.00\end{array}$ & $\begin{array}{l}2.29 \\
4.5 \\
3.86 \\
4.00 \\
3.8\end{array}$ & \\
\hline $\begin{array}{r}23,740.00 \\
34,327.00 \\
17,237.17 \\
8,218.65 \\
31,395.00\end{array}$ & $\begin{array}{l}2,805.00 \\
6,596.00 \\
1,816.25 \\
2,311.17 \\
3,942.00\end{array}$ & $\begin{array}{l}26,545 \cdot 00 \\
40,923 \cdot 00 \\
19,053.42 \\
10,529.82 \\
35,347.00\end{array}$ & $\begin{array}{r}11,190.00 \\
13,059.86 \\
9,122.48 \\
5,024.41 \\
13,843.00\end{array}$ & $\begin{array}{r}1,393.00 \\
1,440.00 \\
549.76 \\
420.98 \\
2,457.00\end{array}$ & $\begin{array}{r}12,583.00 \\
14,499.86 \\
9,672.24 \\
5,445.39 \\
16,300.00\end{array}$ & $\begin{array}{c}1,247.00 \\
1,151.82 \\
1,596.74 \\
600.00\end{array}$ & $\begin{array}{l}40,375.00 \\
55,422.86 \\
29,877.48 \\
17,571.95 \\
52,247.00\end{array}$ & $\begin{array}{l}40.28 \\
35.80 \\
43.42 \\
40.39 \\
24.30\end{array}$ & $\begin{array}{r}677,300.00 \\
2,102,226.00 \\
510,900.00 \\
327,708.64 \\
1,033,179.00\end{array}$ & $\begin{array}{l}5.96 \\
2.63 \\
5.8 \\
5.36 \\
5.05\end{array}$ & \\
\hline $17,375.00$ & $1,950.00$ & $19,325.00$ & $8,300.00$ & 630.00 & $8,930.00$ & 400.00 & $28,655.00$ & 38.93 & $552,700.00$ & 5.18 & 61. \\
\hline $\begin{array}{r}7,696.54 \\
21,714.00 \\
119,492.00\end{array}$ & $\begin{array}{r}802.76 \\
3,148.00 \\
15,658.00\end{array}$ & $\begin{array}{r}10,529.82 \\
24,505.00 \\
2,606,380.00\end{array}$ & $\begin{array}{r}52.00 \\
9,122.48 \\
38,100.00\end{array}$ & $\begin{array}{r}103.00 \\
1,000.00 \\
6,326.00\end{array}$ & $\begin{array}{r}2,559.68 \\
10,132.00 \\
42,011.00\end{array}$ & $\begin{array}{r}69.73 \\
1,274.00 \\
24,104.27\end{array}$ & $\begin{array}{r}13,260.00 \\
39,054.00 \\
131,258.00\end{array}$ & $\begin{array}{l}18.88 \\
37.16 \\
71.17\end{array}$ & $\begin{array}{r}145,414.00 \\
768,917.51 \\
3,138,143.00\end{array}$ & $\begin{array}{l}2.00 \\
4.85 \\
8.00\end{array}$ & \\
\hline
\end{tabular}

1 Not Reported. 2 Not charged to library budget. 3 1953-54 budget, actual expenditure not available. 4 Does not include summer salary. 5 Included in total books, periodicals and binding. 


\section{Teachers College Library Salary Statistics}

\begin{tabular}{|c|c|c|c|c|c|}
\hline & $\begin{array}{c}\text { Chief } \\
\text { Librarian }\end{array}$ & $\begin{array}{l}\text { Associate } \\
\text { Chief } \\
\text { Librarian }\end{array}$ & $\begin{array}{l}\text { Departm } \\
\text { Minimum }\end{array}$ & $\begin{array}{l}\text { Heads } \\
\text { Maximum }\end{array}$ & $\begin{array}{l}\text { Laboratory } \\
\text { School } \\
\text { Librarian }\end{array}$ \\
\hline 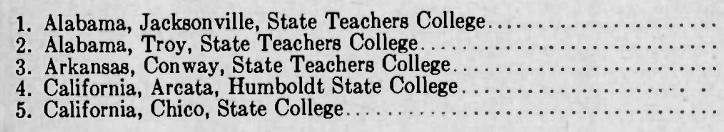 & $\begin{array}{r}\$ 4,100.00 \\
4,700.00 \\
7,728.00 \\
7,728.00\end{array}$ & $\begin{array}{l}3,400.00 \\
4,980.00 \\
\ldots \ldots \ldots 1\end{array}$ & $\begin{array}{l}\$ \ldots \ldots \\
\ldots \ldots \ldots \\
4,092.00\end{array}$ & $\begin{array}{r}\$ \ldots \ldots \ldots \\
\ldots \ldots \ldots \ldots \\
4,980.00 \\
5,496.00\end{array}$ & $\begin{array}{l}\$ \ldots \ldots \ldots \\
\ldots \ldots \ldots \\
4,092.00 \\
4,092.00\end{array}$ \\
\hline 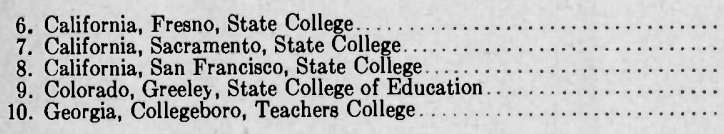 & $\begin{array}{l}7,728.00 \\
7,728.00 \\
7,728.00 \\
4,700.00 \\
5,300.00\end{array}$ & $\begin{array}{l}6,060.00 \\
6,060.00 \\
\ldots \ldots \ldots{ }^{1} \\
\ldots \ldots \ldots \cdots{ }^{1}\end{array}$ & $\begin{array}{l}4,512.00 \\
4,512.00 \\
4,512.00 \\
4,000.00\end{array}$ & $\begin{array}{l}5,496.00 \\
5,496.00 \\
6,060.00 \\
\dddot{1,200.00}\end{array}$ & $\begin{array}{l}4,092.00 \\
4,092.00 \\
4,533.00\end{array}$ \\
\hline 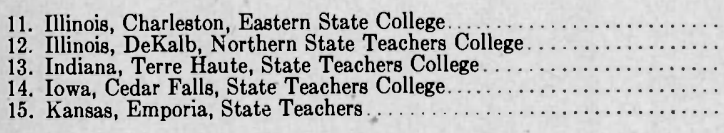 & $\begin{array}{l}8,910.00 \\
7,040.00 \\
6,600.00 \\
6,900.00 \\
\cdots\end{array}$ & $\begin{array}{l}6,270.00 \\
\cdots \cdots \cdots 0^{1} \\
\cdots \cdots \cdots\end{array}$ & $\begin{array}{l}5,700.00 \\
4,648.00^{10} \\
3,840.00 \\
4,400.00 \\
\cdots \cdots \cdots\end{array}$ & $\begin{array}{l}6,050.00 \\
4,920.00 \\
5,450.00 \\
\cdots \cdots \cdots\end{array}$ & $\begin{array}{l}5,500.00 \\
5,170.00 \\
4,565.00 \\
\cdots \cdots \cdots\end{array}$ \\
\hline 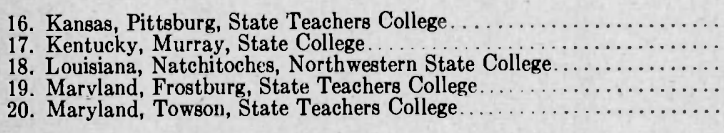 & $\begin{array}{l}6,000.00 \\
4,400.00 \\
7,875.00 \\
5,417.00 \\
5,417.00\end{array}$ & $\begin{array}{l}3,600.00 \\
6,250.00 \\
\cdots \cdots \cdots \cdots 1\end{array}$ & $\begin{array}{l}4,326.56 \\
\cdots \cdots \cdots 1_{1}^{1} \\
\cdots \cdots \cdots 1_{1}^{1} \\
\cdots \cdots \cdots \cdots 1\end{array}$ & $\begin{array}{l}4,827.70 \\
\cdots \cdots \cdots 1_{1}^{1} \\
\cdots \cdots \cdots \cdots \\
\cdots \cdots \cdots \cdots\end{array}$ & $3,568.33$ \\
\hline 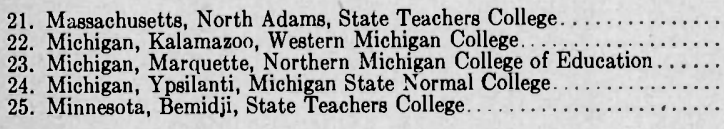 & $\begin{array}{l}5,760.00 \\
7,200.00 \\
5,975.00 \\
6,850.00 \\
5,460.00^{\circ}\end{array}$ & $\begin{array}{l}6,075.00 \\
\ldots \ldots \ldots \\
4,480.00\end{array}$ & $\begin{array}{l}5,650.00 \\
4,325.00 \\
\ldots \ldots \ldots\end{array}$ & $\begin{array}{l}5,800.00 \\
5650.00 \\
\ldots \ldots \ldots\end{array}$ & $\begin{array}{l}\ldots \ldots \ldots \\
\ldots, 600.00 \\
\ldots \ldots \ldots \ldots\end{array}$ \\
\hline 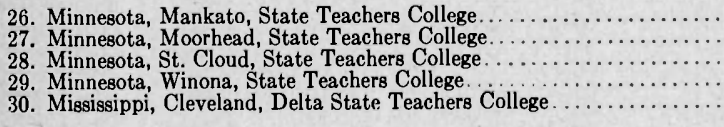 & $\begin{array}{l}5,160.00 \\
5,280.00 \\
5,720.00 \\
4,800.00^{4} \\
4,800.00\end{array}$ & $\begin{array}{l}5,280.00 \\
3,960.00^{4} \\
5,573.00 \\
4,680.00^{4} \\
\ldots \ldots \ldots\end{array}$ & $\begin{array}{l}4,980.00 \\
4,000.00\end{array}$ & $\begin{array}{l}\ldots \ldots \ldots \\
\cdots \ldots \ldots \\
\ddot{4}, 200.00\end{array}$ & $4,980.00$ \\
\hline 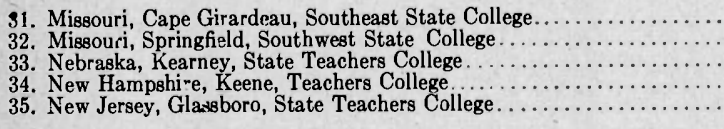 & $\begin{array}{l}5,820.00 \\
4,957.00 \\
5,000.00 \\
4,120.00 \\
6,180.00\end{array}$ & $\begin{array}{l}4,500.00 \\
4,957.00 \\
4,700.00 \\
3,940.00 \\
4,920.00\end{array}$ & $\begin{array}{l}3,200.00 \\
4,095.00 \\
\cdots \cdots \cdots \\
\cdots \cdots \cdots\end{array}$ & $\begin{array}{l}3,360.00 \\
4,405.00 \\
\cdots \cdots \cdots \\
\cdots \cdots \cdots\end{array}$ & $\begin{array}{l}\cdots \cdots \cdots \\
\cdots \cdots \cdots \\
\cdots \cdots \cdots \\
\cdots \cdots\end{array}$ \\
\hline 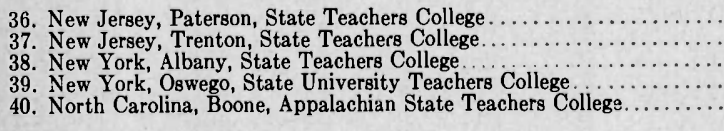 & $\begin{array}{l}7,140.00 \\
7,200.00 \\
6,906.00 \\
6,846.50 \\
5,396.00\end{array}$ & $\begin{array}{l}4,980.00 \\
5,448.00 \\
5,371.00 \\
\ldots \ldots \ldots\end{array}$ & $\begin{array}{l}\ldots \ldots \ldots \\
\ldots \ldots \ldots \\
3,100.00\end{array}$ & $\begin{array}{l}\cdots \cdots \\
\ldots \ldots \ldots \\
4,039.00\end{array}$ & $\begin{array}{l}\ldots \ldots \ldots \\
6,396.00 \\
4,052.00 \\
\ldots \ldots \ldots\end{array}$ \\
\hline 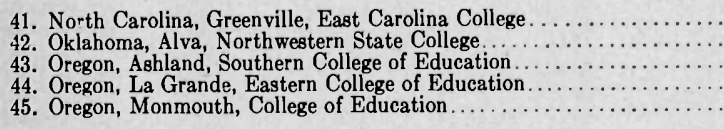 & $\begin{array}{l}7,419.00 \\
4,248.00^{4} \\
5,900.00 \\
5,800.00 \\
6,200.00\end{array}$ & $\begin{array}{l}4,248.00^{4} \\
\ldots \ldots \ldots \\
4,500.00\end{array}$ & $\begin{array}{l}4,000.00 \\
5,200.00 \\
4,800.00 \\
\cdots \cdots\end{array}$ & $\begin{array}{l}5,282.00 \\
5,900.00 \\
5,250.00 \\
\cdots \cdots\end{array}$ & $\begin{array}{l}4,752.00 \\
\cdots \cdots \cdots \\
\cdots \cdots \cdots \\
\cdots \cdots \cdots\end{array}$ \\
\hline 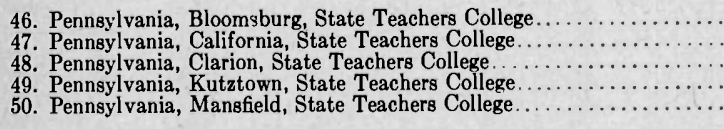 & $\begin{array}{l}5,500.00 \\
5,700.00 \\
5,260.00 \\
5,590.00^{4}\end{array}$ & $\begin{array}{l}5,260.00 \\
5,000.00 \\
4,950.00^{4}\end{array}$ & an. & $\cdots \cdots \cdots$ & $\begin{array}{l}5,370.00 \\
\cdots \ldots \ldots \\
4,522.00\end{array}$ \\
\hline 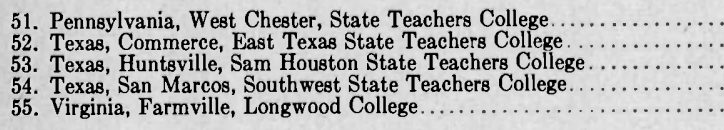 & $\begin{array}{l}6,913.00 \\
5,820.00 \\
6,000.00\end{array}$ & \begin{tabular}{l}
$5,590.00$ \\
$5,000.00$ \\
$\cdots \cdots \cdots$ \\
\hdashline, 320.00
\end{tabular} & $\begin{array}{l}3,300.00 \\
4,230.00 \\
3,800.00 \\
\cdots\end{array}$ & $\begin{array}{l}4,300.09 \\
4,780.03 \\
4,120.00 \\
\cdots \cdots \cdots\end{array}$ & 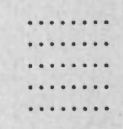 \\
\hline 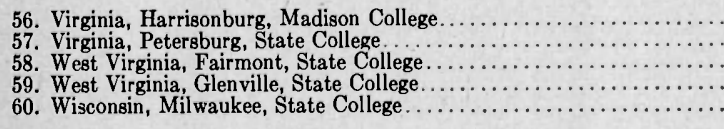 & $\begin{array}{l}5,200.00^{9} \\
7,680.00 \\
4,100.00^{4} \\
6,540.00\end{array}$ & $\begin{array}{l}\ldots \ldots \ldots \\
\ddot{4}, 800.00 \\
\ldots \ldots \ldots \\
\ldots \ldots \ldots\end{array}$ & $\begin{array}{l}3,540.00 \\
3,936.00 \\
3,600.00 \\
\dddot{4}, 215.00\end{array}$ & $\begin{array}{l}4,830.00 \\
4,920.00 \\
3,700.00 \\
5,390.00\end{array}$ & $\begin{array}{l}\cdots \cdots \cdots \\
\cdots \cdots \cdots \\
\cdots \cdots \cdots \\
\cdots \cdots \cdots\end{array}$ \\
\hline 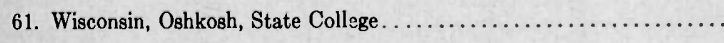 & $5,267.00$ & $4,367.00$ & n....... & $\cdots \cdots \cdots$ & $\cdots \cdots \cdots$ \\
\hline 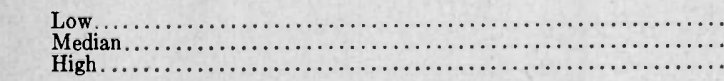 & $\begin{array}{r}4,100.00 \\
5,900.00 \\
8,910.00\end{array}$ & $\begin{array}{l}3,400.00 \\
4,975.00 \\
6,270.00\end{array}$ & $\begin{array}{l}3,100.00 \\
4,215.00 \\
5,700.00\end{array}$ & $\begin{array}{l}3,360.00 \\
4,980.00 \\
6,060.00\end{array}$ & $\begin{array}{l}3,568.33 \\
4,533.00 \\
6,396.00\end{array}$ \\
\hline
\end{tabular}




\section{3-1954 (as of September I, I954)}

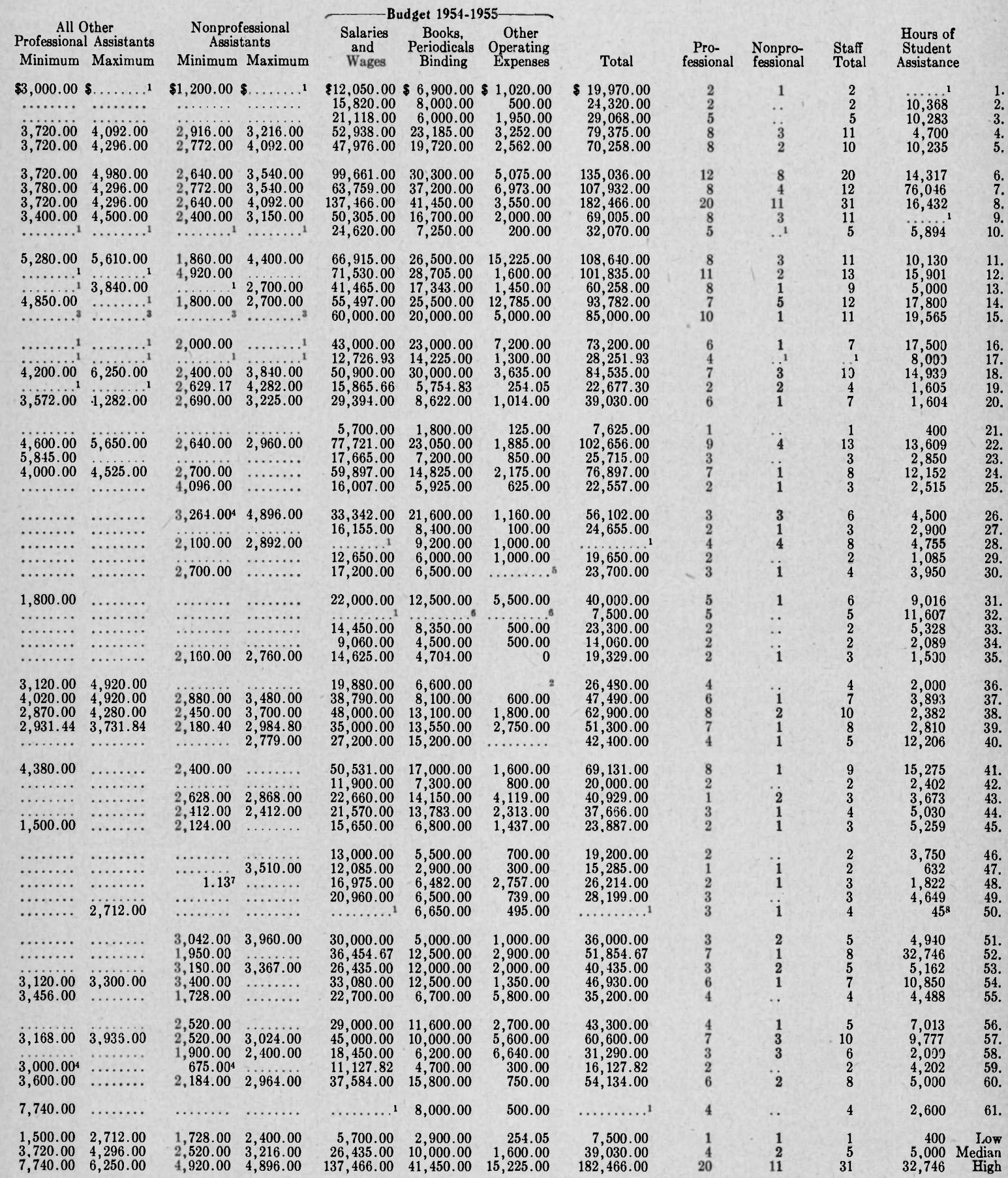

1 Not reported. 2 Not charged to library budget. 3 Confidential. 4 For 9 months. 5 Included in total books, pe:iodicals and binding. 6 Salary, wages, books, periodicals and binding not separate. 7 Hourly rate. 8 Hours per week, 9 For 10 months. 10 Mean for 8 staff members. 